\title{
Satisfiability Calculus: An Abstract Formulation of Semantic Proof Systems
}

\author{
Carlos G. Lopez Pombo* \\ CONICET-Universidad de Buenos Aires. \\ Instituto de Investigación en Ciencias de la \\ Computación (ICC) \\ clpombo@dc.uba.ar \\ Nazareno M. Aguirre \\ CONICET-Department of Computer Science \\ Universidad Nacional de Río Cuarto \\ Río Cuarto, Argentina \\ naguirre@dc.exa.unrc.edu.ar
}

\author{
Pablo F. Castro ${ }^{\dagger}$ \\ CONICET- Department of Computer Science \\ Universidad Nacional de Río Cuarto \\ Río Cuarto, Argentina \\ pcastro@dc.exa.unrc.edu.ar \\ Thomas S.E. Maibaum ${ }^{\S}$ \\ Department of Computing \& Software \\ McMaster University, Canada \\ tom@maibaum.org
}

\begin{abstract}
The theory of institutions, introduced by Goguen and Burstall in 1984, can be thought of as an abstract formulation of model theory. This theory has been shown to be particularly useful in computer science, as a mathematical foundation for formal approaches to software construction. Institution theory was extended by a number of researchers, José Meseguer among them, who, in 1989, presented General Logics, wherein the model theoretical view of institutions
\end{abstract}

Address for correspondence: Department of Computer Science, Facultad de Ciencias Exactas y Naturales, Universidad de Buenos Aires, Argentina

${ }^{*}$ Carlos G. Lopez Pombo wants to thank the founding from Agencia Nacional de Promoción Científica y Tecnológica (ANPCyT) through grant PICT 2013 No. 2129, from Universidad de Buenos Aires through grant UBACyT 2014-2017 No. 20020130200092BA and from Consejo Nacional de Investigaciones Científicas y Tecnológicas (CONICET) through grant PIP 2014-2016 No. 11220130100148CO.

${ }^{\dagger}$ Pablo Castro wants to thank the founding from Agencia Nacional de Promoción Científica y Tecnológica (ANPCyT) through grant PICT 2013 No. 0080.

${ }^{\ddagger}$ Nazareno Aguirre wants to thank the founding from Agencia Nacional de Promoción Científica y Tecnológica (ANPCyT) through grant PICT 2012 No. 1298.

${ }^{\S}$ Thomas S.E. Maibaum gratefully acknowledges the support of the National Science and Engineering Research Council of Canada and McMaster University. 
is complemented by providing (categorical) structures supporting the proof theory of any given logic. In other words, Meseguer introduced the notion of proof calculus as a formalisation of syntactical deduction, thus "implementing" the entailment relation of a given logic. In this paper we follow the approach initiated by Goguen and introduce the concept of Satisfiability Calculus. This concept can be regarded as the semantical counterpart of Meseguer's notion of proof calculus, as it provides the formal foundations for those proof systems that resort to model construction techniques to prove or disprove a given formula, thus "implementing" the satisfiability relation of an institution. These kinds of semantic proof methods have gained a great amount of interest in computer science over the years, as they provide the basic means for many automated theorem proving techniques.

Keywords: Institution Theory, Semantic Proof Systems, Formal Methods

\section{Introduction}

The theory of institutions [1] provides a formal and generic definition of the notion of logical system from an abstract model-theoretic point of view, mainly using constructions originating in category theory. In [2], Meseguer complemented the theory of institutions by providing a categorical characterization for the notions of entailment system (also called $\pi$-institutions by Fiadeiro and Sernadas in [3]) and the corresponding notion of proof calculi. Let us note that the original formulation of institutions stresses the semantic side of logical systems, leaving the deductive part implicit. This issue was noted in [4], where the authors proposed to encode the structure of proofs into the category of sentences of the corresponding institution; however, this idea was left as further work in that seminal paper.

Another important topic of research in this field is the characterisation of the possible functorial relations between different institutions, intuitively interpreted as semantics preserving translations between logical systems. In [4, 5, 6] Goguen and Burstall, Tarlecki, and Goguen and Roşu, respectively, define several kinds of these semantics preserving translations. The most obvious relation between institutions is the so-called institution morphism, introduced in [4]. Roughly speaking, an institution morphism captures a "structure forgetting" relationship between two logical systems. As shown in these articles, the collection of institutions together with the institution morphisms between them form the category Ins. Additionally, their dual morphisms (named institution co-morphisms [6] or institution representations [5]) also form a category denoted as Colns. Relations between institutions can be defined in many ways and their intuitive interpretation is obtained by examining the direction of the functorial relations between the components of the related institutions.

Several applications of the theory of institutions in computer science and software engineering have been proposed in the literature. Sannella and Tarlecki [7] studied how specifications in an arbitrary logical system can be structured by using the abstract setting provided by institutions, and later, in [8], the authors presented an abstract theory for software specification and development introducing the notion of refinement of specifications based on the idea of implementation step. In [9, 10] and $[11,12]$, we can find two alternative proposals for the use of institutions as a foundation for heterogeneous environments for software specification proposed by Mossakowski and Tarlecki, and Diaconescu, respectively. Institutions have also been proposed as a very general version of abstract 
model theory [13], offering a suitable formal framework for addressing heterogeneity in specifications $[14,15]$, including applications related to modelling languages such as UML [16] and other languages relevant in computer science and software engineering.

As we already mentioned, the original presentation of the theory of institutions provides an abstract definition of the notion of logical system, but only from its model-theoretical perspective. In this sense, deductive aspects of logical systems were relegated to satisfy the basic need of formalising theory presentations, and formulating soundness and completeness with respect to an ideal entailment relation. Extensions of the theory of institutions capturing proof-theoretical concepts have been extensively studied, most notably by Meseguer [2]. Roughly speaking, Meseguer proposed the extension of entailment systems with categorical concepts capturing the notion of proof in a very abstract and general way. In Meseguer's words:

A reasonable objection to the above definition of $\operatorname{logic}^{1}$ is that it abstracts away the structure of proofs, since we know only that a set $\Gamma$ of sentences entails another sentence $\varphi$, but no information is given about the internal structure of such a $\Gamma \vdash \varphi$ entailment. This observation, while entirely correct, may be a virtue rather than a defect, because the entailment relation is precisely what remains invariant under many equivalent proof calculi that can be used for a logic.

Thus, Meseguer's work concentrates on the introduction of proof-theoretical aspects of a logic, providing not only the definition of entailment system, but also complementing it with the notion of proof calculus, obtaining what Meseguer calls a logical system. Intuitively, the notion of proof calculus introduced by Meseguer provides an "implementation" of the entailment relation of a logic. Indeed, this approach corrected, in our view, the inherent imbalance in favour of models in institutions, enhancing syntactic aspects in the definition of logical systems.

It is interesting to note that the same lack of an operational view observed in the definition of entailment systems is still present in the case of the satisfaction relation of any given institution. That is, in the same way that an entailment system may be "implemented" in terms of different proof calculi, a satisfaction relation could be "implemented" in terms of different satisfiability checking procedures. Making these satisfiability checking procedures explicit in the characterization of logical systems is highly relevant, since many successful software analysis tools are based on them. For instance, many automated analysis tools rely on model construction, either for proving properties, as with model checkers, or for finding counterexamples, as with tableaux techniques or SAT-solving based tools. These techniques constitute an important stream of research in logic, in particular in relation to (semi)automated software validation and verification.

The so-called semantic proof systems can be traced back to the works of Beth [17, 18], Herbrand [19] and Gentzen [20]. Beth's ideas were used by Smullyan to formulate the tableaux method for firstorder predicate logic [21]. Herbrand's and Gentzen's works inspired the formulation of resolution systems presented by Robinson [22]. These methods have also been extended, for instance, to deal with temporal logics [23, 24], which are widely used in computer science and software engineering, for example, in program synthesis and requirements analysis [25, 26, 27]. Furthermore, note that methods

\footnotetext{
${ }^{1}$ Authors' note: Meseguer refers to a logic as a structure that is composed of an entailment system together with an institution, see Definition 2.7.
} 
like those based on resolution and tableaux are strongly related to the semantics of a logic, making them useful to guide the construction of models, a use not easily associated with pure deductive methods like natural deduction or Hilbert systems.

The main goal of this paper is to provide an abstract characterisation of this class of semanticsbased tools for logical systems. This is accomplished by introducing a categorical characterisation of the notion of satisfiability calculus, which embraces logical tools such as tableaux, resolution, Gentzen style sequents, and related formalisms. As mentioned above, this can be thought of as a semantic counterpart of Meseguer's formalisation of proof calculi. We explore the concept of mappings between satisfiability calculi. While the basic definition of satisfiability calculus, together with some examples, were introduced in [28], in this paper we provide detailed proofs for the theorems and show additional examples. Furthermore, we introduce the notions of satisfiability subcalculus and effective satisfiability subcalculus, and we present the category Sat (formed by satisfiability calculi and mappings between them) together with its theoretical properties (resp., Subsat formed by satisfiability subcalculi and mappings between them, and EffSubsat formed by effective satisfiability subcalculi and mappings between them).

The paper is organised as follows. In Section 2 we present the definitions and results we will use throughout this paper. In Section 3 we present a categorical formalisation of satisfiability calculus, satisfiability subcalculus and effective satisfiability calculus, and prove relevant results underpinning the definitions. We also present examples to illustrate the main ideas. In Section 4 we present two different types of mappings between satisfiability calculi, satisfiability subcalculi and effective satisfiability subcalculi, prove that satisfiability calculi (resp., satisfiability subcalculi and effective satisfiability subcalculi) form a category and study its structural properties. Finally, in Section 5, we draw some conclusions and describe further lines of research.

\section{Preliminaries}

From now on, we assume the reader has a nodding acquaintance with basic concepts from category theory [29, 30]. We mainly follow the notation introduced in [2].

An entailment system is defined by identifying a family of syntactic consequence relations indexed by the elements in the corresponding category of signatures. As usual, the entailment relations are required to satisfy reflexivity, monotonicity ${ }^{2}$ and transitivity. In addition, a notion of translation between signatures is considered.

\section{Definition 2.1. (Entailment system [2])}

An entailment system is a structure $\left\langle\right.$ Sign, Sen, $\left.\left\{\vdash^{\Sigma}\right\}_{\Sigma \in|\operatorname{Sign}|}\right\rangle$ satisfying the following conditions:

- Sign is a category of signatures,

- Sen : Sign $\rightarrow$ Set is a functor. Let $\Sigma \in|\operatorname{Sign}|$; then $\operatorname{Sen}(\Sigma)$ returns the set of $\Sigma$-sentences, and

\footnotetext{
${ }^{2}$ The theory of institutions and general logics focus on monotonic logics. The interested reader is referred to [31] for a presentation of entailment systems for default logic, a well-known non-monotonic logical system introduced by Reiter in [32] aiming at the formalisation of defeasible logical reasoning.
} 
- $\left\{\vdash^{\Sigma}\right\}_{\Sigma \in|\operatorname{Sign}|}$, where $\vdash^{\Sigma} \subseteq 2^{\operatorname{Sen}(\Sigma)} \times \operatorname{Sen}(\Sigma)$, is a family of binary relations such that for any $\Sigma, \Sigma^{\prime} \in|\operatorname{Sign}|,\{\phi\} \cup\left\{\phi_{i}\right\}_{i \in \mathcal{I}} \subseteq \operatorname{Sen}(\Sigma), \Gamma, \Gamma^{\prime} \subseteq \operatorname{Sen}(\Sigma)$, the following conditions are satisfied:

- reflexivity: $\{\phi\} \vdash^{\Sigma} \phi$,

- monotonicity: if $\Gamma \vdash^{\Sigma} \phi$ and $\Gamma \subseteq \Gamma^{\prime}$, then $\Gamma^{\prime} \vdash^{\Sigma} \phi$,

- transitivity: if $\Gamma \vdash^{\Sigma} \phi_{i}$ for all $i \in \mathcal{I}$ and $\left\{\phi_{i}\right\}_{i \in \mathcal{I}} \vdash^{\Sigma} \phi$, then $\Gamma \vdash^{\Sigma} \phi$, and

- $\vdash$-translation: if $\Gamma \vdash^{\Sigma} \phi$, then for any morphism $\sigma: \Sigma \rightarrow \Sigma^{\prime}$ in $\operatorname{Sign}, \operatorname{Sen}(\sigma)(\Gamma) \vdash^{\Sigma^{\prime}}$ $\operatorname{Sen}(\sigma)(\phi)$.

\section{Definition 2.2. (Theories [2])}

Let $\left\langle\right.$ Sign, Sen, $\left.\left\{\vdash^{\Sigma}\right\}_{\Sigma \in|\operatorname{Sign}|}\right\rangle$ be an entailment system. Its category of theories is $\mathrm{Th}=\langle\mathcal{O}, \mathcal{A}\rangle$ such that:

- $\mathcal{O}=\{\langle\Sigma, \Gamma\rangle|\Sigma \in| \operatorname{Sign} \mid$ and $\Gamma \subseteq \operatorname{Sen}(\Sigma)\}$, and

- $\mathcal{A}=\left\{\sigma:\langle\Sigma, \Gamma\rangle \rightarrow\left\langle\Sigma^{\prime}, \Gamma^{\prime}\right\rangle \mid\langle\Sigma, \Gamma\rangle,\left\langle\Sigma^{\prime}, \Gamma^{\prime}\right\rangle \in \mathcal{O}, \sigma: \Sigma \rightarrow \Sigma^{\prime} \in\|\operatorname{Sign}\|\right.$, for all $\left.\gamma \in \Gamma, \Gamma^{\prime} \vdash^{\Sigma^{\prime}} \operatorname{Sen}(\sigma)(\gamma)\right\}$.

In addition, if a morphism $\sigma:\langle\Sigma, \Gamma\rangle \rightarrow\left\langle\Sigma^{\prime}, \Gamma^{\prime}\right\rangle$ satisfies $\operatorname{Sen}(\sigma)(\Gamma) \subseteq \Gamma^{\prime}$, it is called axiom preserving. By retaining those morphisms of Th that are axiom preserving, we obtain the subcategory $\mathrm{Th}_{0}$.

Note that, in the previous definition, the objects of Th are defined to be a signature and a set of axioms, which are not necessarily closed under entailment. Other authors (c.f. [7], [33] or [13]) refer to such category as basic specifications, theory presentations or just presentations; then, the concept of theory is reserved for those objects satisfying to be closed under entailment. Even though this separation can be useful in many situations, we adopt the terminology introduced by Meseguer in [2] where no such difference is needed, and noticing that the present work can be understood as an extension of the categorical framework for defining logical systems presented in the aforementioned article.

\section{Definition 2.3. (Closure under entailment)}

Let $\left\langle\right.$ Sign, Sen, $\left.\left\{\vdash^{\Sigma}\right\}_{\Sigma \in|\operatorname{Sign}|}\right\rangle$ be an entailment system and $\langle\Sigma, \Gamma\rangle \in|\mathrm{Th}|$. We define ${ }^{\bullet}: 2^{\operatorname{Sen}(\Sigma)} \rightarrow$ $2^{\operatorname{Sen}(\Sigma)}$ as follows: $\Gamma^{\bullet}=\left\{\gamma \mid \Gamma \vdash^{\Sigma} \gamma\right\}$. This function is extended to elements of $T h$, by defining it as follows: $\langle\Sigma, \Gamma\rangle^{\bullet}=\left\langle\Sigma, \Gamma^{\bullet}\right\rangle$. $\Gamma^{\bullet}$ is called the theory generated by $\Gamma$.

\section{Definition 2.4. (Sensibility and simplicity [2])}

Let $\left\langle\right.$ Sign, Sen, $\left.\left\{\vdash^{\Sigma}\right\}_{\Sigma \in|\operatorname{Sign}|}\right\rangle$ and $\left\langle\operatorname{Sign}^{\prime}, \mathbf{S e n}^{\prime},\left\{\vdash^{{ }^{\Sigma}}\right\}_{\Sigma \in\left|\operatorname{Sign}^{\prime}\right|}\right\rangle$ be entailment systems, $\Phi: \mathrm{Th}_{0} \rightarrow$ $\mathrm{Th}^{\prime}{ }_{0}$ be a functor and $\alpha$ : Sen $\rightarrow$ Sen $^{\prime} \circ \Phi$ a natural transformation. $\Phi$ is said to be $\alpha$-sensible if the following conditions are satisfied:

1. there is a functor $\Phi^{\diamond}:$ Sign $\rightarrow \operatorname{Sign}^{\prime}$ such that $\operatorname{sign}^{\prime} \circ \Phi=\Phi^{\diamond} \circ$ sign, where sign : Th $\rightarrow$ Sign and $\operatorname{sign}^{\prime}: \mathrm{Th}^{\prime} \rightarrow \mathrm{Sign}^{\prime}$ are the forgetful functors from theory presentations to signatures, and 
2. if $\langle\Sigma, \Gamma\rangle \in|\mathrm{Th}|$ and $\left\langle\Sigma^{\prime}, \Gamma^{\prime}\right\rangle \in\left|\mathrm{Th}^{\prime}\right|$ such that $\Phi(\langle\Sigma, \Gamma\rangle)=\left\langle\Sigma^{\prime}, \Gamma^{\prime}\right\rangle$, then $\left(\Gamma^{\prime}\right)^{\bullet}=\left(\emptyset^{\prime} \cup\right.$ $\left.\alpha_{\Sigma}(\Gamma)\right)^{\bullet}$, where $\emptyset^{\prime}=\alpha_{\Sigma}(\emptyset)^{3}$.

$\Phi$ is said to be $\alpha$-simple if, instead of satisfying $\left(\Gamma^{\prime}\right)^{\bullet}=\left(\emptyset^{\prime} \cup \alpha_{\Sigma}(\Gamma)\right)^{\bullet}$ in Condition 2, the stronger condition $\Gamma^{\prime}=\emptyset^{\prime} \cup \alpha_{\Sigma}(\Gamma)$ is satisfied.

It is straightforward to see, based on the monotonicity of ${ }^{\bullet}$, that $\alpha$-simplicity implies $\alpha$-sensibility. An $\alpha$-sensible functor has the property that the associated natural transformation $\alpha$ depends only on signatures. This is a consequence of the following lemma.

Lemma 2.5. (Lemma 22, [2])

Let $\left\langle\right.$ Sign, Sen, $\left.\left\{\vdash^{\Sigma}\right\}_{\Sigma \in \mid \text { Sign } \mid}\right\rangle$ and $\left\langle\operatorname{Sign}^{\prime}\right.$, Sen $\left.^{\prime},\left\{\vdash^{\prime^{\Sigma}}\right\}_{\Sigma \in\left|\operatorname{Sign}^{\prime}\right|}\right\rangle$ be entailment systems and $\Phi: \mathrm{Th}_{0} \rightarrow$ $\mathrm{Th}_{0}^{\prime}$ a functor satisfying Condition 1 of Definition 2.4. Then any natural transformation $\alpha$ : Sen $\rightarrow$ $\mathbf{S e n}^{\prime} \circ \Phi$ can be obtained from a natural transformation $\alpha^{\diamond}: \operatorname{Sen}(\Sigma) \dot{\rightarrow} \operatorname{Sen}^{\prime} \circ \Phi^{\diamond}$ by horizontally composing with the functor sign : Th $\rightarrow$ Sign.

Roughly speaking, an institution is an abstract formalisation of the model theory of a logic in such a way that the existing relations between signatures, sentences over a signature and models for a signature are made explicit. These aspects are reflected by introducing the category of signatures, defining two functors capturing the sets of sentences and the classes of models, the first one going from this category to the category Set and the second one going from this category to Cat, and by requiring the satisfiability relation to remain invariant under signature change.

\section{Definition 2.6. (Institution [1])}

An institution is a structure of the form $\left\langle\right.$ Sign, Sen, Mod, $\left.\left\{\models^{\Sigma}\right\}_{\Sigma \in|\operatorname{Sign}|}\right\rangle$ satisfying the following conditions:

- Sign is a category of signatures,

- Sen : Sign $\rightarrow$ Set is a functor. Let $\Sigma \in|\operatorname{Sign}|$, then $\operatorname{Sen}(\Sigma)$ is its corresponding set of $\Sigma$-sentences,

- Mod : Sign ${ }^{\mathrm{op}} \rightarrow$ Cat is a functor. Let $\Sigma \in|\operatorname{Sign}|$, then $\operatorname{Mod}(\Sigma)$ is its corresponding category of $\Sigma$-models,

- $\left\{\mid=^{\Sigma}\right\}_{\Sigma \in|\operatorname{Sign}|}$ is a family of binary relations $\models^{\Sigma} \subseteq|\operatorname{Mod}(\Sigma)| \times \operatorname{Sen}(\Sigma)$, for all $\Sigma \in|\operatorname{Sign}|$

such that for all $\sigma: \Sigma \rightarrow \Sigma^{\prime} \in\|\operatorname{Sign}\|, \phi \in \operatorname{Sen}(\Sigma)$ and $\mathcal{M}^{\prime} \in\left|\operatorname{Mod}\left(\Sigma^{\prime}\right)\right|$, the following $=-$ invariance condition holds:

$$
\mathcal{M}^{\prime} \models{ }^{\Sigma^{\prime}} \operatorname{Sen}(\sigma)(\phi) \quad \text { iff } \quad \operatorname{Mod}\left(\sigma^{\text {op }}\right)\left(\mathcal{M}^{\prime}\right) \models^{\Sigma} \phi .
$$

Intuitively, the last condition above says that the notion of truth is invariant with respect to notation change. Given $\langle\Sigma, \Gamma\rangle \in|\mathrm{Th}|$, Mod : Th ${ }^{\mathrm{op}} \rightarrow$ Cat is the extension of the functor Mod : Sign ${ }^{\mathrm{op}} \rightarrow$ Cat such that $\operatorname{Mod}(\langle\Sigma, \Gamma\rangle)$ denotes the full subcategory of $\operatorname{Mod}(\Sigma)$ determined by those models

\footnotetext{
${ }^{3} \emptyset^{\prime}$ is not necessarily the empty set of axioms. This fact will be clarified later on.
} 
$\mathcal{M} \in|\operatorname{Mod}(\Sigma)|$ such that $\mathcal{M} \models{ }^{\Sigma} \gamma$, for all $\gamma \in \Gamma$. The relation $\models^{\Sigma}$ between sets of formulae and formulae is defined in the following way: given $\Sigma \in|\operatorname{Sign}|, \Gamma \subseteq \operatorname{Sen}(\Sigma)$ and $\alpha \in \operatorname{Sen}(\Sigma)$,

$$
\Gamma \models^{\Sigma} \alpha \quad \text { if and only if } \quad \mathcal{M} \models^{\Sigma} \alpha \text {, for all } \mathcal{M} \in|\operatorname{Mod}(\langle\Sigma, \Gamma\rangle)| .
$$

Now, from Definitions 2.1 and 2.6, it is possible to give a definition of logic by relating both its model-theoretic and proof-theoretic characterisations. In this respect, coherence between the semantic and syntactic entailment relations is required, reflecting the standard concepts of soundness and completeness of logical systems.

\section{Definition 2.7. (Logic [2])}

A logic is a structure $\left\langle\right.$ Sign, Sen, Mod, $\left.\left\{\left.\right|^{\Sigma}\right\}_{\Sigma \in|\operatorname{Sign}|},\left\{\mid \models^{\Sigma}\right\}_{\Sigma \in|\operatorname{Sign}|}\right\rangle$ satisfying the following conditions:

- $\left\langle\right.$ Sign, Sen, $\left.\left\{\vdash^{\Sigma}\right\}_{\Sigma \in|\operatorname{Sign}|}\right\rangle$ is an entailment system,

- $\left\langle\right.$ Sign, Sen, Mod, $\left.\left\{\mid=^{\Sigma}\right\}_{\Sigma \in \mid \text { Sign } \mid}\right\rangle$ is an institution, and

- the following soundness condition is satisfied: for any $\Sigma \in|\operatorname{Sign}|, \phi \in \operatorname{Sen}(\Sigma), \Gamma \subseteq \operatorname{Sen}(\Sigma)$ :

$$
\Gamma \vdash^{\Sigma} \phi \text { implies } \Gamma \models^{\Sigma} \phi \text {. }
$$

In addition, a logic is complete if the following condition is satisfied: for any $\Sigma \in|\operatorname{Sign}|, \phi \in \operatorname{Sen}(\Sigma)$, $\Gamma \subseteq \operatorname{Sen}(\Sigma):$

$$
\Gamma \models{ }^{\Sigma} \phi \quad \text { implies } \Gamma \vdash^{\Sigma} \phi .
$$

Definition 2.1 associates deductive relations to signatures. As already discussed, it is important to analyse how these relations are obtained. The next definition formalises the notion of proof calculus by associating a proof-theoretic structure to the deductive relations introduced by the definitions of entailment systems. As an example, in [2, Ex. 11, pp. 15], Meseguer presents natural deduction as one of the many proof calculi for first-order predicate logic by resorting to multicategories (see [2, Def. 10]).

\section{Definition 2.8. (Proof calculus [2])}

A proof calculus is a structure $\left\langle\operatorname{Sign}, \operatorname{Sen},\left\{\vdash^{\Sigma}\right\}_{\Sigma \in|\operatorname{Sign}|}, \mathbf{P}, \mathbf{P r}, \pi\right\rangle$ satisfying the following conditions:

- $\left\langle\right.$ Sign, Sen, $\left.\left\{\vdash^{\Sigma}\right\}_{\Sigma \in \mid \text { Sign } \mid}\right\rangle$ is an entailment system,

- $\mathbf{P}:$ Th $_{0} \rightarrow$ Struct $_{P C}$ is a functor. Let $T \in \mid$ Th $_{0} \mid$, then $\mathbf{P}(T) \in \mid$ Struct $_{P C} \mid$ is the prooftheoretical structure of $T^{4}$,

\footnotetext{
${ }^{4}$ The reader should note that Struct $P C$ strongly depends on the structure needed to formalise the concept of proof for a specific proof calculus. For example, while in [2, Ex. 11] the formalisation of natural deduction for first-order logic requires the use of multicategories [2, Def. 10], in [34, §3] the formalisation of the proof calculus for $\omega$-closure fork algebras with urelements [35, Def. 7] (a variant of fork algebras [36, 37] with a reflexive and transitive closure operator) requires the use of strict monoidal categories [29, Ch. VII, $\S 1]$ whose monoid of objects is given by the (not necessarily finite) subsets of the corresponding class of equations.
} 
- $\operatorname{Pr}:$ Struct $_{P C} \rightarrow$ Set is a functor. Let $T \in\left|T_{0}\right|$, then $\operatorname{Pr}(\mathbf{P}(T))$ is the set of proofs of $T$; the composite functor $\operatorname{Pr} \circ \mathbf{P}: \mathrm{Th}_{0} \rightarrow$ Set will be denoted by proofs, and

- $\pi$ : proofs $\rightarrow$ Sen is a natural transformation such that for each $T=\langle\Sigma, \Gamma\rangle \in\left|\mathrm{Th}_{0}\right|$ the image of $\pi_{T}: \operatorname{proofs}(T) \rightarrow \operatorname{Sen}(T)$ is the set $\Gamma^{\bullet}$. The map $\pi_{T}$ is called the projection from proofs to theorems for the theory $T$.

The use of the category $\mathrm{Th}_{0}$ for indexing proof structures responds to a technical need. Whenever we relate two theories with a morphism, say $\sigma:\langle\Sigma, \Gamma\rangle \rightarrow\left\langle\Sigma^{\prime}, \Gamma^{\prime}\right\rangle$, the previous definition imposes a need for extending that relation to proofs of the form $\pi: \emptyset \rightarrow \alpha \in|\operatorname{proofs}(\langle\Sigma, \Gamma\rangle)|$. If theories are taken from Th, we know that there exists $\pi^{\prime}: \emptyset \rightarrow \operatorname{Sen}(\sigma)(\alpha) \in\left|\operatorname{proofs}^{\prime}\left(\left\langle\Sigma^{\prime}, \Gamma^{\prime}\right\rangle\right)\right|$, but there is no obvious way to obtain it from $\pi$. If theories are taken from $\mathrm{Th}_{0}$, this problem no longer exists as the proof $\pi^{\prime}$ is obtained by applying exactly the same proof rules, obtaining the same proof structure (recall the inclusion $\operatorname{Sen}(\sigma)(\Gamma) \subseteq \Gamma^{\prime}$ ).

Finally, a logical system is defined as a logic plus a proof calculus for its proof theory.

\section{Definition 2.9. (Logical system [2])}

A structure $\left\langle\right.$ Sign, Sen, Mod, $\left.\left\{\vdash^{\Sigma}\right\}_{\Sigma \in \mid \text { Sign } \mid},\left\{\models^{\Sigma}\right\}_{\Sigma \in \mid \text { Sign } \mid}, \mathbf{P}, \mathbf{P r}, \pi\right\rangle$ is said to be a logical system if it satisfies the following conditions:

- $\left\langle\right.$ Sign, Sen, Mod, $\left.\left\{\vdash^{\Sigma}\right\}_{\Sigma \in|\operatorname{Sign}|},\left\{\models^{\Sigma}\right\}_{\Sigma \in|\operatorname{Sign}|}\right\rangle$ is a logic, and

- $\left\langle\right.$ Sign, Sen, $\left.\left\{\vdash^{\Sigma}\right\}_{\Sigma \in|\operatorname{Sign}|}, \mathbf{P}, \mathbf{P r}, \pi\right\rangle$ is a proof calculus.

\section{Satisfiability in General Logics}

In this section, we provide a definition of satisfiability calculus as the means for providing a corresponding operational formulation for the notion of satisfiability. A satisfiability calculus is the formal characterisation of a method for constructing models of a given theory, thus providing the semantic counterpart of a proof calculus. Roughly speaking, the semantic relation of satisfaction between a model and a formula can also be "implemented" by means of some kind of structure that depends on the model theory of the logic.

\section{Definition 3.1. (Satisfiability calculus)}

A structure $\left\langle\operatorname{Sign}\right.$, Sen, Mod, $\left\{\models^{\Sigma}\right\}_{\Sigma \in|\operatorname{Sign}|}, \mathbf{M}$, Mods, $\left.\mu\right\rangle$ is a satisfiability calculus if the following conditions are satisfied:

- $\left\langle\right.$ Sign, Sen, Mod, $\left.\left\{\mid=^{\Sigma}\right\}_{\Sigma \in|\operatorname{Sign}|}\right\rangle$ is an institution,

- $\mathbf{M}:$ Th $^{\text {op }} \rightarrow$ Struct $_{S C}$ is a functor. Let $T \in\left|\mathrm{Th}^{\mathrm{op}}\right|$, then $\mathbf{M}(T) \in \mid$ Struct $_{S C} \mid$ is the model structure of $T$,

- Mods : Struct $S C \rightarrow$ Cat is a functor. Let $T \in\left|\mathrm{Th}^{\mathrm{op}}\right|$, then $\operatorname{Mods}(\mathbf{M}(T))$ is a category of canonical (with respect to the structure formalised by Struct $_{S C}$ ) models of $T$; the composite functor Mods $\circ \mathrm{M}: \mathrm{Th}^{\mathrm{op}} \rightarrow$ Cat will be denoted by models, and 
- $\mu:$ models $\dot{\rightarrow} \mathscr{P} \circ$ Mod is a natural transformation such that, for each $T=\langle\Sigma, \Gamma\rangle \in\left|\mathrm{Th}_{0}{ }^{\mathrm{op}}\right|$, the image of $\mu_{T}: \operatorname{models}(T) \rightarrow \mathscr{P} \circ \operatorname{Mod}(T)$ is the (small) subcategory of $\operatorname{Mod}(T)$ corresponding to each canonical representation of a class of models in $|\operatorname{models}(T)|$. The map $\mu_{T}$ is called the projection of the category of models of the theory $T$.

The intuition behind the previous definition is as follows. For any theory $T$, the functor $\mathbf{M}$ assigns a structure in the category Struct $_{S C}$ representing the class of models for $T$. Notice that the target of functor $\mathbf{M}$, when applied to a theory $T$ is not necessarily a model but a structure representing the category of models of $T$. The reader may have already noticed that the functor $\mathbf{M}$ is contravariant with respect to category $T h$, reflecting the existing opposite direction of morphisms between categories of models with respect to those between signatures found in institutions (see Definition 2.6). The functor Mods maps the structure representing the class of models of a theory $T=\langle\Sigma, \Gamma\rangle$ to a category whose objects are canonical representations of models of $\Gamma$. Finally, for any theory $T$, the functor $\mu_{T}$ relates each of these structures to the corresponding subcategory of $\operatorname{Mod}(T)$, obtained via the composition of the functor Mod with the powerset functor $\mathscr{P}$.

\section{Example 3.2. (Tableau Method for First-Order Predicate Logic)}

Let us start by presenting the well-known tableaux method for first-order logic [21]. Let us denote by $\mathbb{I}_{F O L}=\left\langle\right.$ Sign, Sen, Mod, $\left.\left\{||^{\Sigma}\right\}_{\Sigma \in \mid \text { Sign } \mid}\right\rangle$ the institution of first-order predicate logic. Let $\Sigma \in \mid$ Sign $\mid$ and $S \subseteq \operatorname{Sen}(\Sigma)$; then a tableau for $S$ is a tree such that:

1. the nodes are labeled with sets of formulae (over $\Sigma$ ) and the root node is labeled with $S$,

2. if $u$ and $v$ are two connected nodes in the tree ( $u$ being an ancestor of $v$ ), then the label of $v$ is obtained from the label of $u$ by applying one of the following rules:

$$
\begin{gathered}
\frac{X \cup\{A \wedge B\}}{X \cup\{A \wedge B, A, B\}}[\wedge] \quad \frac{X \cup\{A \vee B\}}{X \cup\{A \vee B, A\} \quad X \cup\{A \vee B, B\}}[\vee] \\
\frac{X \cup\{\neg \neg A\}}{X \cup\{\neg \neg A, A\}}[\neg 1] \quad \frac{X \cup\{A\}}{X \cup\{A, \neg \neg A\}}[\neg 2] \quad \frac{X \cup\{A, \neg A\}}{\operatorname{Sen}(\Sigma)}[\text { false }] \\
\frac{X \cup\{\neg(A \wedge B)\}}{X \cup\{\neg(A \wedge B), \neg A \vee \neg B\}}\left[D M_{1}\right] \quad \frac{X \cup\{\neg(A \vee B)\}}{X \cup\{\neg(A \vee B), \neg A \wedge \neg B\}}\left[D M_{2}\right] \\
{[t \text { is a ground term. }] \frac{X \cup\{(\forall x) P(x)\}}{X \cup\{(\forall x) P(x), P(t)\}}[\forall] \quad[c \text { is a new constant. }] \frac{X \cup\{(\exists x) P(x)\}}{X \cup\{(\exists x) P(x), P(c)\}}[\exists]}
\end{gathered}
$$

A sequence of nodes $s_{0} \stackrel{\tau_{0}^{\alpha_{0}}}{\longrightarrow} s_{1} \stackrel{\tau_{1}^{\alpha_{1}}}{\longrightarrow} s_{2} \stackrel{\tau_{2}^{\alpha_{2}}}{\longrightarrow} \ldots$ is a branch if: $\left.a\right) s_{0}$ is the root node of the tree, and $b)$ for all $i \leq \omega, s_{i} \rightarrow s_{i+1}$ occurs in the tree, $\tau_{i}^{\alpha_{i}}$ is an instance of one of the rules presented above, and $\alpha_{i}$ are the formulae of $s_{i}$ to which the rule was applied. A branch $s_{0} \stackrel{\tau_{0}^{\alpha_{0}}}{\longrightarrow} s_{1} \stackrel{\tau_{1}^{\alpha_{1}}}{\longrightarrow} s_{2} \stackrel{\tau_{2}^{\alpha_{2}}}{\longrightarrow} \ldots$ in a tableau is saturated if there exists $i \leq \omega$ such that $s_{i}=s_{i+1}$. A branch $s_{0} \stackrel{\tau_{0}^{\alpha_{0}}}{\longrightarrow} s_{1} \stackrel{\tau_{1}^{\alpha_{1}}}{\longrightarrow} s_{2} \stackrel{\tau_{2}^{\alpha_{2}}}{\longrightarrow} \ldots$ in a tableau is closed if there exists $i \leq \omega$ and $\alpha \in \operatorname{Sen}(\Sigma)$ such that $\{\alpha, \neg \alpha\} \subseteq s_{i}$. 
Let $s_{0} \stackrel{\tau_{0}^{\alpha_{0}}}{\longrightarrow} s_{1} \stackrel{\tau_{1}^{\alpha_{1}}}{\longrightarrow} s_{2} \stackrel{\tau_{2}^{\alpha_{2}}}{\longrightarrow} \ldots$ be a branch in a tableau. Examining the rules presented above, it is straightforward to see that every $s_{i}$, with $i<\omega$, is a set of formulae. In each step, we have either the application of a rule decomposing one formula of the set into its constituent parts with respect to its major connective, while preserving satisfiability, or the application of the rule [false] denoting the fact that the corresponding set of formulae is unsatisfiable. Thus, the limit set of the branch is a set of formulae containing subformulae (and "instances" in the case of quantifiers) of the original set of formulae for which the tableau was built. As a result of this, every open branch represents, by means of the set of formulae occurring in the leaf, the class of models satisfying them.

In order to define the tableau method as a satisfiability calculus, we have to provide formal definitions for the categories supporting tableaux structures, for the functors $\mathbf{M}$ and Mods and for the natural transformation $\mu$. First, given $\Sigma \in|\operatorname{Sign}|$ and $\Gamma \subseteq \operatorname{Sen}(\Sigma)$, we define $\operatorname{Str}^{\Sigma, \Gamma}=\langle\mathcal{O}, \mathcal{A}\rangle$ such that $\mathcal{O}=2^{\operatorname{Sen}(\Sigma)}$ and $\mathcal{A}=\left\{\alpha:\left\{A_{i}\right\}_{i \in \mathcal{I}} \rightarrow\left\{B_{j}\right\}_{j \in \mathcal{J}} \mid \alpha=\left\{\alpha_{j}\right\}_{j \in \mathcal{J}}\right\}$, where for all $j \in \mathcal{J}$, $\alpha_{j}$ is a branch in a tableau for $\Gamma \cup\left\{B_{j}\right\}$ with leaves $\Delta \subseteq\left\{A_{i}\right\}_{i \in \mathcal{I}} S t r^{\Sigma, \Gamma}$ can be proved to be a category (see Lemma A.2 for a detailed proof). Then, we can prove that $\left\langle\operatorname{Str}^{\Sigma, \Gamma}, \cup, \emptyset\right\rangle$, where $\cup: \operatorname{Str}^{\Sigma, \Gamma} \times \operatorname{Str}^{\Sigma, \Gamma} \rightarrow \operatorname{Str}^{\Sigma, \Gamma}$ is the typical bi-functor on sets and functions, and $\emptyset$ is the neutral element for $\cup$, is a strict monoidal category (see Lemma A.3 for details).

Second, using the previous definition we can introduce the class of legal tableaux (denoted by Struct $_{S C}$ ), together with a class of arrows, and prove it is a category. Struct $S_{S C}$ is defined as $\langle\mathcal{O}, \mathcal{A}\rangle^{\text {op }}$ where $\mathcal{O}=\left\{\left\langle\operatorname{Str}^{\Sigma, \Gamma}, \cup, \emptyset\right\rangle|\Sigma \in| \operatorname{Sign} \mid \wedge \Gamma \subseteq \operatorname{Sen}(\Sigma)\right\}$, and $\mathcal{A}=\left\{\widehat{\sigma}:\left\langle\operatorname{Str}^{\Sigma, \Gamma}, \cup, \emptyset\right\rangle \rightarrow\right.$ $\left.\left\langle\operatorname{Str}^{\Sigma^{\prime}, \Gamma^{\prime}}, \cup, \emptyset\right\rangle \mid \sigma:\langle\Sigma, \Gamma\rangle \rightarrow\left\langle\Sigma^{\prime}, \Gamma^{\prime}\right\rangle \in\|\mathrm{Th}\|\right\}$, the homomorphic extensions of the morphisms in $\|\mathrm{Th}\|$ to sets of formulae preserving the application of rules (i.e., the structure of the tableaux) (see Lemma A.5 for a detailed proof).

Third, the functor $\mathbf{M}$ must be understood as the relation between a theory in $|\mathrm{Th}|$ and its corresponding category of structures representing legal tableaux. So, for every theory $\langle\Sigma, \Gamma\rangle, \mathbf{M}$ associates to it the strict monoidal category [29, Sec. 1, pp. 157] $\left\langle\operatorname{Str}^{\Sigma, \Gamma}, \cup, \emptyset\right\rangle$, and for every theory morphism $\sigma:\langle\Sigma, \Gamma\rangle \rightarrow\left\langle\Sigma^{\prime}, \Gamma^{\prime}\right\rangle \in\|\mathrm{Th}\|$ observed in the opposite direction, $\mathbf{M}$ associates to it a morphism $\widehat{\sigma}: S t r^{\Sigma, \Gamma} \rightarrow S t r^{\Sigma^{\prime}, \Gamma^{\prime}}$ which is the homomorphic extension of $\sigma$ to the structure of the tableaux, also observed in the opposite direction. Then, $\mathbf{M}:$ Th $^{\text {op }} \rightarrow$ Struct $_{S C}$ is defined as $\mathbf{M}(\langle\Sigma, \Gamma\rangle)=\left\langle S \operatorname{tr}^{\Sigma, \Gamma}, \cup, \emptyset\right\rangle$ and for any $\sigma:\langle\Sigma, \Gamma\rangle \rightarrow\left\langle\Sigma^{\prime}, \Gamma^{\prime}\right\rangle \in\|\mathrm{Th}\|, \mathbf{M}\left(\sigma^{\mathrm{op}}\right)=\widehat{\sigma}^{\mathrm{op}}$, where $\widehat{\sigma}:\left\langle\operatorname{Str}^{\Sigma, \Gamma}, \cup, \emptyset\right\rangle \rightarrow\left\langle\operatorname{Str}^{\Sigma^{\prime}, \Gamma^{\prime}}, \cup, \emptyset\right\rangle$ is the homomorphic extension of $\sigma$ to the structures in $\left\langle\operatorname{Str}^{\Sigma, \Gamma}, \cup, \emptyset\right\rangle$ (see Lemma A.7 for a detailed proof).

Fourth, the functor Mods provides the means for obtaining theory presentations characterising classes of models from structures of the form $S \operatorname{tr}^{\Sigma, \Gamma}$ by identifying the sets of formulae in the leaves of the open branches of a tableau. To this effect, Mods : Struct $S C \rightarrow$ Cat is defined on objects as $\operatorname{Mods}\left(\left\langle S t r^{\Sigma, \Gamma}, \cup, \emptyset\right\rangle\right)=\langle\mathcal{O}, \mathcal{A}\rangle$ where:

$$
\begin{gathered}
\mathcal{O}=\bigcup_{\langle\Sigma, \Delta\rangle \in|\mathrm{Th}|}\left\{\langle\Sigma, \widetilde{\Delta}\rangle \in|\mathrm{Th}| \mid\left(\exists \alpha: \Delta \rightarrow \emptyset \in\left\|\operatorname{Str}^{\Sigma, \Gamma}\right\|\right)\left(\forall \alpha^{\prime}: \Delta^{\prime} \rightarrow \Delta \in\left\|\operatorname{Str}^{\Sigma, \Gamma}\right\|\right),\right. \\
\left.\left(\Delta^{\prime}=\Delta\right) \wedge(\widetilde{\Delta} \rightarrow \emptyset \in \alpha) \wedge \neg(\exists \varphi)(\{\neg \varphi, \varphi\} \subseteq \widetilde{\Delta})\right\}
\end{gathered},
$$

(i.e., 1. the existentially quantified $\alpha: \Delta \rightarrow \emptyset \in\left\|S t r^{\Sigma, \Gamma}\right\|$ is a tableau, 2. stating that for any $\alpha^{\prime}: \Delta^{\prime} \rightarrow \Delta \in\left\|\operatorname{Str}^{\Sigma, \Gamma}\right\|$, the equation $\Delta^{\prime}=\Delta$ holds, expresses that $\alpha$ is saturated, and 3. requesting that $\widetilde{\Delta}$, where $\widetilde{\Delta} \rightarrow \emptyset$ is a branch of $\alpha$, is not inconsistent, expresses that it is the set of formulae at the leaf of an open branch), and 
- $\mathcal{A}=\left\{i d_{T}: T \rightarrow T \mid T \in \mathcal{O}\right\}$, (i.e., only the identities);

and on morphisms as for all $\sigma:\langle\Sigma, \Gamma\rangle \rightarrow\left\langle\Sigma^{\prime}, \Gamma^{\prime}\right\rangle \in\|\operatorname{Th}\|, \operatorname{Mods}\left(\widehat{\sigma}^{\mathrm{op}}\right)(\langle\Sigma, \delta\rangle)=\left\langle\Sigma^{\prime}, \operatorname{Sen}(\sigma)(\delta)\right\rangle$. This is proved to be a functor (see Lemma A.9 for a detailed proof).

Finally, $\mu$ has to relate the structures representing saturated tableaux with the model satisfying the set of formulae denoted by the source of the morphism; then we can define $\mu_{\langle\Sigma, \Delta\rangle}$ : $\operatorname{models}(\langle\Sigma, \Delta\rangle) \rightarrow \mathscr{P} \circ \operatorname{Mod}_{F O L}(\langle\Sigma, \Delta\rangle)$ as for all $\langle\Sigma, \delta\rangle \in|\operatorname{model} \mathbf{s}(\langle\Sigma, \Delta\rangle)|, \mu_{\langle\Sigma, \Delta\rangle}(\langle\Sigma, \delta\rangle)=$ $\operatorname{Mod}_{F O L}(\langle\Sigma, \delta\rangle)$ and prove it to be a natural transformation (see Lemma A.12 for details).

From all this, we can conclude that $\left\langle\right.$ Sign, Sen, Mod, $\left\{\mid=^{\Sigma}\right\}_{\Sigma \in|\operatorname{Sign}|}, \mathbf{M}$, Mods, $\left.\mu\right\rangle$ is a satisfiability calculus. The reader interested in the details of the proofs is pointed to Section A.1 of Appendix A.

Another important kind of system used by automated theorem provers are the so-called resolution methods. Below, we show how any resolution system conforms to the definition of satisfiability calculus.

\section{Example 3.3. (Resolution Method for First-Order Predicate Logic)}

As a second example we introduce resolution for first-order logic as described in [38]. We use the following notation: [ ] denotes the empty list; $[A]$ denotes the unitary list containing the formula $A$; $\ell_{0}, \ell_{1}, \ldots$ are variables ranging over lists; and $\ell_{i}+\ell_{j}$ denotes the concatenation of lists $\ell_{i}$ and $\ell_{j}$. Resolution builds a list of lists representing a disjunction of conjunctions. The rules for resolution are the following:

$$
\begin{aligned}
& \frac{\ell_{0}+[\neg \neg A]+\ell_{1}}{\ell_{0}+[A]+\ell_{1}}[\neg \neg]
\end{aligned}
$$

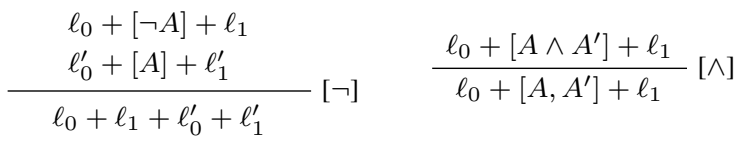

$$
\begin{aligned}
& \frac{\ell_{0}+\left[\neg\left(A \vee A^{\prime}\right)\right]+\ell_{1}}{\ell_{0}+\left[\neg A, \neg A^{\prime}\right]+\ell_{1}}[\neg \vee] \\
& \begin{array}{c}
\frac{\ell_{0}+\left[A \vee A^{\prime}\right]+\ell_{1}}{\ell_{0}+[A]+\ell_{1}}[\vee] \\
\ell_{0}+\left[A^{\prime}\right]+\ell_{1}
\end{array} \\
& \frac{\ell_{0}+\left[\neg\left(A \wedge A^{\prime}\right)\right]+\ell_{1}}{\ell_{0}+[\neg A]+\ell_{1}}[\neg \wedge] \\
& \ell_{0}+\left[\neg A^{\prime}\right]+\ell_{1} \\
& \text { for any closed term } t \frac{\ell_{0}+[\forall x: A(x)]+\ell_{1}}{\ell_{0}+[A[x / t]]+\ell_{1}}[\forall] \\
& \text { for a new constant } c \frac{\ell_{0}+[\exists x: A(x)]+\ell_{1}}{\ell_{0}+[A[x / c]]+\ell_{1}}[\exists]
\end{aligned}
$$

where $A(x)$ denotes a formula with free variable $x$, and $A[x / t]$ denotes the formula resulting from replacing variable $x$ by term $t$ everywhere in $A$. For the sake of simplicity, we assume that lists of formulae do not contain repeated elements. A resolution is a sequence of lists of formulae. If a resolution contains an empty list, we say that the resolution is closed; otherwise it is an open resolution.

For every signature $\Sigma \in|\operatorname{Sign}|$ and each $\Gamma \subset \operatorname{Sen}(\Sigma)$, we denote by $\operatorname{Str}^{\Sigma, \Gamma}$ the category whose objects are lists of formulae, and where every morphism $\sigma:\left[A_{0}, \ldots, A_{n}\right] \rightarrow\left[A_{0}^{\prime}, \ldots, A_{m}^{\prime}\right]$ represents a sequence of applications of resolution rules to $\left[A_{0}^{\prime}, \ldots, A_{m}^{\prime}\right]$ leading to $\left[A_{0}, \ldots, A_{n}\right]$. Then, Struct $_{S C}$ is a category whose objects are $\operatorname{Str}^{\Sigma, \Gamma}$, for each signature $\Sigma \in|\operatorname{Sign}|$ and set of formulae $\Gamma \in \operatorname{Sen}(\Sigma)$, and whose morphisms are of the form $\widehat{\sigma}: \operatorname{Str}^{\Sigma, \Gamma} \rightarrow \operatorname{Str}^{\Sigma^{\prime}, \Gamma^{\prime}}$, obtained by homomorphically extending $\sigma:\langle\Sigma, \Gamma\rangle \rightarrow\left\langle\Sigma^{\prime}, \Gamma^{\prime}\right\rangle$ in $\|\mathrm{Th}\|$.

As for the case of Example 3.2, the functor $\mathbf{M}:$ Th$^{\text {op }} \rightarrow$ Struct $_{S C}$ is defined as $\mathbf{M}(\langle\Sigma, \Gamma\rangle)=$ $\left\langle S t r^{\Sigma, \Gamma}, \cup, \emptyset\right\rangle$, and Mods : Struct $S C \rightarrow$ Cat is defined as in the previous example. Adapting the proofs given for Example 3.2 to this example is straightforward. 
A typical use for the methods described above is the search for counterexamples of a given logical property (i.e., as a refutation procedure). For instance, if we are interested in knowing if a certain property $\alpha$ follows from a set of formulae $\Gamma$, one can try to refute $\alpha$ by building a model for $\Gamma \cup\{\neg \alpha\}$ resorting to a tableaux method. In this way, one starts by applying rules to $\Gamma \cup\{\neg \alpha\}$, and once a saturated tableau is reached, if all the branches are closed, then there is no model of $\Gamma$ also satisfying $\neg \alpha$, indicating that $\alpha$ follows from $\Gamma$. On the other hand, if there exists an open branch, the limit set of that branch characterises a class of models satisfying both $\Gamma$ and $\neg \alpha$, exhibiting a counterexample for the formula of interest. Note the contrast with Hilbert systems, where one starts from the axioms by applying deduction rules until the desired formula is obtained.

As we mentioned in Section 1, Meseguer not only developed the idea of formalising the notion of proof calculus as an "implementation" of the entailment relation of an entailment system, but he also explored the possibility of restricting a proof calculus producing a subcalculus. The aim of this definition is to formally characterise the existence of specialised proof calculi, enjoying specific properties, for specific fragments of the entailment relation. The next definition presents a restriction of satisfiability calculus in a way analogous to that given by Meseguer in [2].

\section{Definition 3.4. (Satisfiability subcalculus)}

A structure of the form 〈Sign, Sen, Mod, $\left.\operatorname{Sign}_{0}, \mathbf{a x},\left\{\models^{\Sigma}\right\}_{\Sigma \in|\operatorname{Sign}|}, \mathbf{M}, \mathbf{M o d s}, \mu\right\rangle$ is satisfiability subcalculus if it satisfies the following conditions:

- $\left\langle\right.$ Sign, Sen, Mod, $\left.\left\{\mid=^{\Sigma}\right\}_{\Sigma \in|\operatorname{Sign}|}\right\rangle$ is an institution,

- $\operatorname{Sign}_{0}$ is a subcategory of Sign called the subcategory of admissible signatures; the restriction of the functor Sen to Sign $n_{0}$ will be denoted by $\operatorname{Sen}_{0}$,

- ax $: \operatorname{Sign}_{0} \rightarrow$ Set is a subfunctor of the functor obtained by composing $\mathbf{S e n}_{0}$ with the powerset functor, i.e., there is a natural inclusion $\operatorname{ax}(\Sigma) \subseteq \mathscr{P}\left(\operatorname{Sen}_{0}(\Sigma)\right)$ for each $\Sigma \in \operatorname{Sign}_{0}$. Each $\Gamma \in \operatorname{ax}(\Sigma)$ is called a set of admissible axioms. This defines a subcategory $\mathrm{Th}_{a x}$ of $\mathrm{Th}$ whose objects are theory presentations $T=\langle\Sigma, \Gamma\rangle$ with $\Sigma \in \operatorname{Sign}_{0}$ and $\Gamma \in \operatorname{ax}(\Sigma)$, and whose morphisms are axiom-preserving theory morphisms $H$ such that $H$ is in $\operatorname{Sign}_{0}$.

- $\mathbf{M}: \mathrm{Th}_{a x}{ }^{\mathrm{op}} \rightarrow$ Struct $_{S C}$ is a functor. Let $T \in\left|\mathrm{Th}_{a x}\right|$, then $\mathbf{M}(T) \in \mid$ Struct $_{S C} \mid$ is the model structure of $T$,

- Mods : Struct $S C \rightarrow$ Cat is a functor. Let $T \in\left|\mathrm{Th}_{a x}\right|$, then $\operatorname{Mods}(\mathbf{M}(T))$ is the set of canonical models of $T$; the composite functor Mods $\circ \mathbf{M}: \mathrm{Th}_{a x}{ }^{\mathrm{op}} \rightarrow$ Cat will be denoted by models, and

- $\mu:$ models $\dot{\rightarrow} \mathscr{P} \circ$ Mod is a natural transformation such that, for each $T=\langle\Sigma, \Gamma\rangle \in\left|\mathrm{Th}_{0}{ }^{\mathrm{op}}\right|$, the image of $\mu_{T}: \operatorname{models}(T) \rightarrow \mathscr{P} \circ \operatorname{Mod}(T)$ is the subcategory of $\operatorname{Mod}(T)$ corresponding to each canonical representation of a class of models in $|\operatorname{model} s(T)|$. The map $\mu_{T}$ is called the projection of the category of models of the theory $T$. 
Note that, in the definition above, the components M, Mods and $\mu$ may change depending on ax, thus we cannot define the notion of satisfiability subcalculus in terms of that of satisfiability calculus (similarly for Definition 3.5 below).

There are no major differences with respect to [2, Def. 14], except for the lack of restrictions on the possible conclusions that can be drawn with the subcalculus. Note that, in the case of prooftheoretic approaches, the introduction of a functor restricting the conclusions as a subfunctor of Sen is a key element of the definition, in contrast to satisfiability calculi where models being constructed are not related to any particular formula besides the set of formulae the model has to satisfy. The main addition in the definition of a satisfiability subcalculus with respect to a satisfiability calculus is the appearance of the functor ax restricting the sets of formulae to which the satisfiability subcalculus can be applied.

The following example illustrates Definition 3.4 by restricting a satisfiability calculus for firstorder modal logic to its term-free fragment.

\section{Example 3.5. (Sat. Subcalculus for the Term-Free Fragment of First-Order Modal Logic)}

Clarence Irving Lewis established the foundations of modal logics in 1910, in his PhD thesis [39], and subsequent research material culminating in [40], where he introduced five logical systems. These systems (named S1 to S5) assume different interpretations of modalities. First and second order extensions of Lewis' S2, S4, and S5, together with their corresponding axiomatic systems, were later developed by Barcan in $[41,42,43]$. The present era of modal logics started with the introduction of relational semantics, commonly referred to as "possible worlds" semantics, by Saul Kripke in [44, 45].

We restrict the satisfiability calculus (based on tableaux) for first-order modal logic, presented by Fitting in [46], to its term-free fragment. The presentation follows [47] with the sole exception of the feature that, as we did in Example 3.2, nodes in the tableaux will be sets of formulae rather than just singleton sets. As usual, when dealing with modal logics, we may assume a rigid or a flexible interpretation of the domains. For the sake of simplicity, we will assume the rigid setting.

Let $\mathbb{I}_{F O M L}=\left\langle\operatorname{Sign}\right.$, Sen, Mod, $\left.\left\{\left.\right|^{\Sigma}\right\}_{\Sigma \in|\operatorname{Sign}|}\right\rangle$ be the institution of first-order modal logic defined as follows:

1. Sign is the category of first-order signatures whose objects are of shape $\langle\mathcal{C}, \mathcal{F}, \mathcal{P}\rangle$, where $\mathcal{C}, \mathcal{F}$ and $\mathcal{P}$ are denumerable sets of constant, function and predicate symbols, respectively. Function and predicate symbols have an associated arity. The morphisms in Sign are 3-tuples of total mappings between the corresponding sets of symbols, with the second and third components, being arity-preserving.

2. Sen is a functor defined as follows: given $\Sigma=\langle\mathcal{C}, \mathcal{F}, \mathcal{P}\rangle \in|\operatorname{Sign}|$ and $\mathcal{X}$ a set of variable symbols, $\operatorname{Sen}(\Sigma)$ is the subset of the smallest set $\operatorname{Form}(\Sigma, \mathcal{X})$ of formulae obtained by the following grammar:

$$
\begin{aligned}
\operatorname{Term}(\Sigma, \mathcal{X})::= & c, c \in \mathcal{C}|x, x \in \mathcal{X}| f\left(t_{1}, \ldots, t_{\text {arity }(f)}\right), f \in \mathcal{F},\left\{t_{1}, \ldots, t_{\text {arity }(f)}\right\} \subseteq \operatorname{Term}(\Sigma, \mathcal{X}) \\
\operatorname{Form}(\Sigma, \mathcal{X})::= & P\left(t_{1}, \ldots, t_{\text {arity }(P)}\right), P \in \mathcal{P},\left\{t_{1}, \ldots, t_{\text {arity }(P)}\right\} \subseteq \operatorname{Term}(\Sigma, \mathcal{X})|\neg \operatorname{Form}(\Sigma, \mathcal{X})| \\
& \operatorname{Form}(\Sigma, \mathcal{X}) \vee \operatorname{Form}(\Sigma, \mathcal{X})|(\exists x) \operatorname{Form}(\Sigma, \mathcal{X}), x \in \mathcal{X}| \diamond \operatorname{Form}(\Sigma, \mathcal{X})
\end{aligned}
$$

containing only all the sentences (i.e., formulae with no free variables). Given a morphism $\sigma: \Sigma \rightarrow \Sigma^{\prime} \in\|\operatorname{Sign}\|$, the function between the corresponding sets of sentences obtained 
as $\operatorname{Sen}(\sigma)$ is the homomorphic extension of $\sigma$ to the structure of the fomulae as shown in the previous grammar.

3. Mod is a functor defined as follows: given $\Sigma=\langle\mathcal{C}, \mathcal{F}, \mathcal{P}\rangle \in|\operatorname{Sign}|$ and $\mathcal{X}$ a set of variable symbols, $\operatorname{Mod}(\Sigma)$ is the category whose objects are structures consisting of: a) an augmented frame [47, Def. 4.6.1] $\langle\Delta, \mathcal{R}, S\rangle$ where $\Delta$ is a set of worlds, $\mathcal{R} \subseteq \Delta \times \Delta$ and $S$ a domain; and b) a function $\mathcal{L}$ such that for all $w \in \Delta, \mathcal{L}(w)$ is a first-order interpretation of the nonlogical symbols in $\Sigma$ of shape $\mathcal{I}=\left\langle S,\left\{c^{\mathcal{I}}\right\}_{c \in \mathcal{C}},\left\{f^{\mathcal{I}}\right\}_{f \in \mathcal{F}},\left\{P^{\mathcal{I}}\right\}_{P \in \mathcal{P}}\right\rangle$ (see for instance [48]); and morphisms are homomorphisms between these structures. Finally, given $\sigma: \Sigma \rightarrow \Sigma^{\prime} \in$ $\|\operatorname{Sign}\|, \operatorname{Mod}(\sigma)$ denotes the usual (contravariant) reduct operation between the corresponding categories of models.

4. Given $\Sigma \in|\operatorname{Sign}|$, the satisfaction relation $\left.\right|_{\Sigma} \subseteq|\operatorname{Mod}(\Sigma)| \times|\operatorname{Sen}(\Sigma)|$ is defined in terms of the internal satisfaction relation as follows: for all $\langle\Delta, \mathcal{R}, S, \mathcal{L}\rangle \in|\operatorname{Mod}(\Sigma)|, \alpha \in \operatorname{Sen}(\Sigma)$

$$
\langle\Delta, \mathcal{R}, S, \mathcal{L}\rangle \models_{\Sigma} \alpha \text { iff for all } w \in \Delta \text { and } v: \mathcal{X} \rightarrow S,\langle\Delta, \mathcal{R}, S, \mathcal{L}\rangle, w, v \models_{\Sigma} \alpha
$$

Internal satisfaction is then defined as follows: let $\mathcal{M}=\langle\Delta, \mathcal{R}, S, \mathcal{L}\rangle$ be a model for signature $\langle\mathcal{C}, \mathcal{F}, \mathcal{P}\rangle, w \in \Delta$ and $v: \mathcal{X} \rightarrow S$ a valuation:

$$
\begin{array}{rlc}
\mathcal{M}, w, v \models_{\Sigma} P\left(t_{1}, \ldots, t_{\text {arity }(P)}\right) & \text { iff } & \left(m_{w, v}^{\mathcal{M}}\left(t_{1}\right), \ldots, m_{w, v}^{\mathcal{M}}\left(t_{\text {arity }(P)}\right)\right) \in P \mathcal{L}(w), \text { for all } P \in \mathcal{P}, \\
\mathcal{M}, w, v \models_{\Sigma} \neg \alpha & \text { iff } & \text { it does not hold } \mathcal{M}, w, v \models_{\Sigma} \alpha \\
\mathcal{M}, w, v \models_{\Sigma} \alpha \vee \beta \quad \text { iff } & \mathcal{M}, w, v \models_{\Sigma} \alpha \text { or } \mathcal{M}, w, v \models_{\Sigma} \beta \\
\mathcal{M}, w, v \models_{\Sigma}(\exists x) \alpha & \text { iff } & \text { there exists } a \in S \text { such that } \mathcal{M}, w, v[x \mapsto a] \models_{\Sigma} \alpha \\
\mathcal{M}, w, v \models_{\Sigma} \diamond \alpha & \text { iff } & \text { there exists } w^{\prime} \in \Delta \text { such that }\left(w, w^{\prime}\right) \in \mathcal{R} \text { and } \\
\mathcal{M}, w^{\prime}, v \models_{\Sigma} \alpha
\end{array}
$$

the valuation function $m_{w, v}^{\mathcal{M}}: \operatorname{Term}(\Sigma, \mathcal{X}) \rightarrow S$, extending $v$ to terms, is defined as:

$$
\begin{aligned}
m_{w, v}^{\mathcal{M}}(c) & =c^{\mathcal{L}(w)}, \text { for all } c \in \mathcal{C} . \\
m_{w, v}^{\mathcal{M}}(x) & =v(x), \text { for all } x \in \mathcal{X} . \\
m_{w, v}^{\mathcal{M}}\left(f\left(t_{1}, \ldots, t_{\text {arity }(f)}\right)\right) & =f^{\mathcal{L}(w)}\left(m_{w, v}^{\mathcal{M}}\left(t_{1}\right), \ldots, m_{w, v}^{\mathcal{M}}\left(t_{\text {arity }(R)}\right)\right), \text { for all } f \in \mathcal{F} \text { and } \\
& \left\{t_{1}, \ldots, t_{\text {arity }(f)}\right\} \subseteq \operatorname{Term}(\Sigma, \mathcal{X}) .
\end{aligned}
$$

It is easy to prove that $\mathbb{I}_{F O M L}$ is an institution. Next, we present the tableau technique introduced by Fitting in [47, Chapter 5]. Given $\Sigma \in|\operatorname{Sign}|, \alpha, \beta \in \operatorname{Sen}(\Sigma)$ we define the following dual operators $(\forall x) \alpha \equiv \neg(\exists x) \neg \alpha$ and $\alpha \wedge \beta \equiv \neg(\neg \alpha \vee \neg \beta)$. A prefix is a finite sequence of positive integers. A prefixed formula is an expression of the form $\sigma: S$, where $\sigma$ is a prefix and $S$ is a set of formulae.

Let $\Sigma \in|\operatorname{Sign}|$ and $\Gamma \subseteq \operatorname{Sen}(\Sigma)$; then a tableau for $\Gamma$ is a tree defined as in Example 3.2 but considering that nodes in the tree are sets of prefixed formulae instead of just formulae. There are four new rules, two for possibility and two for necessity: 


$$
\begin{array}{cc}
{[n \text { is new }] \frac{X \cup\{\sigma: \diamond A\}}{X \cup\{\sigma: \diamond A, \sigma . n: A\}}\left[\text { Pos }_{1}\right] \quad[n \text { is new }] \frac{X \cup\{\sigma: \neg \square A\}}{X \cup\{\sigma: \neg \square A, \sigma . n: \neg A\}}\left[\text { Pos }_{2}\right]} \\
X \cup\{\sigma: \square A\} & X \cup\{\sigma: \neg \diamond A\} \\
\hline X \cup\{\sigma: \square A\} \cup\{\sigma . n: A \mid \sigma . n: B \in X\} & {\left[N e c_{1}\right] \quad}
\end{array}
$$

The notions of branch, saturated branch and closed branch are defined as in Example 3.2, but taking into account that formulae are prefixed, so closeness requires contradictory formulae to be named with the same prefix. Once again, given the way in which branches are defined, for a branch in a tableau, either its limit set is the entire set of sentences over the signature as a consequence of containing a contradiction named with the same prefix, or it exhibits, by means of a set of prefixed formulae, a class of models satisfying them. Defining a satisfiability calculus based on the technique presented before is analogous to Example 3.2, but considering the morphisms needed to represent the additional derivations using rules $\mathrm{Pos}_{1}, \mathrm{Pos}_{2}, \mathrm{Nec}_{1}$ and $\mathrm{Nec}_{2}$.

In order to define the satisfiability subcalculus corresponding to the term-free fragment of $\mathbb{I}_{F O M L}$, we have to provide appropriate definitions for $\operatorname{Sign}_{0}$ and ax. We define $\operatorname{Sign}_{0}$ as the complete subcategory of Sign whose sets of constant and function symbols are empty and whose set of predicate symbols contain only predicates with arity 0 . The reader should note that by choosing those signatures, the restriction of the functor Sen to them results in the term-free fragment of the language. Therefore, given $\Sigma=\langle\emptyset, \emptyset, \mathcal{P}\rangle \in\left|\operatorname{Sign}_{0}\right|, \operatorname{Sen}_{0}(\Sigma)$ is the subset of the smallest set $\operatorname{Form}(\Sigma, \emptyset)$ of the formulae obtained by the following grammar:

$$
\operatorname{Form}(\Sigma, \emptyset)::=P, P \in \mathcal{P}|\neg \operatorname{Form}(\Sigma, \emptyset)| \operatorname{Form}(\Sigma, \emptyset) \vee \operatorname{Form}(\Sigma, \emptyset) \mid \diamond \operatorname{Form}(\Sigma, \emptyset)
$$

containing only all the sentences (i.e., formulae with no free variables).

Finally, we define ax $=\mathscr{P} \circ \mathbf{S e n}_{0}$. Therefore, for any $\Sigma \in\left|\operatorname{Sign}_{0}\right|$, the application of the tableaux technique presented above, defined as a satisfiability subcalculus, is capable of building tableaux for any set of formulae over sentences of $\operatorname{Sen}_{0}(\Sigma)$. This is a direct consequence of [47, Defs. 2.2.1-2.2.8].

The next definition introduces the notion of effectiveness of the procedure for constructing structures characterising models for sets of sentences over a logical (sub)language. To this end, we follow Meseguer's approach [2, Def. 16], which, in turn, adopts the axiomatic view of computability outlined by Shoenfield in [49]. The elementary notions are those of a finite object, a space of finite objects, and recursive functions. In Shoenfield's own words, a finite object is an "object which can be specified by a finite amount of information", a space is "an infinite class $X$ of finite objects such that, given a finite object $x$, we can decide whether or not $x$ belongs to $X$ ". Now, given spaces $X$ and $Y$, a recursive function $f: X \rightarrow Y$ is then a total function that can be computed by an algorithm (i.e., by a terminating program, disregarding space and time limitations); spaces and recursive functions form a category Space. Effectiveness is then obtained by restricting sentences and axioms over selected signatures to be organised in a space.

\section{Definition 3.6. (Effective satisfiability subcalculus)}

A structure of the form $\mathbb{Q}=\left\langle\operatorname{Sign}, \operatorname{Sen}, \mathbf{M o d}, \operatorname{Sign}_{0}, \operatorname{Sen}_{0}, \mathbf{a x},\left\{\models^{\Sigma}\right\}_{\Sigma \in|\operatorname{Sign}|}, \mathbf{M}, \mathbf{M o d s}, \mu\right\rangle$ is a effective satisfiability subcalculus if it satisfies the following conditions:

- $\left\langle\right.$ Sign, Sen, Mod, $\left.\left\{\mid=^{\Sigma}\right\}_{\Sigma \in \mid \text { Sign } \mid}\right\rangle$ is an institution. 
- $\operatorname{Sign}_{0}$ is a subcategory of Sign called the subcategory of admissible signatures; let $J: \operatorname{Sign}_{0} \hookrightarrow$ Sign be the inclusion functor.

- $\operatorname{Sen}_{0}: \operatorname{Sign}_{0} \rightarrow$ Space is a functor such that $\mathcal{U} \circ \mathbf{S e n}_{0}=\operatorname{Sen} \circ J$, where $\mathcal{U}:$ Space $\rightarrow$ Set is the obvious forgetful functor.

- ax : $\operatorname{Sign}_{0} \rightarrow$ Space is a subfunctor of the functor obtained by composing $\mathbf{S e n}_{0}$ with the functor $\mathscr{P}_{\text {fin }}:$ Space $\rightarrow$ Space, that sends each space to the space of its finite subsets. This defines a subcategory $\mathrm{Th}_{a x}$ of Th whose objects are theories $T=\langle\Sigma, \Gamma\rangle$ with $\Sigma \in \operatorname{Sign}_{0}$ and $\Gamma \in \operatorname{ax}(\Sigma)$, and whose morphisms are axiom-preserving theory morphisms $H$ such that $H$ is in $\operatorname{Sign}_{0}$.

- $\mathbf{M}: \mathrm{Th}_{a x}{ }^{\mathrm{op}} \rightarrow$ Struct $_{S C}$ is a functor. Let $T \in\left|\mathrm{Th}_{a x}\right|$, then $\mathbf{M}(T) \in \mid$ Struct $_{S C} \mid$ is the model structure of $T$.

- Mods : Struct $S_{C} \rightarrow$ Space is a functor. Let $T \in\left|\mathrm{Th}_{a x}\right|$, then $\operatorname{Mods}(\mathbf{M}(T))$ is the set of canonical models of $T$; the composite functor Mods $\circ \mathbf{M}: \mathrm{Th}_{a x}{ }^{\mathrm{op}} \rightarrow$ Space will be denoted by models.

- $\mu$ : models $\dot{\rightarrow} \mathscr{P} \circ$ Mod is a natural transformation such that, for each $T=\langle\Sigma, \Gamma\rangle \in\left|\mathrm{Th}_{0}{ }^{\mathrm{op}}\right|$, the image of $\mu_{T}: \operatorname{models}(T) \rightarrow \mathscr{P} \circ \operatorname{Mod}(T)$ is the subcategory of $\operatorname{Mod}(T)$ corresponding to each canonical representation of a class of models in $|\operatorname{model} s(T)|$. The map $\mu_{T}$ is called the projection of the category of models of the theory $T$.

- $\mathcal{U}(\mathbb{Q})=\left\langle\operatorname{Sign}\right.$, Sen, Mod, $\operatorname{Sign}_{0}, \mathcal{U} \circ$ ax, $\left\{\models^{\Sigma}\right\}_{\Sigma \in|\operatorname{Sign}|}, \mathbf{M}, \mathcal{U} \circ$ Mods, $\left.\mu \circ \mathcal{U}\right\rangle$ is a satisfiability subcalculus, where $\mu \circ \mathcal{U}$ denotes the natural transformation formed by $\left\{\mu_{T} \circ \mathcal{U}\right.$ : $\mathcal{U} \circ \operatorname{models}(T) \rightarrow \mathscr{P} \circ \operatorname{Mod}(T)\}_{T \in\left|\mathrm{T}_{a x}\right|}$.

Concisely speaking, a space is an infinite set of finite objects for which membership can be decided. Resorting to this, the main differences between Definitions 3.4 and 3.6 are that:

1. the restriction $\operatorname{Sen}_{0}$ of the functor Sen must satisfy $\mathcal{U} \circ \mathbf{S e n}_{0}=\operatorname{Sen} \circ J$, and

2. all occurrences of the category Set involved in the definition are replaced by Space (see for example, the conditions imposed on functor ax).

Condition 1 above establishes that the restriction $\mathbf{S e n}_{0}: \operatorname{Sign}_{0} \rightarrow$ Space of the functor Sen : Sign $\rightarrow$ Set is coherent with the inclusion functor $J$ and the forgetful functor $\mathcal{U}$, and Condition 2 ensures decidability.

The following example is formulated as an extension of Example 3.5 by proving the effectiveness of the satisfiability subcalculus for finite sets of axioms over the term-free fragment of first-order modal logic.

Example 3.7. (Effectiveness of the Sat. Subcalculus for Finite Presentations over the Term-Free Restriction of First-Order Modal Logic) 
Consider $\mathbb{Q}=\left\langle\operatorname{Sign}\right.$, Sen, Mod, Sign 0 , Sen 0 , ax, $\left\{\mid=^{\Sigma}\right\}_{\Sigma \in|\operatorname{Sign}|}, \mathbf{M}$, Mods, $\left.\mu\right\rangle$ the satisfiability subcalculus determined by the restriction of first-order modal logic to its term-free fragment, presented in Example 3.5. Its effectiveness can be derived by setting the functor ax to be $\mathscr{P}_{f i n} \circ \mathbf{S e n}_{0}$ in order to guarantee that any tableau will also be finite, and with the following observations:

- any tableau obtained by applying the technique presented in [47] for first-order modal logic to any theory presentation over $\operatorname{ax}(\Sigma)$, for any $\Sigma \in\left|\operatorname{Sign}_{0}\right|$, is equivalent to one obtained by applying the tableaux technique for modal logic [50];

- the definition of satisfiable set of prefixed formulae [47, Def. 2.5.1] and the fact that a closed tableau is not satisfiable [47, Prop. 2.5.2];

- soundness of the rules: the definition of extension of a satisfiable branch and the fact that it results in a satisfiable branch [47, Prop. 2.5.3]; and

- completeness of the procedure: the fact that every finite set of prefixed formulae has a bounded height, finitely branching saturated tableau [50, Chap. 8].

As a way of illustration of the previous definition, we provide a proof of the effectiveness of $\mathbb{Q}$ by instantiating the formal definition of effective satisfiability subcalculus given in Definition 3.6.

$\operatorname{Sign}_{0}$ is a complete subcategory of Sign so we assume $J: \operatorname{Sign}_{0} \hookrightarrow \operatorname{Sign}$ to be the identity inclusion functor (i.e., for all $\Sigma \in\left|\operatorname{Sign}_{0}\right|, J(\Sigma)=\Sigma$ and for all $\sigma \in\left\|\operatorname{Sign}_{0}\right\|, J(\sigma)=\sigma$. $\operatorname{Sen}_{0}$ is defined as the subfunctor of Sen resulting from restricting the latter to the objects and morphisms in $\operatorname{Sign}_{0}$ ). It is easy to prove that the image of $\mathbf{S e n}_{0}:$ Sign $\rightarrow$ Space is a space because:

- given $\Sigma \in\left|\operatorname{Sign}_{0}\right|$, the set $\operatorname{Sen}_{0}(\Sigma)$ is a recursive infinite set of finite objects, and

- given $\sigma \in\left\|\operatorname{Sign}_{0}\right\|$, $\operatorname{Sen}_{0}(\sigma)$ is a total function mapping formulae to formulae of the corresponding spaces in such a way that identities and composition are preserved (see Lemma A.15 for a detailed proof).

It is also easy to observe that $\mathcal{U} \circ \mathbf{S e n}_{0}=\operatorname{Sen} \circ J$ as a consequence of:

- $J: \operatorname{Sign}_{0} \hookrightarrow$ Sign being the identity inclusion functor,

- the nature of the forgetful functor $\mathcal{U}:$ Space $\rightarrow$ Set, and

- $\mathbf{S e n}_{0}$ being the subfunctor of Sen, when the latter is restricted to the objects and morphisms of $\operatorname{Sign}_{0}$ (see Lemma A.16 for a detailed proof).

Next, we prove that ax : $\operatorname{Sign}_{0} \rightarrow$ Space is a functor as a consequence of being the extension of $\mathbf{S e n}_{0}$ to finite sets of formulae (see Lemma A.17 for a detailed proof). Finally, we note that Mods : Struct $S C \rightarrow$ Space is a functor; since Mods is the extension of ax to finite trees whose nodes are sets of formulae (see Lemma A.18 for the details). 


\section{Relating Satisfiability Calculi}

Several notions of morphisms between institutions, and their properties, were investigated in [4, 2, 5]. More recently, in [6], all these notions of morphism were investigated in more detail by observing how the direction of the arrows modify its interpretation. In this section we will concentrate only on extending the results presented by Tarlecki in [5], focussing on institution morphisms and comorphisms, since these notions have been used to formalise several concepts arising in software engineering: they are used as the main vehicle for borrowing proofs along logic translation in [5]; for defining heterogeneous development environments for software specifications and designs in [10,51], which provides the foundations of tools like HETS [14] and CafeOBJ [11]; for providing structured specifications in general in [7], and for specific formal languages in [52, 53]; for defining proof systems for structured specifications $[54,55,56]$; and for formalising data and specification refinements in $[33,57,52]$, just to give a few examples.

Let us recall the basic definitions regarding institution morphism and comorphism.

\section{Definition 4.1. (Institution comorphism [5])}

Let $\mathbb{I}=\left\langle\right.$ Sign, Sen, Mod, $\left.\left\{\left.\right|_{\Sigma}\right\}_{\Sigma \in|\operatorname{Sign}|}\right\rangle$ and $\mathbb{I}^{\prime}=\left\langle\operatorname{Sign}^{\prime}, \mathbf{S e n}^{\prime}, \mathbf{M o d}^{\prime},\left\{\left.\right|_{\Sigma} ^{\prime}\right\}_{\Sigma \in\left|\operatorname{Sign}^{\prime}\right|}\right\rangle$ be institutions. Then, $\left\langle\rho^{\text {Sign }}, \rho^{\text {Sen }}, \rho^{\text {Mod }}\right\rangle: \mathbb{I} \rightarrow \mathbb{I}^{\prime}$ is an institution comorphism if and only if:

- $\rho^{\text {Sign }}:$ Sign $\rightarrow \operatorname{Sign}^{\prime}$ is a functor,

- $\rho^{S e n}:$ Sen $\rightarrow$ Sen $^{\prime} \circ \rho^{\text {Sign }}$ is a natural transformation, being a family of total functions $\left\{\rho_{\Sigma}^{\text {Sen }}: \operatorname{Sen}(\Sigma) \rightarrow \operatorname{Sen}^{\prime}\left(\rho^{\operatorname{Sign}}(\Sigma)\right)\right\}_{\Sigma \in|\operatorname{Sign}|}$ such that for signatures $\Sigma_{1}, \Sigma_{2} \in|\operatorname{Sign}|$ and morphism $\sigma: \Sigma_{1} \rightarrow \Sigma_{2} \in\|\operatorname{Sign}\|$,

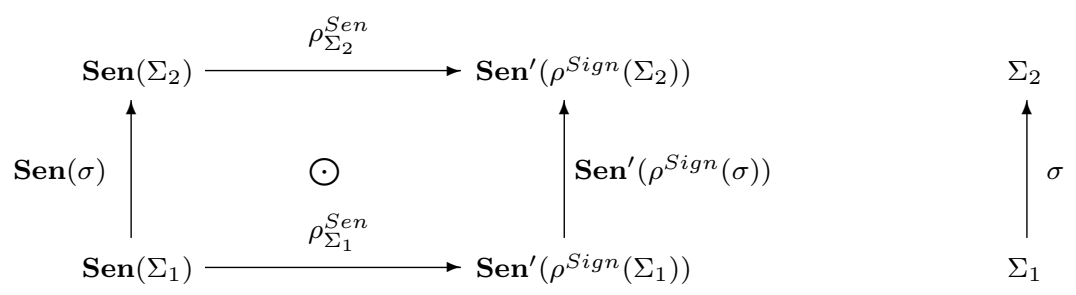

- $\rho^{M o d}: \mathbf{M o d}^{\prime} \circ\left(\rho^{\text {Sign }}\right)^{\text {op }} \rightarrow \mathbf{M o d}^{5}$ is a natural transformation; being a family of functors $\left\{\rho_{\Sigma}^{\text {Mod }}: \operatorname{Mod}^{\prime}\left(\left(\rho^{\text {Sign }}\right)^{\text {op }}(\Sigma)\right) \rightarrow \operatorname{Mod}(\Sigma)\right\}_{\Sigma \in|\operatorname{Sign}|}$, such that for signatures $\Sigma_{1}, \Sigma_{2} \in \mid$ Sign $\mid$ and morphism $\sigma: \Sigma_{1} \rightarrow \Sigma_{2} \in \|$ Sign $\|$,

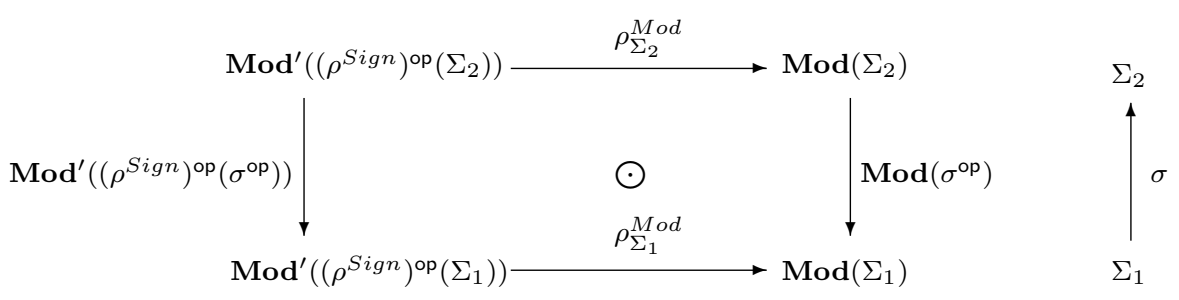

\footnotetext{
${ }^{5}$ The functor $\left(\rho^{\text {Sign }}\right)^{\text {op }}: \operatorname{Sign}^{\prime \text { op }} \rightarrow \operatorname{Sign}^{\text {op }}$ is the same as $\rho^{\text {Sign }}: \operatorname{Sign}^{\prime} \rightarrow$ Sign but considered between the opposite categories.
} 
such that for any $\Sigma \in|\operatorname{Sign}|$, the function $\rho_{\Sigma}^{S e n}: \operatorname{Sen}(\Sigma) \rightarrow \operatorname{Sen}^{\prime}\left(\rho^{\operatorname{Sign}}(\Sigma)\right)$ and the functor $\rho_{\Sigma}^{\text {Mod }}:$ $\operatorname{Mod}^{\prime}\left(\rho^{\operatorname{Sign}}(\Sigma)\right) \rightarrow \operatorname{Mod}(\Sigma)$ preserves the following satisfaction condition: for any $\alpha \in \operatorname{Sen}(\Sigma)$ and $\mathcal{M}^{\prime} \in\left|\operatorname{Mod}\left(\rho^{\operatorname{Sign}}(\Sigma)\right)\right|$,

$$
\mathcal{M}^{\prime} \mid=^{\rho^{S i g n}}(\Sigma) \rho_{\Sigma}^{\text {Sen }}(\alpha) \text { iff } \rho_{\Sigma}^{M o d}\left(\mathcal{M}^{\prime}\right) \mid=^{\Sigma} \alpha .
$$

Intuitively, an institution comorphism $\rho: \mathbb{I} \rightarrow \mathbb{I}^{\prime}$ expresses how the "poorer" set of sentences (respectively, category of models) associated with $\mathbb{I}$ is encoded into the "richer" one associated with $\mathbb{I}^{\prime}$. This is done by providing: 1. a mapping from $\mathbb{I}$ signatures to $\mathbb{I}^{\prime}$ signatures, 2. a translation of $\mathbb{I}$-sentences on signature $\Sigma$ to $\mathbb{I}^{\prime}$-sentences on the signature to which $\Sigma$ is mapped, 3. a reduction of $\mathbb{I}^{\prime}$-models for the signature to which $\Sigma$ is mapped to $\mathbb{I}$-models for signature $\Sigma$. The direction of the arrows shows how the whole of $\mathbb{I}$ is represented by some parts of $\mathbb{I}^{\prime}$. Institution comorphisms enjoy some interesting properties, for instance, the preservation of logical consequence [5, Prop. 13], and, under some conditions, its reflection [5, Thm. 14]. The interested reader is referred to [5] for further details about these properties, and to $[58,59]$ for some structural properties of the category formed by institutions and institution comorphisms.

In many cases, in particular those in which the class of models of a signature in the source institution is completely axiomatisable in the language of the target one, Definition 4.1 can easily be extended to map signatures of one institution to theories of another. This is done in such a way that the class of models of the richer logical language can be constrained, by means of the addition of axioms (therefore, there is a need for theories in the image of the functor $\gamma^{\text {Sign }}$ ), to represent exactly the same class of models of the signature in the poorer logical language. Many examples of this relation between logics have been developed, one of the most prominent examples being the translation from modal logic to first-order logic with equality [60]. Many other examples are worth mentioning, such as algebraisations of different logics, like those relating fork algebras [61, 62], an extension of relation algebras [63, 64], with several other logics (c.f. [37, 65]).

In some cases institution comorphisms can be extended to what Meseguer introduced under the name map of institutions [2, Def. 27], and recently renamed as theoroidal comorphism [6, Def. 5.3], by reformulating the definition so that the functor mapping signatures to theory presentations maps theory presentation to theory presentations (i.e. $\gamma^{T h}: \mathrm{Th} \rightarrow \mathrm{Th}^{\prime}$ ). This extension can be done in many ways, one of them being when $\gamma^{T h}$ is $\gamma^{S e n}$-sensible (see Definition 2.4) with respect to the entailment systems induced by the institutions $\mathbb{I}$ and $\mathbb{I}^{\prime}$.

The notion of a theoroidal comorphism of satisfiability calculi is the natural extension of a theoroidal comorphism of institutions in order to consider the more concrete version of the satisfiability relation. In some sense, if a theoroidal comorphism of institutions provides a means for representing one satisfiability relation in terms of another, in a semantics preserving way, the theoroidal comorphism of satisfiability calculi provides a means for understanding one particular model construction technique, for a (potentially) poorer logical system, in terms of the structures produced by another, designed for a (potentially) richer logical language.

\section{Definition 4.2. (Theoroidal comorphism of satisfiability calculi)}

Let $\mathbb{S}$ be the satisfiability calculus $\left\langle\right.$ Sign, Sen, Mod, $\left.\left\{\models^{\Sigma}\right\}_{\Sigma \in|\operatorname{Sign}|}, \mathbf{M}, \operatorname{Mods}, \mu\right\rangle$ and $\mathbb{S}^{\prime}$ the satisfiability calculus $\left\langle\operatorname{Sign}^{\prime}, \mathbf{S e n}^{\prime}, \mathbf{M o d}^{\prime},\left\{\models^{\prime \Sigma}\right\}_{\Sigma \in\left|\operatorname{Sign}^{\prime}\right|}, \mathbf{M}^{\prime}, \mathbf{M o d s}^{\prime}, \mu^{\prime}\right\rangle$. 
Then, $\left\langle\rho^{S i g n}, \rho^{S e n}, \rho^{M o d}, \gamma\right\rangle: \mathbb{S} \rightarrow \mathbb{S}^{\prime}$ is a theoroidal comorphism of satisfiability calculi if and only if:

- $\left\langle\rho^{T h}, \rho^{S e n}, \rho^{M o d}\right\rangle: \mathbb{I} \rightarrow \mathbb{I}^{\prime}$ is an institution theoroidal comorphism, where $\rho^{T h}: \mathrm{Th} \rightarrow \mathrm{Th}^{\prime}$ is the $\rho^{S e n}$-sensible extension of $\rho^{\text {Sign }}$ defined as $\rho^{T h}(\langle\Sigma, \Gamma\rangle)=\left\langle\rho^{\operatorname{Sign}}(\Sigma), \emptyset^{\prime} \cup \rho_{\Sigma}^{S e n}(\Gamma)\right\rangle$, and

- $\gamma:$ models $^{\prime} \circ \rho^{T h} \dot{\rightarrow}$ models is a natural transformation such that the following equality holds:
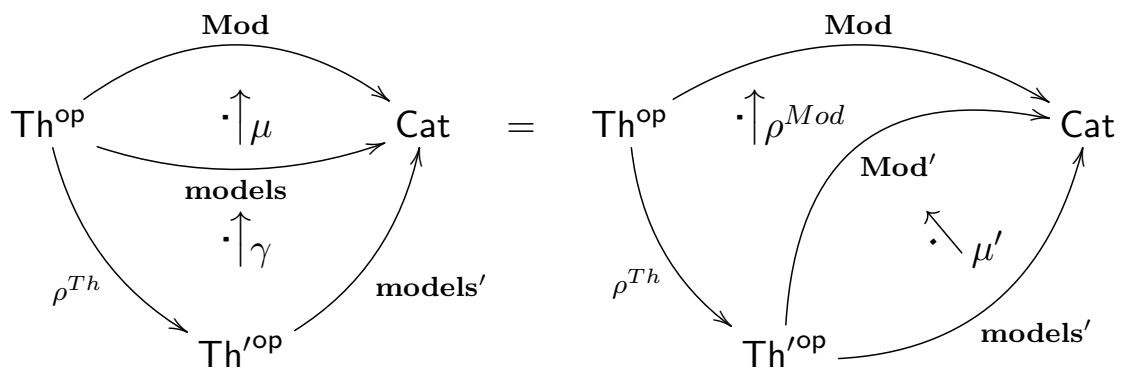

Roughly speaking, the 2-cell equality in the definition says that, if we obtain counterexamples by resorting to the machinery of $\mathbb{S}^{\prime}$ and then applying $\rho$ to extract models of $\mathbb{S}$, the resulting structures are those that can be obtained by employing the machinery of $\mathbb{S}$. The following example illustrates these ideas.

\section{Example 4.3. (Relating Modal Logic to First-Order Logic)}

A simple example of a theoroidal comorphism between satisfiability calculi is the relation between the tableau method for propositional logic and the one for first-order logic. Let us introduce a more interesting example. We will map the tableau method for modal logic (as presented by Fitting [38]) to the first-order predicate logic tableau method. The theoroidal comorphism relating the institutions is given by the standard translation from modal logic to first-order logic [60, Def. 2.45] (recalled below). Let us recast here the tableau method for the system K of modal logic. Recall that formulae of standard modal logic are built from boolean operators and the "diamond operator" $\diamond$. The "box operator" $\square$ is defined as the dual to $\diamond$, i.e., as $\square \varphi=\neg \diamond \neg \varphi$. Intuitively, formula $\diamond \varphi$ states that $\varphi$ holds in some state accessible from the current state, while $\square \varphi$ states that $\varphi$ holds in all states accessible from the current state. The semantics for modal logic is given by means of Kripke structures. A Kripke structure is a tuple $\langle W, R, L\rangle$, where $W$ is a set of states, $R \subseteq W \times W$ is a relation between states, and $L: W \rightarrow 2^{A P}$ is a labelling function ( $A P$ is a set of atomic propositions). Note that a signature in modal logic is given by a set of propositional letters: $\left\langle\left\{p_{i}\right\}_{i \in \mathcal{I}}\right\rangle$; the interested reader is referred to [60]. In [38] modal formulae are prefixed by labels denoting semantic states. Labelled formulae are then terms of the form $\ell: \varphi$, where $\varphi$ is a modal formula and $\ell$ is a sequence of natural numbers $n_{0}, \ldots, n_{k}$. The relation $R$ between these labels is then defined in the following way: $\ell R \ell^{\prime} \equiv \exists n: \ell, n=\ell^{\prime}$. The new rules are the following:

$$
\text { For all } \ell^{\prime} \text { s.t. } \ell R \ell^{\prime} \text { and s.t. } \ell^{\prime} \text { appears in } X \frac{X \cup\{\ell: \square \varphi\}}{X \cup\left\{\ell: \square \varphi, \ell^{\prime}: \varphi\right\}}[\square] \quad \text { For } \ell^{\prime} \text { such that } \ell R \ell^{\prime} \frac{X \cup\{\ell: \diamond \varphi\}}{X \cup\left\{\ell: \diamond \varphi, \ell^{\prime}: \varphi\right\}}[\diamond]
$$

The rules for the propositional connectives are the usual ones, obtained by labelling the formulae. Note that labels denote states of a Kripke structure. Branches, saturated branches and closed branches are defined in the same way as in Example 3.2, but considering the relations between sets to be also 
indexed by the relation used at that point. Thus, $s_{i} \underset{R_{i}}{\stackrel{\tau_{\alpha_{i}}}{\longrightarrow}} s_{i+1}$ must be understood as follows: the set $s_{i+1}$ is obtained from $s_{i}$ by applying rule $\tau_{\alpha_{i}}$ to formula $\alpha_{i} \in s_{i}$ under the accessibility relation $R_{i}$.

Let $\mathbb{S C}_{F O L}=\left\langle\operatorname{Sign}_{F O L}, \operatorname{Sen}_{F O L}, \operatorname{Mod}_{F O L},\left\{1={ }_{F O L}^{\Sigma}\right\}_{\Sigma \in \mid \operatorname{Sign}_{F O L}}, \mathbf{M}_{F O L}, \mathbf{M o d s}_{F O L}, \mu_{F O L}\right\rangle$ and $\mathbb{S C}_{K}=\left\langle\operatorname{Sign}_{K}, \operatorname{Sen}_{K}, \operatorname{Mod}_{K},\left\{\mid={ }_{K}^{\Sigma}\right\}_{\Sigma \in\left|\operatorname{Sign}_{K}\right|}, \mathbf{M}_{K}, \operatorname{Mods}_{K}, \mu_{K}\right\rangle$, be the classic tableau methods for first-order predicate logic and modal logic, respectively, formalised as satisfiability calculi. First we define a comorphism $\left\langle\rho^{S i g n}, \rho^{S e n}, \rho^{M o d}\right\rangle: \mathbb{I}_{K} \rightarrow \mathbb{I}_{F O L}$ as follows:

- $\rho^{\text {Sign }}: \operatorname{Sign}_{K} \rightarrow \operatorname{Sign}_{F O L}$ is defined as $\rho^{\operatorname{Sign}}\left(\left\langle\left\{p_{i}\right\}_{i \in \mathcal{I}}\right\rangle\right)=\left\langle R,\left\{p_{i}\right\}_{i \in \mathcal{I}}\right\rangle$ by mapping each propositional variable $p_{i}$ to a first-order unary logic predicate $p_{i}$, for all $i \in \mathcal{I}$, and adding a binary predicate $R$, and $\rho^{\operatorname{Sign}}\left(\sigma:\left\langle\left\{p_{i}\right\}_{i \in \mathcal{I}}\right\rangle \rightarrow\left\langle\left\{p_{i^{\prime}}^{\prime}\right\}_{i^{\prime} \in \mathcal{I}^{\prime}}\right\rangle\right)=\sigma^{\prime}:\left\langle R,\left\{p_{i}\right\}_{i \in \mathcal{I}}\right\rangle \rightarrow$ $\left\langle R^{\prime},\left\{p_{i^{\prime}}^{\prime}\right\}_{i^{\prime} \in \mathcal{I}^{\prime}}\right\rangle$ mapping $R$ to $R^{\prime}$, and $p_{i}$ to $\sigma\left(p_{i}\right)$ for all $i \in \mathcal{I}$, and we prove it is a functor (see Lemma A.20 for a detailed proof),

- Let $\Sigma \in\left|\operatorname{Sign}_{K}\right|$. Then $\rho_{\Sigma}^{\text {Sen }}: \operatorname{Sen}_{K}(\Sigma) \rightarrow \operatorname{Sen}_{F O L}\left(\rho^{\operatorname{Sign}}(\Sigma)\right)$ is defined recursively as $\rho_{\Sigma}^{S e n}(\alpha)=T_{\Sigma, x}(\alpha)$ where:

$$
\begin{aligned}
T_{\Sigma, x}(p) & =p(x), \text { for all } p \in \Sigma . \\
T_{\Sigma, x}(\neg \alpha) & =\neg T_{\Sigma, x}(\alpha) \\
T_{\Sigma, x}(\alpha \vee \beta) & =T_{\Sigma, x}(\alpha) \vee T_{\Sigma, x}(\beta) \\
T_{\Sigma, x}(\diamond \alpha) & =(\exists y)\left(R(x, y) \wedge T_{\Sigma, y}(\alpha)\right)
\end{aligned}
$$

and considering that for every $\Sigma \in\left|\operatorname{Sign}_{K}\right|, \rho_{\Sigma}^{S e n}$ is a function, we prove that $\rho^{S e n}$ is a natural family of functions (see Lemma A.23 for a detailed proof), and

- Let $\Sigma=\left\langle\left\{p_{i}\right\}_{i \in \mathcal{I}}\right\rangle \in\left|\operatorname{Sign}_{K}\right|, \rho_{\Sigma}^{M o d}: \operatorname{Mod}_{F O L}\left(\rho^{\operatorname{Sign}}(\Sigma)\right) \rightarrow \operatorname{Mod}_{K}(\Sigma)$ be defined as follows:

- for all $\mathcal{M}=\left\langle S, \bar{R},\left\{\bar{p}_{i}\right\}_{i \in \mathcal{I}}\right\rangle \in\left|\operatorname{Mod}_{F O L}\left(\left\langle R,\left\{p_{i}\right\}_{i \in \mathcal{I}}\right\rangle\right)\right|, \rho_{\Sigma}^{M o d}(\mathcal{M})=\langle S, \bar{R}, L\rangle$, with $L\left(p_{i}\right)=\left\{s \in S \mid \overline{p_{i}}(s)\right\}$. $^{6}$

- let $\Sigma=\left\langle\left\{p_{i}\right\}_{i \in \mathcal{I}}\right\rangle \in\left|\operatorname{Sign}_{K}\right|$; then for all $h:\left\langle S_{1}, \overline{R_{1}},\left\{\overline{p_{1 i}}\right\}_{i \in \mathcal{I}}\right\rangle \rightarrow\left\langle S_{2}, \overline{R_{2}},\left\{\overline{p_{2 i}}\right\}_{i \in \mathcal{I}}\right\rangle \in$ $\left\|\operatorname{Mod}_{F O L}\left(\left\langle R,\left\{p_{i}\right\}_{i \in \mathcal{I}}\right\rangle\right)\right\|$, we define $\rho_{\Sigma}^{M o d}(h)$ to be $\widehat{h}$, where $\widehat{h}\left(s_{1}\right)=s_{2}$ if and only if $h\left(s_{1}\right)=s_{2}$ for all $s_{1} \in S_{1}$.

and then prove that: 1 . for every $\Sigma \in\left|\operatorname{Sign}_{K}\right|, \rho_{\Sigma}^{M o d}$ is a functor (see Lemma A.26), and 2. $\rho^{M o d}$ is a natural family of functors (see Lemma A.27).

As a corollary of the previous observations, we obtain that $\left\langle\rho^{T h}, \rho^{S e n}, \rho^{M o d}\right\rangle$, where $\rho^{T h}$ is the $\rho^{S e n}$ sensible extension of $\rho^{S i g n}$ given in Definition 4.2, is a theoroidal comorphism of institutions. The proof of this corollary ultimately relies on the correctness of the translation presented in [60]. Using this map we can define a theoroidal comorphism between the corresponding satisfiability calculi. Now, we have to prove that structures, representing the tableaux for first-order predicate logic, for formulae

\footnotetext{
${ }^{6}$ Recall that given $\Sigma=\left\langle\left\{p_{i}\right\}_{i \in \mathcal{I}}\right\rangle \in\left|\operatorname{Sign}_{K}\right|, \rho^{\operatorname{Sign}}(\Sigma)=\left\langle R,\left\{p_{i}\right\}_{i \in \mathcal{I}}\right\rangle$.
} 
resulting from the $\rho^{S e n}$-translation of modal logic formulae, can indeed be translated to modal logic tableaux for the original modal logic formulae.

Second, it is important to recall that, whenever we consider sets of formulae resulting from the application of function $\rho_{\left\langle\left\{p_{i}\right\}_{i \in \mathcal{I}}\right\rangle}^{S e n}$, the first-order predicate logic tableau will have a particular shape, mainly because the application of rule $[\forall]$ (resp., $[\exists]$ ) is restricted to the formulae resulting from the application of the translation. Thus, if $\left\langle\left\{p_{i}\right\}_{i \in \mathcal{I}}\right\rangle \in\left|\operatorname{Sign}_{K}\right|$, then for all $\alpha \in\left|\operatorname{Sen}_{K}\left(\left\langle\left\{p_{i}\right\}_{i \in \mathcal{I}}\right\rangle\right)\right|$, when $\rho_{\left\langle\left\{p_{i}\right\}_{i \in \mathcal{I}}\right\rangle}^{S e n}(\alpha)=\beta$, any quantified subformulae in $\beta$ is either of the form: $\left.a\right)(\forall x)(R(y, x) \Longrightarrow \varphi(x))$, or $b)(\exists x)(R(y, x) \wedge \varphi(x))$.

Then, we can obtain modal logic tableaux from first-order predicate logic tableaux by defining a function $T$ in the following way ${ }^{7,8}$ :

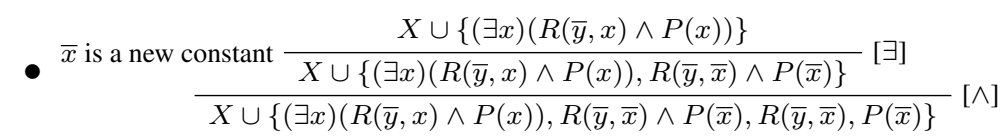

$$
\begin{aligned}
& \downarrow T \\
& \bar{x} \text { is a new label such that } R(\bar{y}, \bar{x}) \frac{X \cup\{\ell: \diamond P\}}{X \cup\{\ell: \diamond P, \bar{x}: P\}}[\diamond]
\end{aligned}
$$

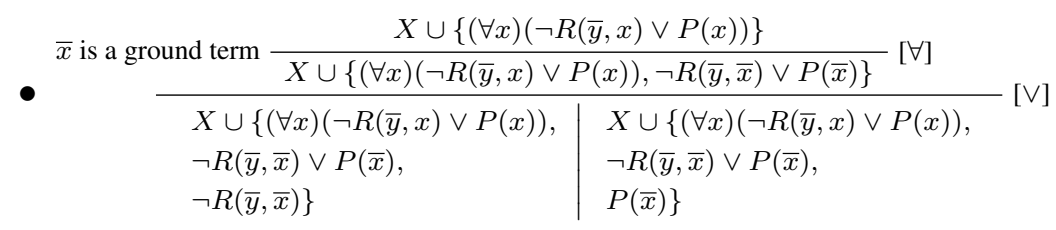

$$
\begin{aligned}
& \downarrow T \\
& \bar{x} \text { is a label occurring in } X \cup\{\ell\} \text { such that } R(\ell, \bar{x}) \frac{X \cup\{\ell: \square P\}}{X \cup\{\ell: \square P, \bar{x}: P\}}[\square]
\end{aligned}
$$

Third, we define the components of $\gamma: \operatorname{models}_{F O L} \circ \rho^{T h} \dot{\rightarrow} \operatorname{models}_{K}$. Let $\left\langle\left\langle\left\{p_{i}\right\}_{i \in \mathcal{I}}\right\rangle, \Gamma\right\rangle \in$ $\left|\mathrm{Th}^{K}\right|$, then we define:

$$
\gamma_{\left\langle\left\langle\left\{p_{i}\right\}_{i \in \mathcal{I}\rangle, \Gamma\rangle}\right.\right.}\left(\left\langle\left\langle R,\left\{p_{i}\right\}_{i \in \mathcal{I}}\right\rangle, \Delta\right\rangle\right)=\left\langle\left\langle\left\{p_{i}\right\}_{i \in \mathcal{I}}\right\rangle,\left\{\varphi \in\left|\operatorname{Sen}_{K}\left(\left\langle\left\{p_{i}\right\}_{i \in \mathcal{I}}\right\rangle\right)\right| \mid \rho_{\left\langle\left\{p_{i}\right\}_{i \in \mathcal{I}}\right\rangle}(\varphi) \in \Delta\right\}\right\rangle
$$

Observe that it is a functor and then prove that $\gamma: \operatorname{models}_{F O L} \circ \rho^{T h} \dot{\rightarrow} \operatorname{models}_{K}$ is a natural transformation (see Lemma A.33).

Fourth, we prove that $\mu_{K\left\langle\left\langle\left\{p_{i}\right\}_{i \in \mathcal{I}}\right\rangle, \Gamma\right\rangle} \circ \gamma_{\left\langle\left\langle\left\{p_{i}\right\}_{i \in \mathcal{I}}\right\rangle, \Gamma\right\rangle}=\rho_{\rho^{S i g n}\left(\left\langle\left\{p_{i}\right\}_{i \in \mathcal{I}}\right\rangle\right)}^{\operatorname{Mod}} \circ \mu_{F O L} \rho^{T h}\left(\left\langle\left\langle\left\{p_{i}\right\}_{i \in \mathcal{I}}\right\rangle, \Gamma\right\rangle\right)$ as required by Definition 4.2 (see Lemma A.34).

Finally, we obtain as a corollary that $\left\langle\rho^{T h}, \rho^{S e n}, \rho^{M o d}, \gamma\right\rangle$ is a theoroidal comorphism of satisfiability calculi.

\footnotetext{
${ }^{7}$ In order to simplify the following definition, we will restrict ourselves to those first-order predicate logic tableaux in which, when the rule $[\forall]$ (resp., $[\exists]$ ) is applied, the rules $[\vee]$ and $[\neg]$ (resp., $[\wedge]$ ) are applied. Notice that this assumption does not limit the definitions and results in any way because any other legal tableau for the same set of formulae that does not satisfy this property can be reordered to satisfy it.

${ }^{8}$ Notice that the translation of the rules for the propositional operators act on the labellings by just preserving them.
} 
This means that building a tableau using the first-order rules for the translation of a modal theory, then obtaining structures representing models in modal logic using $\gamma$, and therefore obtaining the class of models by using $\mu$, is exactly the same as obtaining the first-order models by $\mu^{\prime}$ and then the corresponding modal models by using $\rho^{M o d}$. Roughly speaking, this implies that the translation of saturated tableaux is coherent with respect to the mapping of institutions.

Theoroidal comorphisms of satisfiability calculi can be extended to theoroidal comorphisms of satisfiability subcalculi and theoroidal comorphisms of effective satistfiability subcalculi in a way analogous to [2, Def. 35], where the relation is established only between the restrictions performed over the calculus by the newly introduced elements.

\section{Definition 4.4. (Theoroidal comorphism of (effective) satisfiability subcalculi)}

Let $\mathbb{S}$ be the satisfiability calculus $\left\langle\right.$ Sign, Sen, Mod, $\left\{\models^{\Sigma}\right\}_{\Sigma \in|\operatorname{Sign}|}, \mathbf{M}$, Mods, $\left.\mu\right\rangle$ and $\mathbb{S}^{\prime}$ the satisfiability calculus $\left\langle\operatorname{Sign}^{\prime}, \mathbf{S e n}^{\prime}, \mathbf{M o d}^{\prime},\left\{\models^{\prime \Sigma}\right\}_{\Sigma \in\left|\operatorname{Sign}^{\prime}\right|}, \mathbf{M}^{\prime}, \mathbf{M o d s}^{\prime}, \mu^{\prime}\right\rangle$.

Then, $\left\langle\rho^{S i g n}, \rho^{S e n}, \rho^{M o d}, \gamma\right\rangle: \mathbb{S} \rightarrow \mathbb{S}^{\prime}$ is a theoroidal comorphism of satisfiability subcalculi if and only if:

- $\left\langle\rho^{T h}, \rho^{S e n}, \rho^{M o d}\right\rangle: \mathbb{I} \rightarrow \mathbb{I}^{\prime}$ is an institution theoroidal comorphism, where $\rho^{T h}: \mathrm{Th} \rightarrow \mathrm{Th}^{\prime}$ is a $\rho^{\text {Sen }}$-sensible extension of $\rho^{\text {Sign }}$,

- $\rho^{T h}: \mathrm{Th} \rightarrow \mathrm{Th}^{\prime}$ restricts to a functor $\rho_{a x}^{T h}: \mathrm{Th}_{a x} \rightarrow \mathrm{Th}_{a x}^{\prime}{ }^{9}$, and

- $\gamma:$ models $^{\prime} \circ \rho^{T h} \dot{\rightarrow}$ models is a natural transformation such that the following equality holds:
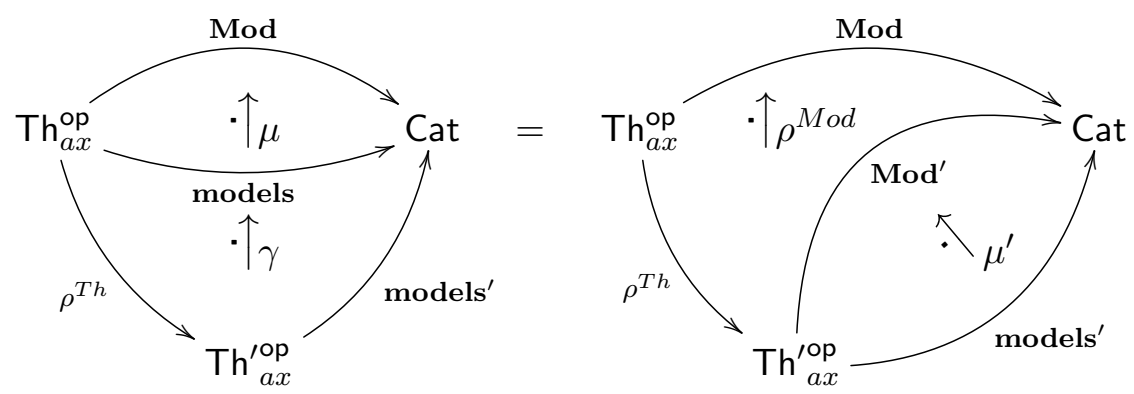

Theoroidal comorphisms of effective satisfiability subcalculi are defined exactly in the same way as theoroidal comorphisms of satisfiability subcalculi, but assuming that sets are spaces and not modifying the conditions required for the map. The restriction of the admissible formulae given by subfunctor $\mathbf{S e n}_{0} \subseteq$ Sen remains hidden in the use of categories of theories restricted to the particular spaces of formulae determined by functor ax.

The intuition behind the previous definition is that theoroidal comorphisms between (effective) satisfiability subcalculi only care about appropriately mapping the restriction of interest, introduced

\footnotetext{
${ }^{9}$ This means that functor $\rho^{T h}$ transforms theories (resp., axiom-preserving morphisms) from $\mathrm{Th}_{a x}$ to theories (resp., axiom-preserving theory morphisms) from $\mathrm{Th}_{a x}^{\prime}$.
} 
by the components $\operatorname{Sign}_{0}$ and ax (and $\mathbf{S e n}_{0}$ in the case of theoroidal comorphisms between effective satisfiability subcalculi) of the underlying satisfiability calculi, disregarding the signatures and sentences outside that restriction.

In the same way we extended the notion of theoroidal comorphisms between institutions to satisfiability calculi (resp., satisfiability subcalculi / effective satisfiability subcalculi), we recall the notion of morphism between institutions and then extend it to satisfiability calculi which, in turn, can be analogously extended to morphisms of satisfiability subcalculi and morphisms of effective satisfiability subcalculi in a straightforward manner.

\section{Definition 4.5. (Institution morphism [5])}

Let $\mathbb{I}=\left\langle\right.$ Sign, Sen, Mod, $\left.\left\{\mid=_{\Sigma}\right\}_{\Sigma \in|\operatorname{Sign}|}\right\rangle$ and $\mathbb{I}^{\prime}=\left\langle\operatorname{Sign}^{\prime}, \mathbf{S e n}^{\prime}, \mathbf{M o d}^{\prime},\left\{||_{\Sigma}^{\prime}\right\}_{\Sigma \in\left|\operatorname{Sign}^{\prime}\right|}\right\rangle$ be institutions. Then, $\left\langle\nu^{S i g n}, \nu^{S e n}, \nu^{M o d}\right\rangle: \mathbb{I}^{\prime} \rightarrow \mathbb{I}$ is an institution morphism if and only if:

- $\nu^{\text {Sign }}: \operatorname{Sign}^{\prime} \rightarrow$ Sign is a functor,

- $\nu^{\text {Sen }}:$ Sen $\circ \nu^{\text {Sign }} \rightarrow$ Sen' $^{\prime}$, is a natural transformation (i.e. a natural family of functions $\left.\nu_{\Sigma^{\prime}}^{S e n}: \operatorname{Sen}\left(\nu^{\operatorname{Sign}}\left(\Sigma^{\prime}\right)\right) \rightarrow \operatorname{Sen}^{\prime}\left(\Sigma^{\prime}\right)\right)$, such that for each $\Sigma_{1}^{\prime}, \Sigma_{2}^{\prime} \in\left|\operatorname{Sign}^{\prime}\right|$ and $\sigma^{\prime}: \Sigma_{1}^{\prime} \rightarrow \Sigma_{2}^{\prime}$ morphism in Sign',
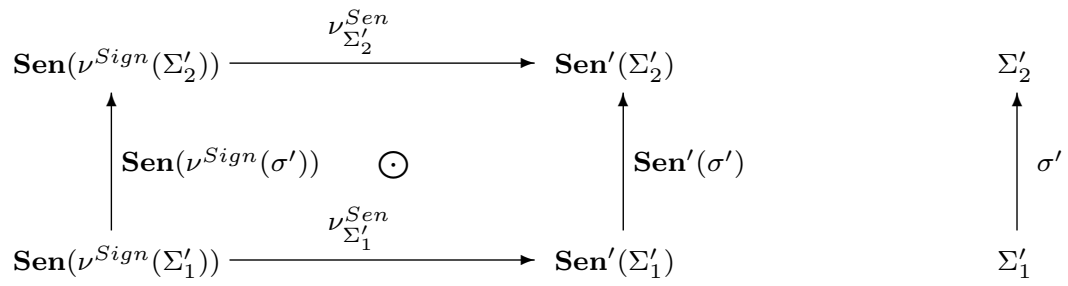

- $\nu^{M o d}: \operatorname{Mod}^{\prime} \rightarrow \operatorname{Mod} \circ\left(\nu^{S i g n}\right)^{\text {op }}$ is a natural transformation (i.e. the family of functors $\nu_{\Sigma^{\prime}}^{M o d}: \operatorname{Mod}^{\prime}\left(\Sigma^{\prime}\right) \rightarrow \operatorname{Mod}\left(\left(\nu^{S i g n}\right)^{\text {op }}\left(\Sigma^{\prime}\right)\right)$ is natural $)$, such that for each $\Sigma_{1}^{\prime}, \Sigma_{2}^{\prime} \in\left|\operatorname{Sign}^{\prime}\right|$ and $\sigma^{\prime}: \Sigma_{1}^{\prime} \rightarrow \Sigma_{2}^{\prime}$ morphism in Sign',
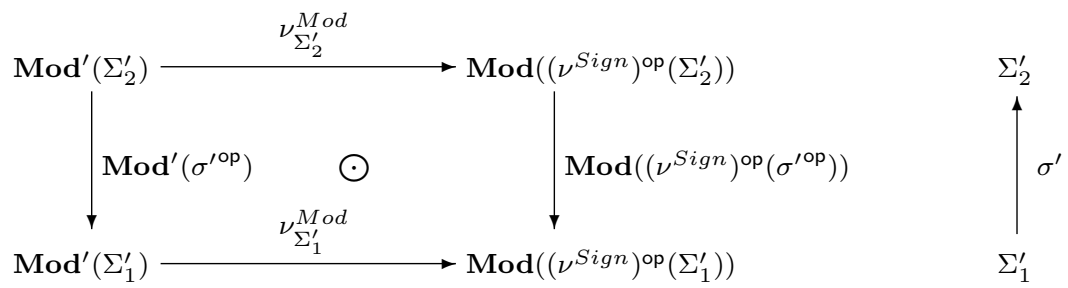

such that for any $\Sigma^{\prime} \in\left|\operatorname{Sign}^{\prime}\right|$, the function $\gamma_{\Sigma^{\prime}}^{\operatorname{Sen}}: \operatorname{Sen}\left(\nu^{\operatorname{Sign}}\left(\Sigma^{\prime}\right)\right) \rightarrow \operatorname{Sen}^{\prime}\left(\Sigma^{\prime}\right)$ and the functor $\nu_{\Sigma^{\prime}}^{M o d}: \operatorname{Mod}^{\prime}\left(\Sigma^{\prime}\right) \rightarrow \operatorname{Mod}\left(\left(\nu^{S i g n}\right)^{\mathrm{op}}\left(\Sigma^{\prime}\right)\right)$ preserves the following satisfaction condition: for any $\alpha \in \operatorname{Sen}\left(\nu^{\operatorname{Sign}}\left(\Sigma^{\prime}\right)\right)$ and $\mathcal{M} \in\left|\operatorname{Mod}\left(\Sigma^{\prime}\right)\right|$,

$$
\mathcal{M} \models \Sigma_{\Sigma^{\prime}} \nu_{\Sigma^{\prime}}^{S e n}(\alpha) \text { iff } \nu_{\Sigma^{\prime}}^{M o d}(\mathcal{M}) \models_{\nu^{\operatorname{Sign}}\left(\Sigma^{\prime}\right)} \alpha
$$


The extension of institution morphisms to theoroidal morphisms of institutions is obtained analogously to that of theoroidal comorphism. In [5], Tarlecki calls our attention to how, in contrast to institution comorphisms, institution morphisms capture how a "richer" institution (richer in terms of its model theory) is built on top of "poorer" ones and also shows in [5, Def. 7] that institutions together with institution morphisms form a category, named Ins, and in [5, Thm. 8] proves its completeness.

Institution morphisms also extend naturally to theoroidal morphisms between institutions by analogously considering $\nu^{S e n}$-sensible extensions of the functor mapping signatures. The following definition extends the notion of theoroidal morphisms of institutions to theoroidal morphisms of satisfiability calculi.

\section{Definition 4.6. (Theoroidal morphism of satisfiability calculi)}

Let $\mathbb{S}$ be the satisfiability calculus $\left\langle\right.$ Sign, Sen, Mod, $\left\{\models^{\Sigma}\right\}_{\Sigma \in|\operatorname{Sign}|}, \mathbf{M}$, Mods, $\left.\mu\right\rangle$ and $\mathbb{S}^{\prime}$ the satisfiability calculus $\left\langle\operatorname{Sign}^{\prime}, \mathbf{S e n}^{\prime}, \mathbf{M o d}^{\prime},\left\{\models^{{ }^{\Sigma}}\right\}_{\Sigma \in\left|\operatorname{Sign}^{\prime}\right|}, \mathbf{M}^{\prime}, \mathbf{M o d s}^{\prime}, \mu^{\prime}\right\rangle$.

Then, $\left\langle\nu^{T h}, \nu^{S e n}, \nu^{M o d}, \gamma\right\rangle: \mathbb{S}^{\prime} \rightarrow \mathbb{S}$ is a theoroidal morphism of satisfiability calculi if and only if:

- $\left\langle\nu^{T h}, \nu^{S e n}, \nu^{M o d}\right\rangle: \mathbb{I}^{\prime} \rightarrow \mathbb{I}$ is an theoroidal morphism of institutions, where $\nu^{T h}: \mathrm{Th} \rightarrow \mathrm{Th}^{\prime}$ is a $\nu^{\text {Sen }}$-sensible extension of $\nu^{\text {Sign }}$, and

- $\gamma:$ models $^{\prime} \rightarrow$ models $\circ \nu^{T h}$ is a natural transformation such that the following equality holds:
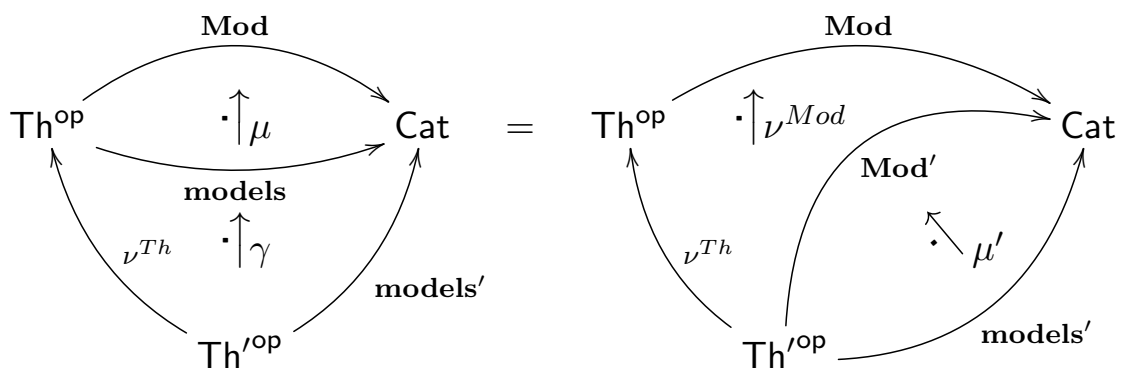

The reader should note that the sole change with respect to the definition of theoroidal institution comorphism is the direction of the arrow $\nu^{T h}$. This is a consequence of the change in the direction of the functor $\nu^{\text {Sign }}$ mapping signatures in the "richer" institution to signatures in the "poorer" one. As we mentioned before, the extension of this definition to morphisms between satisfiability subcalculi and effective satisfiability subcalculi provide results analogous to that in Definition 4.4 by taking into account the direction of the functor between signatures.

\subsection{Categories of satisfiability calculi, satisfiability subcalculi and effective satisfiabil- ity subcalculi}

The two functorial relations between satisfiability calculi presented above are relations between implementations of two (not necessarily different) satisfaction relations. The intuition behind them results analogous to that behind morphisms and comorphisms of institutions, where, in the first case, it corresponds to the observation of how an implementation of a richer satisfaction relation is built on top of 
a poorer one, and, in the second case, corresponds to how an implementation of a poorer satisfaction relation is represented in a richer one. In either case, it seems natural to investigate the structural properties of the class of satisfiability calculi together with these relations.

The following definitions and lemmas demonstrate that satisfiability calculi together with theoroidal morphisms between them form a category.

\section{Definition 4.7. (Identity theoroidal morphism of satisfiability calculi)}

Let the structure $\mathbb{S}=\left\langle\operatorname{Sign}\right.$, Sen, Mod, $\left.\left\{\mid=^{\Sigma}\right\}_{\Sigma \in|\operatorname{Sign}|}, \mathbf{M}, \mathbf{M o d s}, \mu\right\rangle$ be a satisfiability calculus and $\mathbb{I}$ its underlying institution. Then, the identity theoroidal morphism of satisfiability calculi $i d_{\mathbb{S}}: \mathbb{S} \rightarrow \mathbb{S}$ is formed by:

- The identity theoroidal morphism of institutions: $i d_{\mathbb{I}}: \mathbb{I} \rightarrow \mathbb{I}[2]$.

- The identity natural transformation $\gamma_{i d}:$ models $\dot{\rightarrow}$ models $\circ i d_{\mathbb{I}}^{T h}$, where $i d_{\mathbb{I}}^{T h}: \mathrm{Th}^{\mathbb{I}} \rightarrow \mathrm{Th}^{\mathbb{I}}$ maps every theory to itself.

On the other hand, the composition of two theoroidal morphisms of satisfiability calculi is constructed by composing their corresponding components, as detailed in the following definition.

\section{Definition 4.8. (Composition of theoroidal morphisms of satisfiability calculi)}

Given $\langle\nu, \gamma\rangle: \mathbb{S}^{\prime} \rightarrow \mathbb{S}$ and $\left\langle\nu^{\prime}, \gamma^{\prime}\right\rangle: \mathbb{S}^{\prime \prime} \rightarrow \mathbb{S}^{\prime}$ two theoroidal morphisms of satisfiability calculi. Their composition is defined componentwise as $\left\langle\nu \circ \nu^{\prime}, \widehat{\gamma}\right\rangle: \mathbb{S}^{\prime \prime} \rightarrow \mathbb{S}$ such that:

- $\nu \circ \nu^{\prime}: \mathbb{I}^{\prime \prime} \rightarrow \mathbb{I}$ is the composition of the theoroidal morphisms between the underlying institutions (see [5, Def. 7]) $)^{10}$, and

- the natural transformation $\widehat{\gamma}:$ models ${ }^{\prime \prime} \rightarrow \operatorname{models} \circ\left(\nu^{T h} \circ \nu^{\prime T h}\right)$ is formed by the components

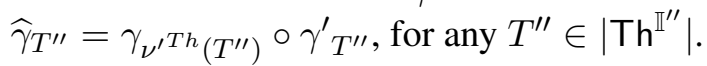

The following lemma shows the correctness of the definition above.

Lemma 4.9. Given two theoroidal morphisms of satisfiability calculi $\langle\nu, \gamma\rangle: \mathbb{S}^{\prime} \rightarrow \mathbb{S}$ and $\left\langle\nu^{\prime}, \gamma^{\prime}\right\rangle$ : $\mathbb{S}^{\prime \prime} \rightarrow \mathbb{S}^{\prime}$, then its composition $\langle\nu, \gamma\rangle \circ\left\langle\nu^{\prime}, \gamma^{\prime}\right\rangle: \mathbb{S}^{\prime \prime} \rightarrow \mathbb{S}$ is a theoroidal morphism of satisfiability calculi.

\section{Proof:}

The proof centres on proving the equality of the following 2-cells.

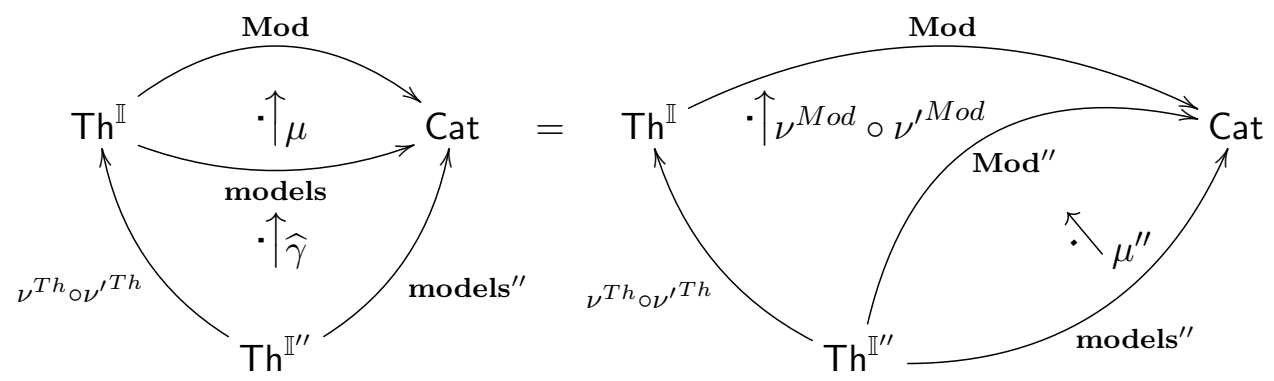

\footnotetext{
${ }^{10}$ Naturally, defining the composition of natural transformation does not only require a horizontal composition, denoted as " $\circ$ ", but also a vertical one acting as a multiplication of a natural transformation by a functor, which from now on will be denoted as ".".
} 
This follows from the following equalities (by a direct application of the definition of the composition): let $T^{\prime \prime} \in\left|\mathrm{Th}^{\mathbb{I}^{\prime \prime}}\right|$

$$
\begin{aligned}
& \mu_{\nu^{T h} \circ \nu^{\prime T h}\left(T^{\prime \prime}\right)} \circ \widehat{\gamma}_{T^{\prime \prime}}=\mu_{\nu^{T h} \circ \nu^{\prime T h}\left(T^{\prime \prime}\right)} \circ\left(\gamma_{\nu^{\prime T h}\left(T^{\prime \prime}\right)} \circ \gamma_{T^{\prime \prime}}^{\prime}\right) \\
& =\left(\mu_{\nu^{T h} \circ \nu^{\prime T h}\left(T^{\prime \prime}\right)} \circ \gamma_{\nu^{\prime T h}\left(T^{\prime \prime}\right)}\right) \circ \gamma_{T^{\prime \prime}}^{\prime} \\
& =\left(\mu_{\nu^{T h}\left(\nu^{\prime T h}\left(T^{\prime \prime}\right)\right)} \circ \gamma_{\nu^{\prime T h}\left(T^{\prime \prime}\right)}\right) \circ \gamma_{T^{\prime \prime}}^{\prime} \\
& \left.=\left(\nu_{\nu^{\prime T h}\left(T^{\prime \prime}\right)}^{M o d} \circ \mu_{\nu^{\prime T h}\left(T^{\prime \prime}\right)}^{\prime}\right) \circ \gamma_{T^{\prime \prime}}^{\prime} \quad \text { [ by 2-cell equality of }\langle\nu, \gamma\rangle\right] \\
& =\nu_{\nu^{\prime T h}\left(T^{\prime \prime}\right)}^{M o d} \circ\left(\mu_{\nu^{\prime T h}\left(T^{\prime \prime}\right)}^{\prime} \circ \gamma_{T^{\prime \prime}}^{\prime}\right) \\
& =\nu_{\nu^{\prime T h}\left(T^{\prime \prime}\right)}^{M o d} \circ\left(\nu_{T^{\prime \prime}}^{\prime M o d} \circ \mu_{T^{\prime \prime}}^{\prime \prime}\right) \quad \text { [ by 2-cell equality of }\left\langle\nu^{\prime}, \gamma^{\prime}\right\rangle \text { ] } \\
& =\left(\nu_{\nu^{\prime T h}\left(T^{\prime \prime}\right)}^{M o d} \circ \nu_{T^{\prime \prime}}^{M o d}\right) \circ \mu_{T^{\prime \prime}}^{\prime \prime}
\end{aligned}
$$

Theorem 4.10. The collection of satisfiability calculi together with the collection of theoroidal morphisms of satisfiability calculi form a category.

\section{Proof:}

First, consider two satisfiability calculi $\mathbb{S}=\left\langle\operatorname{Sign}\right.$, Sen, Mod, $\left.\left\{\left.\right|^{\Sigma}\right\}_{\Sigma \in|\operatorname{Sign}|}, \mathbf{M}, \mathbf{M o d s}, \mu\right\rangle$ and $\mathbb{S}^{\prime}=$ $\left\langle\operatorname{Sign}^{\prime}, \mathbf{S e n}^{\prime}, \mathbf{M o d}^{\prime},\left\{\mid=^{{ }^{\Sigma}}\right\}_{\Sigma \in\left|\operatorname{Sign}^{\prime}\right|}, \mathbf{M}^{\prime}, \mathbf{M o d s}^{\prime}, \mu^{\prime}\right\rangle$, and their respective underlying institutions $\mathbb{I}$ and $\mathbb{I}^{\prime}$. Consider any theoroidal morphism between the satisfiability calculi $\left\langle\nu^{T h}, \nu^{S e n}, \nu^{M o d}, \gamma\right\rangle$ : $\mathbb{S}^{\prime} \rightarrow \mathbb{S}$; then: $i d_{\mathbb{S}} \circ\left\langle\nu^{T h}, \nu^{S e n}, \nu^{M o d}, \gamma\right\rangle$ is defined as:

- $\left\langle i d_{\mathbb{I}^{\prime}}^{T h}, i d_{\mathbb{I}^{\prime}}^{S e n}, i d_{\mathbb{I}^{\prime}}^{M o d}\right\rangle \circ\left\langle\nu^{T h}, \nu^{S e n}, \nu^{M o d}\right\rangle$ which, by definition of composition of theoroidal morphisms between institutions, is $\left\langle\nu^{T h}, \nu^{S e n}, \nu^{M o d}\right\rangle$,

- let $T^{\prime} \in\left|\mathrm{Th}^{\mathbb{I}^{\prime}}\right|$ and $m \in\left|\operatorname{models}^{\prime}\left(T^{\prime}\right)\right|,\left(\gamma_{i d} \circ \gamma\right)_{T^{\prime}}(m)=\gamma_{i \nu^{T h}\left(T^{\prime}\right)}\left(\gamma_{T^{\prime}}(m)\right)=\gamma_{T^{\prime}}(m)$.

The proof for $\left\langle\nu^{T h}, \nu^{\text {Sen }}, \nu^{M o d}, \gamma\right\rangle \circ i d_{\mathbb{S}}$ is analogous to the previous one.

Now, let us prove that the composition of satisfiability calculi is associative. Consider satisfiability calculi $\mathbb{S}, \mathbb{S}^{\prime}, \mathbb{S}^{\prime \prime}$ and $\mathbb{S}^{\prime \prime \prime}$ together with theoroidal morphisms between satisfiability calculi $\left\langle\nu^{T h}, \nu^{S e n}, \nu^{M o d}, \gamma\right\rangle: \mathbb{S} \rightarrow \mathbb{S}^{\prime},\left\langle\nu^{\prime T h}, \nu^{\prime S e n}, \nu^{\prime M o d}, \gamma^{\prime}\right\rangle: \mathbb{S}^{\prime} \rightarrow \mathbb{S}^{\prime \prime}$ and $\left\langle\nu^{\prime \prime T h}, \nu^{\prime \prime S e n}, \nu^{\prime \prime M o d}, \gamma^{\prime \prime}\right\rangle:$ $\mathbb{S}^{\prime \prime} \rightarrow \mathbb{S}^{\prime \prime \prime}$. See [5, Def. 7] for a proof of the associativity of theoroidal morphisms of institutions. The remaining part the proof is as follows: let $T^{\prime \prime \prime} \in\left|\mathrm{Th}^{\mathbb{I}^{\prime \prime \prime}}\right|$

$$
\begin{aligned}
& \left(\left(\gamma \circ \gamma^{\prime}\right) \circ \gamma^{\prime \prime}\right)_{T^{\prime \prime \prime}}=\left(\gamma \circ \gamma^{\prime}\right)_{\nu^{\prime \prime T h}\left(T^{\prime \prime \prime}\right)} \circ \gamma_{T^{\prime \prime \prime}}^{\prime \prime}
\end{aligned}
$$

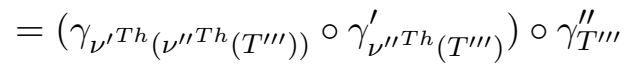

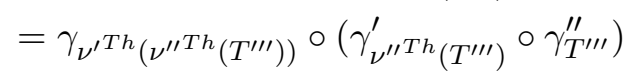

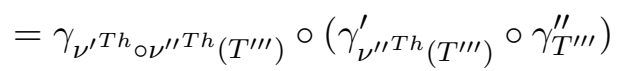

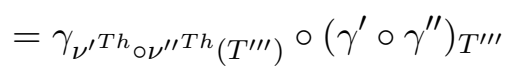

$$
\begin{aligned}
& =\left(\gamma \circ\left(\gamma^{\prime} \circ \gamma^{\prime \prime}\right)_{T^{\prime \prime \prime}}\right.
\end{aligned}
$$


We can consider extending the notion of identity theoroidal morphism of satisfiability calculi and composition of theoroidal morphisms between satisfiability calculi in order to consider the additional elements in the structure of a satisfiability subcalculi (resp., effective satisfiability subcalculi). The following two corollaries extend the previous result to the structure of satisfiability subcalculi (resp., effective satisfiability subcalculi) and morphisms between satisfiability subcalculi (resp., morphisms between effective satisfiability). They are straightforward extensions of Theorem 4.10.

Corollary 4.11. The collection of satisfiability subcalculi together with the collection of theoroidal morphisms between satisfiability subcalculi form a category.

Corollary 4.12. The collection of effective satisfiability subcalculi together with the collection of theoroidal morphisms between effective satisfiability subcalculi form a category.

The category formed by the collection of satisfiability calculi (resp., satisfiability subcalculi / effective satisfiability subcalculi) together with the collection of theoroidal morphisms between satisfiability calculi (resp., theoroidal morphisms between satisfiability subcalculi / theoroidal morphisms between effective satisfiability subcalculi) will be denoted as Sat (resp. Subsat / EffSubsat).

In [5, Sec. 5.2], Tarlecki points out the fact that limits in the category of institutions, with theoroidal morphisms between institutions, provide rudimentary mechanisms to combine different logical languages. Let us prove that completeness is preserved by the extension to category Sat.

Theorem 4.13. Sat is complete.

\section{Proof:}

Let $D:\langle I, E\rangle \rightarrow$ Sat be a diagram such that: 1) for all $i \in I, D(i)$ is the satisfiability calculus $\left\langle\operatorname{Sign}_{i}, \operatorname{Sen}_{i}, \mathbf{M o d}_{i},\left\{\models_{i}^{\Sigma}\right\}_{\Sigma \in\left|\operatorname{Sign}_{i}\right|}, \mathbf{M}_{i}, \mathbf{M o d s}_{i}, \mu_{i}\right\rangle$ is a satisfiability calculus, and 2) for all $e \in E$, $D(e): D(\operatorname{src}(e)) \rightarrow D(\operatorname{trg}(e))$ is a theoroidal morphism between satisfiability calculi of the form $\left\langle D(e)^{T h}, D(e)^{S e n}, D(e)^{M o d}, D(e)^{S a t}\right\rangle^{11}$.

First, we construct the limit object as follows:

- Since the category Ins is complete [33], we construct $\mathbb{I}=\left\langle\operatorname{Sign}, \operatorname{Sen}, \operatorname{Mod},\left\{\models^{\Sigma}\right\}_{\Sigma \in|\operatorname{Sign}|}\right\rangle$ as the limit object of the diagram that has as objects institutions $\mathbb{I}_{i}=\left\langle\operatorname{Sign}_{i}, \operatorname{Sen}_{i}, \mathbf{M o d}_{i},\left\{\models_{i}^{\Sigma}\right.\right.$ \}$\left._{\Sigma \in \operatorname{Sign}}\right\rangle$ and as arrows the institution morphisms $\left\{D(e): \mathbb{I}_{\operatorname{src}(e)} \rightarrow \mathbb{I}_{\operatorname{trg}(e)}\right\}_{e \in E}$.

- Let Struct ${ }_{S C}^{i}$ be the category used to capture the structure of canonical models for institution $D(i)$, for all $i \in I$. Then we define Struct $_{i}$ as the product $\mathrm{s}_{\mathrm{i}} \times$ Struct $_{S C}^{i}$, where $\mathrm{s}_{\mathrm{i}}$ is the category that contains a unique object $\left(s_{i}\right)$ and a unique arrow (the identity). Now, Struct $S C$ is the product in Cat of the Struct $_{i}$.

- Functor $\mathbf{M}$ is defined for each object as follows: $\mathbf{M}(t)_{i}=\left\langle s_{i}, \mathbf{M}_{i}\left(t_{i}\right)\right\rangle$ for each projection $i \in I$, and for each arrow $f: t \rightarrow t^{\prime}, \mathbf{M}(f)_{i}=\left\langle s_{i}, \mathbf{M}_{i}\left(f_{i}\right)\right\rangle$ for each $i \in I$, which is well defined.

\footnotetext{
${ }^{11} D(e)^{\text {Sat }}$ plays the role of the natural transformation $\gamma$ of Definition 4.6.
} 
- Functor Mods is defined by using a limit in Cat. For each object $c \in\left|\operatorname{Struct}_{S C}\right|$, we define $\operatorname{Mods}(c)$ as the limit of the diagram in Cat whose objects are $\mathbf{M o d s} \mathbf{s}_{i}\left(\mathbf{M}_{i}\left(t_{i}\right)\right)$ and arrows $D(e)_{t_{s r c(e)}^{S a t}}^{S: \text { models }}{ }^{\operatorname{trg}(e)}\left(D(e)^{\text {Th }}\left(t_{\operatorname{src}(e)}\right)\right) \rightarrow$ models $^{\operatorname{src}(e)}\left(t_{\operatorname{src}(e)}\right)$ in Cat. The naturality condition is guaranteed by the properties of limits.

- $\mu:$ models $\rightarrow \mathscr{P} \circ$ Mod is defined as follows. For each $t \in \mathrm{Th}_{0}, \mu_{t}: \operatorname{models}(t) \rightarrow$ $\mathscr{P} \circ \operatorname{Mod}(t)$ is constructed as a limit. Note that $\operatorname{models}(t)$ is a limit in Cat and similarly $\operatorname{Mod}(t)$ (see [59]); furthermore, we have a cone in Cat composed of arrows $D(e)_{i}^{M o d}{ }_{t_{i}} \circ \mu_{i t_{i}}$ : $\operatorname{models}_{i}\left(t_{i}\right) \rightarrow \mathscr{P} \circ \operatorname{Mod}(t)$, and noting that we have another cone composed of arrows $D(e)_{i}^{S a t}{ }_{t_{i}}: \operatorname{model}_{i}\left(t_{i}\right) \rightarrow \mathscr{P} \circ \operatorname{models}(t)$, by the universality of limits we should have a unique arrow (in Cat) $u_{t}: \operatorname{model}(t) \rightarrow \mathscr{P} \circ \operatorname{Mod}(t)$, so we define $\mu_{t}=u_{t}$; the naturality condition follows from the commutativity of the cones.

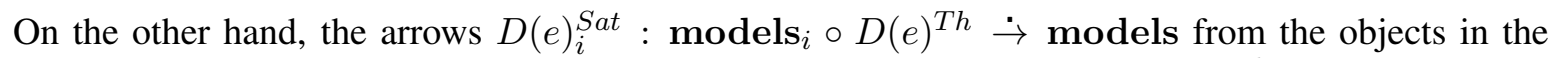
diagram to the limit object are defined by the injections $D(e)_{i_{t}}: \operatorname{model}_{i} \circ D(e)^{T h}(t) \rightarrow \operatorname{models}_{i}(t)$ in Cat. The equality of the 2-cells shown in the second condition of Definition 4.6 follows from the pointwise use of the validity of the equality of the 2-cells of each component. Finally, let us prove the universality of the given construction. Suppose that there exists another limit cone whose appex is $\mathbb{I}^{\prime}=\left\langle\operatorname{Sign}^{\prime}, \mathbf{S e n}^{\prime}, \mathbf{M o d}^{\prime},\left\{\models^{{ }^{\Sigma}}\right\}_{\Sigma \in\left|\operatorname{Sign}^{\prime}\right|}, \mathbf{M}^{\prime}, \mathbf{M o d s}^{\prime}, \mu^{\prime}\right\rangle$; since $\left\langle\operatorname{Sign}\right.$, Sen, Mod, $\left.\left\{\models^{\Sigma}\right\}_{\Sigma \in|\operatorname{Sign}|}\right\rangle$ is a limit in Ins we have a unique institution morphism $u: \mathbb{I}^{\prime} \rightarrow \mathbb{I}$, now this can be extended to a satisfiability mapping by considering the natural transformation $\gamma^{\prime}:$ models $\circ u^{T h} \dot{\rightarrow}$ models ${ }^{\prime}$ that has as components functors $\gamma_{t}^{\prime}:$ models $\circ u^{T h}(t) \rightarrow$ models ${ }^{\prime}(t)$ obtained by the universal property of products in Cat (as done in the definition of $\mu$ above), which are unique up to isomorphism.

The following corollaries follow from extending Theorem 4.13 to the categories Subsat and EffSubsat in order to cope with the functors connecting the categories of admissible signatures and the natural transformations connecting the functors identifying the admissible sentences of those signatures.

Corollary 4.14. Subsat is complete.

Corollary 4.15. EffSubsat is complete.

Analogous to the way in which we proved that satisfiability calculi together with theoroidal morphisms between them form a category, we can provide the definitions of identity theoroidal comorphism (analogous to Definition 4.7), composition of theoroidal comorphisms between satisfiability calculi (analogous to Definition 4.8) and prove that satisfiability calculi together with theoroidal comorphisms between them also form a category (from now on denoted as coSat).

Theorem 4.16. The collection of satisfiability calculi together with the collection of theoroidal comorphisms between satisfiability calculi form a category.

As in previous sections, the following two corollaries extend the previous result to satisfiability subcalculi together with theoroidal comorphisms between them and effective satisfiability subcalculi together with theoroidal comorphisms between them. 
Corollary 4.17. The collection of satisfiability subcalculi together with the collection of theoroidal comorphisms between satisfiability subcalculi form a category.

Corollary 4.18. The collection of effective satisfiability subcalculi together with the collection of theoroidal comorphisms between effective satisfiability subcalculi form a category.

Then again, we can prove the completeness of coSat (resp., coSubsat and coEffSubsat).

Theorem 4.19. coSat is complete.

\section{Proof:}

The proof is straightforward by extending the proof of [58, Thm. 5.3 $]^{12}$ and considering limits constructed dually to those appearing in the proof of Theorem 4.13.

Corollary 4.20. coSubsat is complete.

Corollary 4.21. coEffSubsat is complete.

\subsection{On cocompleteness and the representation of limits in Sat, Subsat and EffSub- sat}

Having proved the completeness of Sat (resp., Subsat and EffSubsat) and coSat (resp., coSubsat and coEffSubsat), its cocompleteness arises as a natural question. The fundamental importance of cocompleteness resides in the fact that it enables the modular construction of satisfiability calculi (resp., satisfiability subcalculi / effective satisfiability subcalculi) out of a diagram of satisfiability calculi (resp., satisfiability subcalculi / effective satisfiability subcalculi) and theoroidal morphisms (resp., comorphisms) between them, usually done by constructing colimits of those diagrams.

Cocompleteness of the categories Ins and colns have been more elusive and, in general, they do not hold. In [6, Ex. 4.10], Goguen and Roşu provide an example where the colimit cannot be constructed; providing also a negative answer to our previous questions. On the other hand, in [66, Thm. 25], Roşu proves that both the category of institutions over small signature categories together with institution morphisms, and institution comorphisms (denoted as SIns and coSIns, respectively) are cocomplete. We do not extend definitions and results to obtain a cocompleteness theorem for categories of satisfiability calculi over small signatures but we do not see any technical obstacle preventing the construction of proofs following the ideas used in Theorem 4.13.

In [5, Sec. 5.2] a relative answer to the general cocompleteness of Ins (resp., colns) was given in terms of the representation of institutions into a "universal" institution UII by means of comorphisms, provided that the category of signatures of $\mathbb{U} \mathbb{I}$ is cocomplete (see [59, Sec. 5.3] for details). Representation of theoroidal comorphisms were introduced by Tarlecki in [5], under the name representation map (of institutions representations) ${ }^{13}$, with the aim of establishing how, given an institution $\mathbb{I}^{\prime}$ built on

\footnotetext{
${ }^{12} \mathrm{~A}$ different proof of completeness of the category colns can be found in [66, Coro. 21].

${ }^{13}$ The reader should recall that in Tarlecki's work $[5,58,59]$, comorphisms of institutions where referred to by the name institution representations.
} 
top of an institution $\mathbb{I}$, witnessed by a morphism $\gamma: \mathbb{I}^{\prime} \rightarrow \mathbb{I} \in \mid$ Ins $\mid$, a comorphism $\rho^{\prime}: \mathbb{I}^{\prime} \rightarrow \mathbb{U I} \in \|$ UI $\|$ is built on top of a comorphism $\rho: \mathbb{I} \rightarrow \mathbb{U} \mathbb{I} \in\|\mathbb{U} \mathbb{I}\|$. From now on we assume a fixed but arbitrary "universal" institution $\mathbb{U I}=\left\langle\right.$ USign, USen, UMod, $\left.\left\{\left.\right|_{\Sigma}\right\}_{\Sigma \in \mid \text { USign } \mid}\right\rangle$ and a corresponding satisfiability calculus for $\mathbb{U I}, \mathbb{S} a t \mathbb{U I}=\left\langle\right.$ USign, USen, UMod, $\left.\left\{\left.\right|_{\Sigma}\right\}_{\Sigma \in \mid \text { USign } \mid}, \mathbf{U M}, \mathbf{U M o d s}, \mathbf{U} \mu\right\rangle$.

The following definition extends the notion of representations between comorphisms of institutions, presented in [5, Def. 16] to theoroidal comorphisms of institutions. It is obtained by considering that $\rho, \rho^{\prime}$ and $\gamma$ are now two theoroidal comorphisms and a theoroidal morphism, respectively. Thus, the second component of the representation is a natural transformation $\theta: \gamma^{T h} \circ \rho^{T h} \rightarrow \rho^{T h}$ and the properties it has to satisfy have to be extended to theories accordingly.

\section{Definition 4.22. (Representation of theoroidal comorphisms of institutions [5])}

Let $\mathbb{I}=\left\langle\right.$ Sign, Sen, Mod, $\left.\left\{\models_{\Sigma}\right\}_{\Sigma \in|\operatorname{Sign}|}\right\rangle, \mathbb{I}^{\prime}=\left\langle\operatorname{Sign}^{\prime}, \mathbf{S e n}^{\prime}, \mathbf{M o d}^{\prime},\left\{\models_{\Sigma}^{\prime}\right\}_{\Sigma \in\left|\operatorname{Sign}^{\prime}\right|}\right\rangle$ be institutions and, $\rho: \mathbb{I} \rightarrow \mathbb{U I}$ and $\rho^{\prime}: \mathbb{I}^{\prime} \rightarrow \mathbb{U I}$ their institution comorphisms to $\mathbb{U I}$. Then, $\langle\nu, \theta\rangle: \rho \rightarrow \rho^{\prime}$ is a representation of theoroidal comorphisms of institution if and only if:

- $\nu: \mathbb{I}^{\prime} \rightarrow \mathbb{I}$ is an theoroidal morphism of institutions, and

- $\theta: \rho^{T h} \circ \nu^{T h} \rightarrow \rho^{T h}$ is a natural transformation (i.e., a natural family of morphisms $\left\{\theta_{T^{\prime}}\right.$ : $\left.\left.\rho^{T h}\left(\nu^{T h}\left(T^{\prime}\right)\right) \rightarrow \rho^{\prime T h}\left(T^{\prime}\right)\right\}_{T^{\prime} \in\left|\mathrm{Th}^{\prime}\right|} \subseteq\|\mathrm{UTh}\|\right)$

such that:

- $\left(\rho^{\prime S e n} \cdot \operatorname{Sign}\right) \circ\left(\nu^{S e n} \cdot \operatorname{Sign}\right)=\left(\left(\operatorname{Sign} \circ \nu^{T h}\right) \cdot \rho^{S e n}\right) \circ(\theta \cdot \mathbf{U S e n})$, i.e. that for each $T^{\prime} \in\left|\mathrm{Th}^{\prime}\right|$ the following diagram commutes:

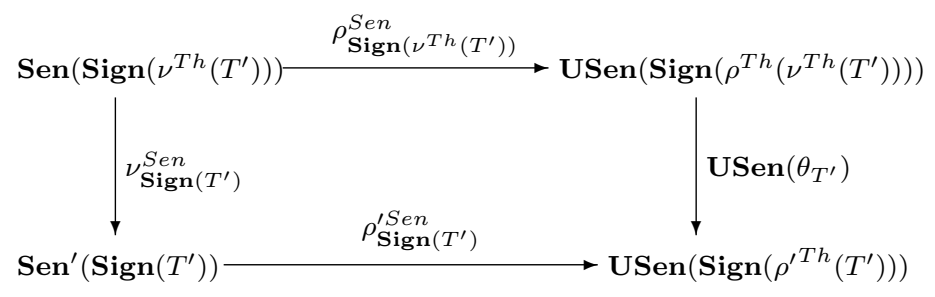

- $\left(\nu^{M o d} \cdot \mathbf{S i g n}\right) \circ\left(\rho^{\prime M o d} \cdot \mathbf{S i g n}\right)=\left(\theta^{\mathrm{op}} \cdot \mathbf{U M o d}\right) \circ\left(\left(\operatorname{Sign} \circ \nu^{T h^{\circ \mathrm{p}}}\right) \cdot \rho^{M o d}\right)^{14}$, i.e. that for each $T^{\prime} \in\left|\mathrm{Th}^{\prime}\right|$ the following diagram commutes:

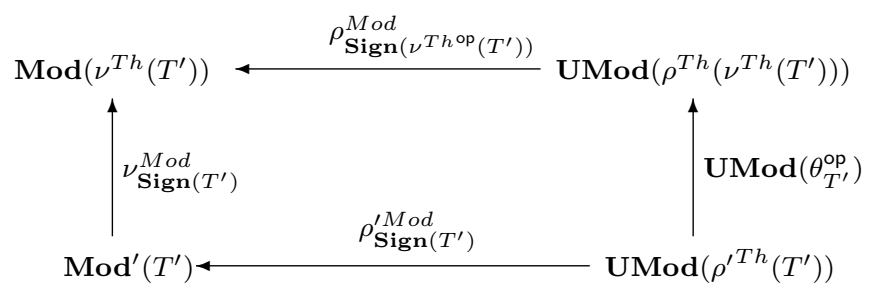

${ }^{14} \theta^{\mathrm{op}}: \rho^{\prime T h^{\mathrm{op}}} \rightarrow\left(\nu^{T h} \circ \rho^{T h}\right)^{\mathrm{op}}$ is the same natural transformation as $\theta: \nu^{T h} \circ \rho^{T h} \rightarrow \rho^{\prime T h}$ but considering its components to be the same functors between the opposite categories. 
As suggested by Tarlecki in his work, in the absence of a cocompleteness result for the category colns, a related result can be proved for a restriction of colns to those institutions representable in a given "universal" institution, witnessed by a family of theoroidal comorphisms between institutions. Let $\mathbb{U I}$ be an institution, then we denote by $\operatorname{lns}_{\mathbb{U I}}^{R e p r}$ the category whose objects are theoroidal comorphisms whose target institution is $\mathbb{U I}$ and whose morphisms are representations between these theoroidal comorphisms. Then, [5, Thm. 18] proves Ins $_{\mathbb{U I}}^{R e p r}$ cocompleteness providing the means for systematically representing combinations of logics of choice, taken from those representable in the "universal" institution.

Intuitively, a powerful logic with easy-to-understand and clear semantics, formal methods to assist the process of software specification and design, strongly based on rigorous definitions such as that of proof calculus (see Definition 2.8), and its variations of proof subcalculus and effective proof subcalculus (see [2, Defs. 14 and 16]) and/or satisfiability calculi, satisfiability subcalculi and effective satisfiability subcalculi (see Definitions 3.1, 3.4 and 3.6 respectively), may serve as a universal language into which the logical systems of interest can be interpreted and in which they can be put together. The next definition extends the notion of representation of theoroidal comorphisms of institutions to representations of theoroidal comorphisms of satisfiability calculi.

\section{Definition 4.23. (Representations of theoroidal comorphisms of satisfiability calculi)}

Let $\mathbb{S}$ be the satisfiability calculus $\left\langle\right.$ Sign, Sen, Mod, $\left\{\mid=^{\Sigma}\right\}_{\Sigma \in|\operatorname{Sign}|}, \mathbf{M}$, Mods, $\left.\mu\right\rangle$ and $\mathbb{S}^{\prime}$ the satisfiability calculus $\left\langle\operatorname{Sign}^{\prime}, \mathbf{S e n}^{\prime}, \mathbf{M o d}^{\prime},\left\{\mid=^{{ }^{\Sigma}}\right\}_{\Sigma \in\left|\operatorname{Sign}^{\prime}\right|}, \mathbf{M}^{\prime}, \mathbf{M o d} \mathbf{s}^{\prime}, \mu^{\prime}\right\rangle$, II and $\mathbb{I}^{\prime}$ their underlying institutions respectively, $\langle\nu, \theta\rangle: \mathbb{S}^{\prime} \rightarrow \mathbb{S}$ a theoroidal morphism between satisfiability calculi and $\langle\rho, \gamma\rangle: \mathbb{S} \rightarrow \mathbb{S} a t \mathbb{U I}$ and $\left\langle\rho^{\prime}, \gamma^{\prime}\right\rangle: \mathbb{S}^{\prime} \rightarrow \mathbb{S} a t \mathbb{U I}$ be theoroidal comorphisms of satisfiability calculi. Then, $\langle\langle\nu, \bar{\gamma}\rangle, \theta\rangle:\langle\rho, \gamma\rangle \rightarrow\left\langle\rho^{\prime}, \gamma^{\prime}\right\rangle$ is a representation of theoroidal comorphisms of satisfiability calculi if and only if:

- $\langle\nu, \theta\rangle: \rho \rightarrow \rho^{\prime}$ is a representation of theoroidal comorphisms of institutions,

such that:

- $\bar{\gamma} \circ \gamma^{\prime}=\left(\gamma \cdot \nu^{T h}\right) \circ\left(\right.$ Umodels $\left.\cdot \theta^{\text {op }}\right)$, i.e. that for each $T^{\prime} \in\left|\mathrm{Th}^{\prime}\right|$ the following diagram commutes:

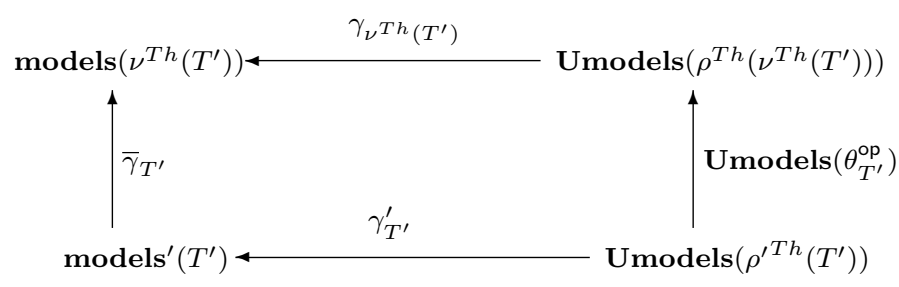

The notion of representations of theoroidal comorphisms of satisfiability calculi can be easily extended to define an analogous relation between theoroidal comorphisms between satisfiability subcalculi and effective satisfiability subcalculi by appropriately preserving the second condition of Definiton 4.4 . 


\section{Definition 4.24. (Identity representation of a theoroidal comorphism of sat. calculi)}

Let $\mathbb{S}=\left\langle\right.$ Sign, Sen, Mod, $\left\{\mid=^{\Sigma}\right\}_{\Sigma \in \mid \text { Sign } \mid}, \mathbf{M}$, Mods, $\left.\mu\right\rangle$ be a satisfiability calculus, $\mathbb{I}$ its underlying institution and $\langle\rho, \gamma\rangle: \mathbb{S} \rightarrow \mathbb{S} a t \mathbb{U I}$ a theoroidal comorphism of satisfiability calculi, where $\rho: \mathbb{I} \rightarrow \mathbb{U I}$ is a theoroidal comorphism of institutions. Then, the identity representation of a theoroidal comorphism of satisfiability calculi $\left\langle\left\langle i d_{\mathbb{I}}, \gamma_{i d}\right\rangle, \theta_{i d}\right\rangle:\langle\rho, \gamma\rangle \rightarrow\langle\rho, \gamma\rangle$ is formed by:

- The identity theoroidal morphism of satisfiability calculi: $\left\langle i d_{\mathbb{I}}, \gamma_{i d}\right\rangle: \mathbb{S}^{\prime} \rightarrow \mathbb{S}$ and

- The natural family of identity morphisms $\theta_{i d}: i d_{\mathbb{I}}^{T h} \circ \rho^{T h} \dot{\rightarrow} \rho^{T h}$.

\section{Definition 4.25. (Composition of representations of theoroidal comorphisms of sat. calculi)}

Let $\mathbb{S}, \mathbb{S}^{\prime}$ and $\mathbb{S}^{\prime \prime}$ be satisfiability calculi, consider $\langle\rho, \gamma\rangle: \mathbb{S} \rightarrow \mathbb{S} a t \mathbb{U I},\left\langle\rho^{\prime}, \gamma^{\prime}\right\rangle: \mathbb{S}^{\prime} \rightarrow \mathbb{S} a t \mathbb{U} \mathbb{I}^{\prime}$ and $\left\langle\rho^{\prime \prime}, \gamma^{\prime \prime}\right\rangle: \mathbb{S}^{\prime \prime} \rightarrow \mathbb{S} a t \mathbb{U I}$ be theoroidal comorphisms of satisfiability calculi and finally consider $\langle\langle\nu, \bar{\gamma}\rangle, \theta\rangle:\langle\rho, \gamma\rangle \rightarrow\left\langle\rho^{\prime}, \gamma^{\prime}\right\rangle$ and $\left\langle\left\langle\nu^{\prime}, \bar{\gamma}^{\prime}\right\rangle, \theta^{\prime}\right\rangle:\left\langle\rho^{\prime}, \gamma^{\prime}\right\rangle \rightarrow\left\langle\rho^{\prime \prime}, \gamma^{\prime \prime}\right\rangle$ be representations of theoroidal comorphisms of satisfiability calculi. Their composition is defined componentwise as $\left\langle\langle\nu, \bar{\gamma}\rangle \circ\left\langle\nu^{\prime}, \bar{\gamma}^{\prime}\right\rangle, \widehat{\theta}\right\rangle:\langle\rho, \gamma\rangle \rightarrow\left\langle\rho^{\prime \prime}, \gamma^{\prime \prime}\right\rangle$ such that:

- $\langle\nu, \bar{\gamma}\rangle \circ\left\langle\nu^{\prime}, \bar{\gamma}^{\prime}\right\rangle: \mathbb{S} \rightarrow \mathbb{S}^{\prime \prime}$ is the composition of theoroidal morphisms between the satisfiability calculi (see Def. 4.8), and

- the natural transformation $\widehat{\theta}: \rho \circ\left(\nu^{T h} \circ \nu^{\prime T h}\right) \dot{\rightarrow} \rho^{\prime \prime}$ is formed by the components $\widehat{\theta}_{T^{\prime \prime}}=$ $\theta_{\nu^{\prime T h}\left(T^{\prime \prime}\right)} \circ \theta^{\prime} T^{\prime \prime}$, for any $T^{\prime \prime} \in\left|\mathrm{Th}^{\mathbb{I}^{\prime \prime}}\right|$.

The reader should note that the composition of representations of theoroidal comorphisms of satisfiability calculi is defined analogously to the composition of representations of theoroidal comorphisms of institutions [5, Def. 17] as the only difference in the definition of the former, with respect to the latter, is the addition of conditions that the components have to satisfy.

Lemma 4.26. Let $\langle\langle\nu, \bar{\gamma}\rangle, \theta\rangle:\langle\rho, \gamma\rangle \rightarrow\left\langle\rho^{\prime}, \gamma^{\prime}\right\rangle$ and $\left\langle\left\langle\nu^{\prime}, \bar{\gamma}^{\prime}, \theta^{\prime}\right\rangle:\left\langle\rho^{\prime}, \gamma^{\prime}\right\rangle \rightarrow\left\langle\rho^{\prime \prime}, \gamma^{\prime \prime}\right\rangle\right.$ be two representations of theoroidal comorphisms of satisfiability calculi. Then, their composition $\langle\langle\nu, \bar{\gamma}\rangle, \theta\rangle \circ$ $\left\langle\left\langle\nu^{\prime}, \bar{\gamma}^{\prime}\right\rangle, \theta^{\prime}\right\rangle:\langle\rho, \gamma\rangle \rightarrow\left\langle\rho^{\prime \prime}, \gamma^{\prime \prime}\right\rangle$ is a representation of theoroidal comorphisms of satisfiability calculi.

\section{Proof:}

The proof follows by composing the diagrams derived from the hypothesis $\bar{\gamma} \circ \gamma^{\prime}=\left(\gamma \cdot \nu^{T h}\right) \circ$ $\left(\right.$ Umodels $\left.\cdot \theta^{\text {op }}\right)$ and $\bar{\gamma}^{\prime} \circ \gamma^{\prime \prime}=\left(\gamma^{\prime} \cdot \nu^{\prime T h}\right) \circ\left(\right.$ Umodels $\left.\cdot \theta^{\prime o p}\right)$.

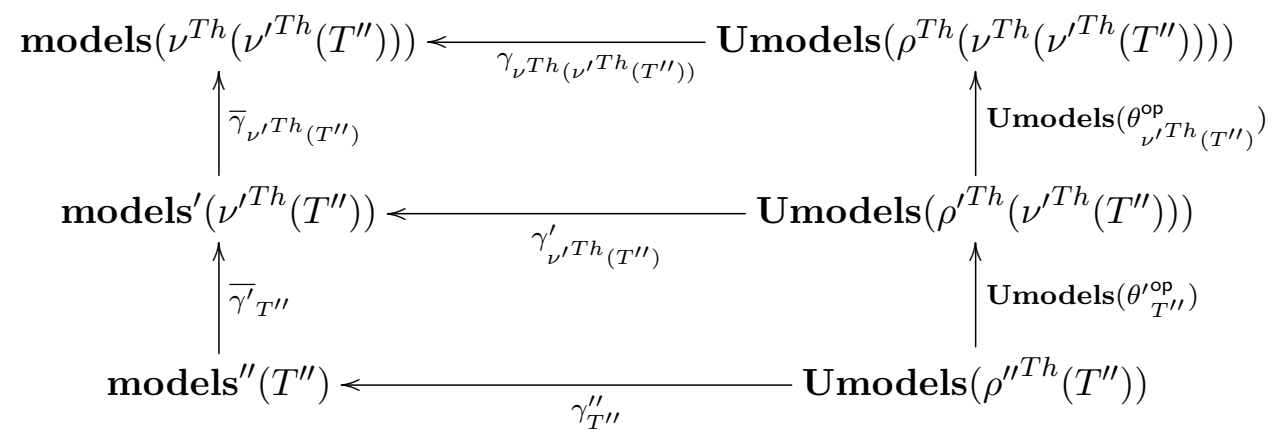


Then, $\left(\bar{\gamma}_{\nu^{\prime T h}\left(T^{\prime \prime}\right)} \circ \bar{\gamma}_{T^{\prime \prime}}^{\prime}\right) \circ \gamma^{\prime \prime}=\left(\gamma \cdot\left(\nu^{T h} \circ \nu^{\prime T h}\left(T^{\prime \prime}\right)\right)\right) \circ\left(\operatorname{Umodels}\left(\theta_{T^{\prime \prime}}^{\prime \text { op }}\right) \circ \operatorname{Umodels}\left(\theta_{\nu^{\prime T h}\left(T^{\prime \prime}\right)}^{\mathrm{op}}\right)\right)$, which is equivalent to $\left(\bar{\gamma}_{\nu^{\prime T h}\left(T^{\prime \prime}\right)} \circ \bar{\gamma}_{T^{\prime \prime}}^{\prime}\right) \circ \gamma^{\prime \prime}=\left(\gamma \cdot\left(\left(\nu \circ \nu^{\prime}\right)^{T h}\left(T^{\prime \prime}\right)\right)\right) \circ\left(\operatorname{Umodels}\left(\theta_{T^{\prime \prime}}^{\prime \circ} \circ \theta_{\nu^{\prime T h}\left(T^{\prime \prime}\right)}^{\mathrm{op}}\right)\right)$. Finally, by Definition 4.8, $\widehat{\gamma}_{T^{\prime \prime}}=\bar{\gamma}_{\nu^{\prime T h}\left(T^{\prime \prime}\right)} \circ \bar{\gamma}_{T^{\prime \prime}}^{\prime}$, for any $T^{\prime \prime} \in\left|\mathrm{Th}^{\mathbb{I}^{\prime \prime}}\right|$, by Definition 4.25, $\widehat{\theta}_{T^{\prime \prime}}=$ $\theta_{\nu^{\prime T h}\left(T^{\prime \prime}\right)} \circ \theta_{T^{\prime \prime}}^{\prime \prime}$, for any $T^{\prime \prime} \in\left|\mathrm{Th}^{\mathbb{I}^{\prime \prime}}\right|$. Thus, we obtain that $\widehat{\gamma} \circ \gamma^{\prime}=\left(\right.$ Umodels $\cdot\left(\widehat{\theta}^{\mathrm{op}}\right) \circ\left(\gamma \cdot\left(\nu^{\prime T h} \circ\right.\right.$ $\left.\left.\nu^{T h}\right)\right)$.

Theorem 4.27. Let $\mathbb{S} a t \mathbb{U I}$ be a satisfiability calculus for the "universal" institution $\mathbb{U} \mathbb{I}$ then, the collection of theoroidal comorphisms of satisfiability calculi to $\mathbb{S} a t \mathbb{U I}$ together with the class of representations of theoroidal comorphisms between satisfiability calculi is a category.

\section{Proof:}

The first part of the proof follows trivially by observing that the identity representation of theoroidal comorphisms is formed by an identity morphism, and two natural transformations whose components are all identity morphisms.

The proof of the associativity of the composition of representations of theoroidal comorphisms follows from the associativity of the composition of theoroidal morphisms of institutions, and of the components of both natural transformations.

From now on, given a satisfiability calculus $\mathbb{S} a t \mathbb{U I}$ for some "universal" institution $\mathbb{U} \mathbb{I}$, the category of theoroidal comorphisms from satisfiability calculi to $\mathbb{S} a t \mathbb{U I}$, as it was described in Theorem 4.27, will be denoted as Sat $\mathrm{S}_{\mathbb{S} a t \mathbb{U I}}^{\operatorname{Repr}}$. The previous definitions and results can easily be extended to prove that representations of theoroidal comorphisms of satisfiability subcalculi and effective satisfiability subcalculi also form categories, denoted as Subsat ${ }_{\mathbb{S} a t \mathbb{U I}}^{R e p r}$ and EffSubsat ${ }_{\mathbb{S} a t \mathbb{U}}^{R e p r}$, respectively.

The next theorem proves the relative cocompleteness of the Sat $t_{\mathbb{S a t} \mathbb{U I}}^{R e p r}$ by extending Tarlecki's proof of [5, Thm. 18] in order to consider the part of the theoroidal comorphisms relevant to satisfiability calculi.

Theorem 4.28. Let $\mathbb{S} a t \mathbb{U I}$ be a satisfiability calculus for the institution $\mathbb{U I}$. Then, if $\mathbf{S i g n}_{\mathbb{U I}}$ is cocomplete, Sat $\operatorname{Sat~} \mathrm{UI}_{\mathbb{I}}^{\text {is }}$ is cocomplete.

\section{Proof:}

Let $G=\langle N, E\rangle$ be a graph and $D: G \rightarrow$ Sat $_{\mathbb{S} a t \mathbb{U I}}^{\text {Repr }}$ be a diagram such that for all $i \in N, \mathbb{S}_{i}=$ $\left\langle\operatorname{Sign}_{i}, \operatorname{Sen}_{i}, \operatorname{Mod}_{i},\left\{\models_{i}^{\Sigma}\right\}_{\Sigma \in\left|\operatorname{Sign}_{i}\right|}, \mathbf{M}_{i}, \mathbf{M o d s}_{i}, \mu_{i}\right\rangle$ is a satisfiability calculus and $D(i)=\left\langle\rho_{i}, \gamma_{i}\right\rangle$ : $\mathbb{S}_{i} \rightarrow \mathbb{S} a t \mathbb{U I}$ is a theoroidal comorphism between satisfiability calculi, and for all $e \in E,\left\langle\nu_{e}, \bar{\gamma}_{e}\right\rangle$ : $\mathbb{S}_{s r c(e)} \rightarrow \mathbb{S}_{\operatorname{trg}(e)}$ is a theoroidal morphism between satisfiability calculi and $D(e)=\left\langle\left\langle\nu_{e}, \bar{\gamma}_{e}\right\rangle, \theta_{e}\right\rangle$ : $D(\operatorname{src}(e)) \rightarrow D(\operatorname{trg}(e))$ is a representation of theoroidal comorphisms between satisfiability calculi.

By Theorem 4.13, since Sat is complete, there exists $\mathbb{S} \in \mid$ Sat $\mid$ and $\left\{\left\langle\nu_{i}, \bar{\gamma}_{i}\right\rangle: \mathbb{S} \rightarrow \mathbb{S}_{i}\right\}_{i \in N} \subseteq$ $\|$ Sat $\|$ forming a limit for $D_{\text {Sat }}:\langle N, E\rangle \rightarrow$ Sat (i.e., the diagram formed by the satisfiability calculi in the source of the representations of comorphisms and the morphisms of satisfiability calculus of the representations of comorphisms of satisfiability calculi). By [5, Thm. 18], Ins $\mathrm{s}_{\mathbb{U I}}^{\text {Repr }}$ is cocomplete so there exists $\rho: \mathbb{I} \rightarrow \mathbb{U I} \in\left|\operatorname{lns} s_{\mathbb{U I}}^{R e p r}\right|$ and $\left\{\left\langle v_{i}^{\prime}, \theta_{i}^{\prime}\right\rangle: \rho_{i} \rightarrow \rho\right\}_{i \in N}$ forming a colimit for diagram $D_{\text {Ins }}^{R e p r}: G \rightarrow \operatorname{Ins}_{\mathbb{U I}}^{R e p r}$ (i.e., the diagram formed by the institutions, morphisms and comorphisms 
between institutions and representations of comorphisms between institutions underlying diagram $D$ ). These hypotheses can be seen in a more comprehensive way in the diagram shown below (solid arrows show a commutative triangle of diagram $D$ in Sat ${ }_{\mathbb{S} a t \mathbb{U}}^{R e p r}$, dashed arrows show the limit for $D_{\text {Sat }}$ in Sat and dotted arrows show the colimit for $D_{\operatorname{lns}}^{R e p r}$ in $\operatorname{Ins}_{\mathbb{U I}}^{R e p r}$ ).

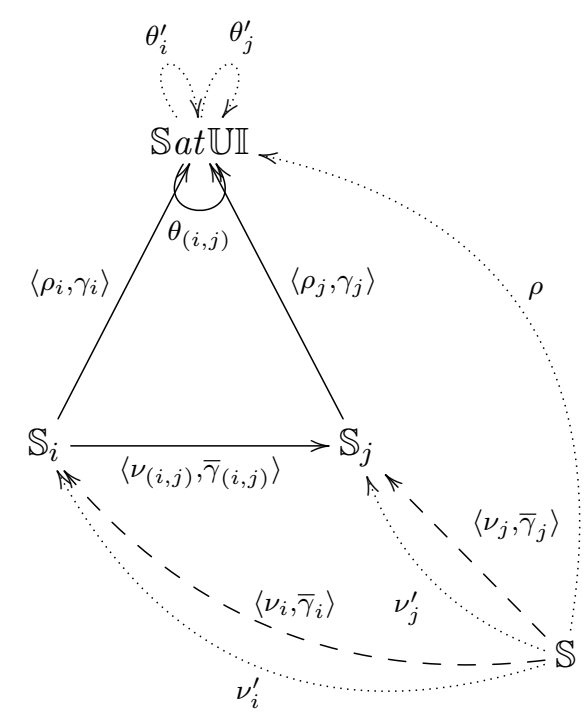

As $\mathbb{S}$ is the limit object for the diagram $D_{\text {Sat }}$, and by the way it is constructed (see Theorem 4.13 for details), for all $T \in\left|T h^{\mathbb{S}}\right|, \operatorname{models}^{\mathbb{S}}(T)$ constitute the limit object in Cat for the diagram formed by the corresponding categories of model structures models $\mathbb{S}_{i} \circ \nu_{i}^{T h}(T)$ and functors $\bar{\gamma}_{i}$ : $\operatorname{models}^{\mathbb{S}}(T) \rightarrow$ models $^{\mathbb{S}_{i}} \circ \nu_{i}^{T h}(T)$; then $\gamma_{T}:$ Umodels $\circ \rho^{T h}(T) \rightarrow \operatorname{models}^{\mathbb{S}}(T)$ is defined as the unique functor mapping each object $\mathcal{M} \in \mid$ Umodels $\circ \rho^{T h}(T) \mid$ to the unique object $\gamma_{T}(\mathcal{M}) \in\left|\operatorname{models}^{\mathbb{S}}(T)\right|$ such that for all $i \in N$,

$$
\left(\bar{\gamma}_{i}\right)_{T} \circ \gamma_{T}(\mathcal{M})=\gamma_{i_{i}^{T h}(T)} \circ \operatorname{Umodels}\left(\left(\theta_{i}^{\prime}\right)_{T}{ }^{\text {op }}\right)(\mathcal{M})
$$

This is well defined by the construction of $\mathbb{S}$. The definition for morphisms between canonical models is analogous. To prove that $\langle\rho, \gamma\rangle: \mathbb{S} \rightarrow \mathbb{S} a t \mathbb{U I}$ is a theoroidal comorphism of satisfiability calculim it remains to check the equivalence of Definition 4.2.

Let us prove that $\langle\rho, \gamma\rangle: \mathbb{S} \rightarrow \mathbb{S} a t \mathbb{U I}$ is a theoroidal comorphism of satisfiability calculi. That is, we have to prove:

$$
\mu_{T} \circ \gamma_{T}=\rho_{T}^{M o d} \circ \mu_{\rho^{T h}(T)}^{\mathbb{S a t} \mathbb{I}}
$$

for any $T \in \mathrm{Th}^{\mathbb{I}}$. First, note that since each $\left\langle\rho_{i}, \gamma_{i}\right\rangle: \mathbb{S}_{i} \rightarrow \mathbb{S} a t \mathbb{U I}$ is a theoroidal morphism between satisfiability calculi, we have that:

$$
\mu_{i T_{i}} \circ \gamma_{i T_{i}}=\rho_{i}^{M o d} T_{i} \circ \mu_{\rho_{i}^{T h}\left(T_{i}\right)}^{\mathbb{S a t \mathbb { I }}}
$$

Again, since $\mu$ and $\rho$ are defined by means of limits and colimits, respectively, and the definition of $\gamma$ is in terms of $\gamma_{i}$, by universality of limits we get the result. 
Also, note that, since $\left\{\left\langle\nu_{i}, \bar{\gamma}_{i}\right\rangle: \mathbb{S} \rightarrow \mathbb{S}_{i}\right\}_{i \in N}$ is a limit for $D_{\text {Sat }}^{R e p r}$ in Sat, and $\left\{\left\langle\nu_{i}^{\prime}, \theta_{i}^{\prime}\right\rangle: \rho_{i} \rightarrow\right.$ $\rho\}_{i \in N}$ is a colimit for $D_{\mathrm{Ins}}^{R e p r}$ in Ins $\mathrm{IUI}_{\mathbb{U I}}^{R e p r}$. Thus, by uniqueness of both limit and colimit, $\nu_{i}=\nu_{i}^{\prime}$ for all $i \in N$.

Now, let us prove that $\left\langle\left\langle\nu_{i}^{\prime}, \bar{\gamma}_{i}^{\prime}\right\rangle, \theta_{i}^{\prime}\right\rangle:\left\langle\rho_{i}, \gamma_{i}\right\rangle \rightarrow\langle\rho, \gamma\rangle$ are representation maps of theoroidal comorphisms between satisfiability calculi. That is, for each $i$ we must prove:

$$
\bar{\gamma}_{i} \circ \gamma=\left(\gamma_{i} \cdot \nu^{T h}\right) \circ\left(\mathbf{U m o d e l s} \cdot \theta_{i}^{\prime o p}\right)
$$

This straightforwardly follows from the definition of $\gamma$ given above.

Once again, given a "universal" institution $\mathbb{U I}$ and a satisfiability calculus $\mathbb{S} a t \mathbb{U I}$ for $\mathbb{U I}$, it is possible to extend Theorem 4.28 to the categories Subsat ${ }_{\mathbb{S a t} \mathbb{U I}}^{R e p r}$ and EffSubsat ${ }_{\mathbb{S a t} \mathbb{U}}^{R e p r}$ as shown in the following two corollaries.

Corollary 4.29. Let $\mathbb{S} a t \mathbb{U I}$ be a satisfiability subcalculus for the institution $\mathbb{U I}$. Then, if $\operatorname{Sign}_{\mathbb{U I}}$ is cocomplete, Subsat ${ }_{\mathbb{S} a t \mathbb{U I}}^{R e r}$ is cocomplete.

Corollary 4.30. Let $\mathbb{S} a t \mathbb{U I}$ be an effective satisfiability subcalculus for the institution $\mathbb{U I}$. Then, if Sign $_{\mathbb{U I}}$ is cocomplete, EffSubsat $\mathbb{S}_{\mathbb{S} a t \mathbb{U I}}^{\text {Repr }}$ is cocomplete.

\section{Conclusions and Further work}

Some decades ago, Meseguer [2] introduced the notion of proof calculus with the main aim of enriching institution theory. Broadly speaking, a proof calculus implements the deduction relation of an entailment system, a syntax driven definition of a logical system, in opposition to institutions [4], regarded as its abstract model theory. In this paper we introduced the notion of Satisfiability Calculus that might be thought of as the semantical counterpart of Meseguer's notion of proof calculus, providing the formal foundations for those proof systems resorting to model construction techniques to prove or disprove a given formula, serving as an implementation of the satisfiability relation of an institution. These techniques constitute an important stream of research in logic, in particular, in the areas of automatic software validation and verification. The abstract formalisation of these techniques provides a starting point for investigating their abstract and general properties. Furthermore, we believe that satisfiability calculus may provide the theoretical basis for the construction of heterogeneous semantic proof systems.

Several extensions of the framework presented here seem worth investigating. On the one hand, revisiting the definitions of proof calculus and satisfiability calculus, it is possible to note that the constraints imposed over some elements (e.g., the natural family of functors $\pi_{\langle\Sigma, \Gamma\rangle}:$ proofs $(\langle\Sigma, \Gamma\rangle) \rightarrow$ $\operatorname{Sen}(\langle\Sigma, \Gamma\rangle)$ and $\left.\mu_{\langle\Sigma, \Gamma\rangle}: \operatorname{model}(\langle\Sigma, \Gamma\rangle) \rightarrow \operatorname{Mod}(\langle\Sigma, \Gamma\rangle)\right)$ are, in some sense, too restrictive; working on generalisations of these concepts is part of our further work. In particular, it is worth noting that partial implementations of both the entailment relation and the satisfiability relation are gaining visibility in the software engineering community. On the other hand, there are many logical frameworks that have enjoyed increasing popularity in logic and software engineering which could be captured by weakening the relationship between entailment and the satisfiability relation. Examples, 
on the syntactic side, are the implementation of less expressive calculi with respect to an entailment, either for the sake of simplicity, as in the case of the finitary definition of the reflexive and transitive closure in Kleene algebras with tests [67] or, in the case of the implementation of rewriting tools like Maude [68], as a partial implementation of equational logic. Some examples on the semantic side are bounded model checkers and model finders for undecidable languages, usually available as software tools, such as Alloy [69] for relational logic, or the growing family of SMT-solvers [70] for languages including arithmetic. Clearly, allowing for partial implementations of entailment/satisfiability relations would enable us to capture the behaviours of some of the above mentioned logical tools. Removing this "restriction" implies allowing these partial implementations, in so far as they comply with behaving as a natural family of methods, which in this case implies that the monotonicity of deduction (respectively satisfaction) under change of notation has to be preserved. In addition, functorial relations between partial proof calculi (resp., satisfiability calculi) may provide a measure for how good the method is as an approximation of the ideal entailment relation (resp., satisfaction relation). We plan to explore these possibilities as future work.

\section{References}

[1] Goguen JA, Burstall RM. Introducing Institutions. In: Clarke EM, Kozen D (eds.), Proceedings of the Carnegie Mellon Workshop on Logic of Programs, volume 184 of Lecture Notes in Computer Science. Springer-Verlag, 1984 pp. 221-256.

[2] Meseguer J. General logics. In: Ebbinghaus HD, Fernandez-Prida J, Garrido M, Lascar D, Artalejo MR (eds.), Proceedings of the Logic Colloquium '87, volume 129. North Holland, Granada, Spain, 1989 pp. 275-329.

[3] Fiadeiro JL, Maibaum TSE. Generalising Interpretations between Theories in the context of $\pi$-Institutions. In: Burn G, Gay D, Ryan M (eds.), Proceedings of the First Imperial College Department of Computing Workshop on Theory and Formal Methods. Springer-Verlag, London, UK, 1993 pp. 126-147.

[4] Goguen JA, Burstall RM. Institutions: abstract model theory for specification and programming. Journal of the ACM, 1992. 39(1):95-146.

[5] Tarlecki A. Moving between logical systems. In: Haveraaen M, Owe O, Dahl OJ (eds.), Selected papers from the 11th Workshop on Specification of Abstract Data Types Joint with the 8th COMPASS Workshop on Recent Trends in Data Type Specification, volume 1130 of Lecture Notes in Computer Science. Springer-Verlag, 1996 pp. 478-502.

[6] Goguen JA, Roşu G. Institution Morphisms. Formal Aspects of Computing, 2002. 13(3-5):274-307.

[7] Sannella D, Tarlecki A. Specifications in an arbitrary institution. Information and computation, 1988. 76(2-3):165-210.

[8] Sannella D, Tarlecki A. Toward Formal Development of Programs from Algebraic Specifications: Implementations Revisited. Acta Informatica, 1988. 25(3):233-281. See also [71].

[9] Mossakowski T. Heterogeneous Development Graphs and Heterogeneous Borrowing. In: Nielsen M, Engberg U (eds.), Proceedings of the 5th International Conference on Foundations of Software Science and Computation Structures, Lecture Notes in Computer Science. Springer-Verlag, London, UK, 2002 pp. 326-341. 
[10] Mossakowski T, Tarlecki A. Heterogeneous Logical Environments for Distributed Specifications. In: Corradini A, Montanari U (eds.), Proceedings of 19th International Workshop in Algebraic Development Techniques, volume 5486 of Lecture Notes in Computer Science. Springer-Verlag, Pisa, Italy, 2009 pp. 266-289.

[11] Diaconescu R, Futatsugi K. Logical foundations of CafeOBJ. Theoretical Computer Science, 2002. 285(2):289-318.

[12] Diaconescu R. Grothendieck institutions. Applied Categorical Structures, 2002. 10(4):383-402.

[13] Diaconescu R (ed.). Institution-independent Model Theory, volume 2 of Studies in Universal Logic. Birkhäuser, 2008.

[14] Mossakowski T, Maeder C, Luttich K. The heterogeneous tool set, Hets. In: Grumberg O, Huth M (eds.), Proceedings of the 13th. International Conference on Tools and Algorithms for the Construction and Analysis of Systems (TACAS 2007), volume 4424 of Lecture Notes in Computer Science. SpringerVerlag, Braga, Portugal, 2007 pp. 519-522.

[15] Tarlecki A. Towards heterogeneous specifications. In: Gabbay D, de Rijke M (eds.), Frontiers of Combining Systems, volume 2 of Studies in Logic and Computation, pp. 337-360. Research Studies Press, 2000.

[16] Cengarle MV, Knapp A, Tarlecki A, Wirsing M. A heterogeneous approach to UML semantics. In: Degano P, DeNicola R, Meseguer J (eds.), Proceedings of Concurrency, graphs and models (Essays dedicated to Ugo Montanari on the occasion of his 65th. birthday), number 5065 in Lecture Notes in Computer Science. Springer-Verlag, Edinburgh, Scotland, 2008 pp. 383-402.

[17] Beth EW. The Foundations of Mathematics. North Holland, 1959.

[18] Beth EW. Semantic entailment and formal derivability. In: Hintikka J (ed.), The Philosophy of Mathematics, pp. 9-41. Oxford University Press, 1969. Reprinted from [72].

[19] Herbrand J. Investigation in Proof Theory. In: Goldfarb WD (ed.), Logical Writings, pp. 44-202. Harvard University Press, 1969. Translated to english from [73].

[20] Gentzen G. Investigation into logical deduction. In: Szabo ME (ed.), The Collected Papers of Gerhard Gentzen, pp. 68-131. North Holland, 1969. Translated to english from [74].

[21] Smullyan RM. First-order Logic. Dover Publishing, 1995.

[22] Robinson JA. A Machine-Oriented Logic Based on the Resolution Principle. Journal of the ACM, 1965. 12(1):23-41.

[23] Emerson EA. Temporal and Modal Logic. In: van Leeuwen J (ed.), Handbook of Theoretical Computer Science, volume B, pp. 995-1072. Elsevier, Amsterdam, 1990.

[24] Manna Z, Pnueli A. The Temporal Logic of Reactive and Concurrent Systems. Springer-Verlag, New York, NY, USA, 1992.

[25] Emerson EA, Clarke EM. Using branching time temporal logic to synthesize synchronization skeletons. Science of Computer Programming, 1982. 2(3):241-266.

[26] Attie PC. Synthesis of large dynamic concurrent programs from dynamic specifications. Formal Methods in System Design, 2016. 48(1):94-147. 
[27] Degiovanni R, Ricci N, Alrajeh D, Castro P, Aguirre NM. Goal-conflict Detection Based on Temporal Satisfiability Checking. In: Lo D, Apel S, Khurshid S (eds.), Proceedings of 31st IEEE/ACM International Conference on Automated Software Engineering. ACM Press, New York, NY, USA, 2016 pp. 507-518.

[28] Lopez Pombo CG, Castro P, Aguirre NM, Maibaum TSE. Satisfiability calculi: the semantic counterpart of proof calculi in general logics. In: Martí-Oliet N, Palomino Tarjuelo M (eds.), Proceedings of 21st International Workshop on Algebraic Development Techniques (WADT 2012) and IFIP International Federation for Information Processing (2013), volume 7841 of Lecture Notes in Computer Science. Springer-Verlag, Salamanca, Spain, 2013 pp. 195-211.

[29] McLane S. Categories for working mathematician. Graduate Texts in Mathematics. Springer-Verlag, Berlin, Germany, 1971.

[30] Fiadeiro JL. Categories for software engineering. Springer-Verlag, 2005.

[31] Cassano V, Lopez Pombo CG, Maibaum TSE. Entailment Systems for Default Reasoning. In: Martí-Oliet N, Palomino Tarjuelo M (eds.), Proceedings of 21st International Workshop on Algebraic Development Techniques (WADT 2012). Salamanca, Spain, 2012 pp. 28-30.

[32] Reiter R. A Logic for Default Reasoning. Artificial Intelligence, 1980. 13(1-2):81-132.

[33] Tarlecki A. Bits and pieces of the theory of institutions. In: Pitt DH, Abramsky S, Poigné A, Rydeheard DE (eds.), Proceedings of the Category Theory and Computer Programming, tutorial and workshop, volume 240 of Lecture Notes in Computer Science. Springer-Verlag, 1986 pp. 334-363.

[34] Lopez Pombo CG. Fork algebras as a tool for reasoning across heterogeneous specifications. Ph.D. thesis, Departamento de Computación, Facultad de Ciencias Exactas y Naturales, Universidad de Buenos Aires, 2007. Promotor: Marcelo F. Frias.

[35] Frias MF, Baum GA, Maibaum TSE. Interpretability of first-order dynamic logic in a relational calculus. In: de Swart H (ed.), Proceedings of the 6th. Conference on Relational Methods in Computer Science (RelMiCS) - TARSKI, volume 2561 of Lecture Notes in Computer Science. Springer-Verlag, Oisterwijk, The Netherlands, 2002 pp. 66-80.

[36] Haeberer AM, Veloso PA. Partial relations for program derivation: adequacy, inevitability and expressiveness. In: Proceedings of IFIP TC2 working conference on constructing programs from specifications. IFIP TC2: Software: Theory and Practice, North Holland, 1991 pp. 310-352.

[37] Frias MF. Fork algebras in algebra, logic and computer science, volume 2 of Advances in logic. World Scientific Publishing Co., Singapore, 2002.

[38] Fitting M. Tableau Methods of Proof for Modal Logics. Notre Dame Journal of Formal Logic, 1972. 13(2):237-247. Lehman College.

[39] Lewis CI. The Place of Intuition in Knowledge. Ph.D. thesis, Harvard University, 1910. Advisor: Tomas S. E. Maibaum.

[40] Lewis CI, Langford CH. Symbolic logic. The Century Co., 1932.

[41] Barcan RC. A Functional Calculus of First Order Based on Strict Implication. Journal of Symbolic Logic, 1946. 11(1):1-16.

[42] Barcan RC. The Deduction Theorem in a Functional Calculus of First Order Based on Strict Implication. Journal of Symbolic Logic, 1946. 11(4):115-118.

[43] Barcan RC. The Identity of Individuals in a Strict Functional Calculus of Second Order. Journal of Symbolic Logic, 1947. 12(1):12-15. 
[44] Kripke SA. Semantical Analysis of Modal Logic I. Normal Propositional Calculi. Zeitschrift fur mathematische Logik und Grundlagen der Mathematik, 1963. 9(56):67-96.

[45] Kripke SA. Semantical considerations on modal logic. Acta Philosophica Fennica, 1963. 16:83-94.

[46] Fitting M. First-Order Modal Tableaux. Journal of Automated Reasoning, 1988. 4(2):191-213.

[47] Fitting M, Mendelsohn RL. First-Order Modal Logic, volume 277 of Synthese Library. Springer Netherlands, 1998.

[48] Enderton HB. A mathematical introduction to logic. Academic Press, 1972.

[49] Schoenfield JR. Degrees of unsolvability. Number 2 in Mathematical studies. North Holland, 1971.

[50] Fitting M. Proof Methods for Modal and Intuitionistic Logics, volume 169 of Synthese Library. Springer Netherlands, 1983.

[51] Lopez Pombo CG, Castro P, Aguirre NM, Maibaum TSE. A Heterogeneous Characterisation of Component-Based System Design in a Categorical Setting. In: Ciobanu G, Ipate F (eds.), Proceedings of 11th International Colloquium Theoretical Aspects of Computing - ICTAC 2014, volume 8687 of Lecture Notes in Computer Science. Springer-Verlag, 2014 pp. 314-332.

[52] Castro P, Aguirre NM, Lopez Pombo CG, Maibaum TSE. A Categorical Approach to Structuring and Promoting Z Specifications. In: Pasareanu CS, Salaün G (eds.), Proceedings of 9th International Symposium Formal Aspects of Component Software - FACS 2012, volume 7684 of Lecture Notes in Computer Science. Springer-Verlag, 2013 pp. 73-91.

[53] Castro P, Aguirre NM, Lopez Pombo CG, Maibaum TSE. Categorical foundations for structured specifications in Z. Formal Aspects of Computing, 2015. 27(5-6):831-865.

[54] Borzyszkowski T. Logical systems for structured specifications. Theoretical Computer Science, 2002. 286:197-245.

[55] Mossakowski T, Tarlecki A. A Relatively Complete Calculus for Structured Heterogeneous Specifications. In: Muscholl A (ed.), Proceedings of 17th International Conference on Foundations of Software Science and Computation Structures (FOSSACS 2014), held as Part of the European Joint Conferences on Theory and Practice of Software, volume 8412 of Lecture Notes in Computer Science. Springer-Verlag, 2014 pp. 441-456.

[56] Lopez Pombo CG, Frias MF. Chapter 16: (Heterogeneous) Structured Specifications in Logics Without Interpolation, volume 17 of Outstanding Contributions to Logic, chapter 16. Springer International publishing, 2018.

[57] Castro P, Aguirre NM, Lopez Pombo CG, Maibaum TSE. Towards Managing Dynamic Reconfiguration of Software Systems in a Categorical Setting. In: Cavalcanti A, D'eharbe D, Gaudel MC, Woodcock J (eds.), Proceedings of Theoretical Aspects of Computing - ICTAC 2010, 7th International Colloquium, volume 6255 of Lecture Notes in Computer Science. Springer-Verlag, Natal, Rio Grande do Norte, Brazil, 2010 pp. 306-321.

[58] Tarlecki A. Structural properties of some categories of institutions, 1998. Revised version of its original version of 1996.

[59] Tarlecki A. Limits and colimits in some categories of institutions, 2006. Revised and extended version of [5].

[60] Blackburn P, de Rijke M, Venema Y. Modal logic. Number 53 in Cambridge Tracts in Theoretical Computer Science. Cambridge University Press, 2001. 
[61] Veloso PA, Haeberer AM, Frias MF. Fork algebras as algebras of logic. Abstracts of the Logic Colloquium '94, 1995. p. 127. Also in [75].

[62] Frias MF, Baum GA, Haeberer AM. Fork algebras in algebra, logic and computer science. Fundamenta Informaticae, 1997. 32:1-25.

[63] Jónnson B, Tarski A. Boolean algebra with operators, part I. American Journal of Mathematics, 1951. 73:891-939.

[64] Jónnson B, Tarski A. Boolean algebra with operators, part II. American Journal of Mathematics, 1952. 74:127-162.

[65] Frias MF, Lopez Pombo CG. Interpretability of first-order linear temporal logics in fork algebras. Journal of Logic and Algebraic Programming, 2006. 66(2):161-184.

[66] Roşu G. Kan Extensions of Institutions. Journal of Universal Computer Science, 1999. 5(8):482-493.

[67] Kozen D. Kleene algebra with tests. ACM Transactions on Programming Languages and Systems, 1997. 19(3):427-443.

[68] Clavel M, Eker S, Lincoln P, Meseguer J. Principles of Maude. In: Meseguer J (ed.), Proceedings of the 1st. International Workshop on Rewriting Logic and its Applications (WRLA'96), volume 4 of Electronic Notes in Theoretical Computer Science. Elsevier, 1996 pp. 65-89.

[69] Jackson D. Alloy: a lightweight object modelling notation. ACM Transactions on Software Engineering and Methodology, 2002. 11(2):256-290.

[70] de Moura LM, Bjørner N. Satisfiability modulo theories: introduction and applications. Communications of the ACM, 2011. 54(9):69-77.

[71] Sannella D, Tarlecki A. Toward formal development of programs from algebraic specifications: implementations revisited. Technical Report 17, Laboratory for foundations of computer science, The University of Edinburgh, 1986. Preliminar version of [8].

[72] Beth EW. Semantic entailment and formal derivability. Mededlingen van de Koninklijke Nederlandse Akademie van Wetenschappen, Afdeling Letterkunde, 1955. 18(13):309-342. Reprinted in [18].

[73] Herbrand J. Recherches sur la theorie de la demonstration. Ph.D. thesis, Université de Paris, 1930. English translation in [19].

[74] Gentzen G. Untersuchungen tiber das logische Schliessen. Mathematische Zeitschrijt, 1935. 39:176-210 and 405-431. English translation in [20].

[75] Veloso PA, Haeberer AM, Frias MF. Fork algebras as algebras of logic. Bulletin of Symbolic Logic, 1995. 1(2):265-266. Also in [61].

\section{A. Selected proofs}

In this section we will present detailed explanations, definitions and proofs of the results supporting the examples we presented in Sections 3 and 4. 


\section{A.1. Example 3.2: Tableau method for first-order predicate logic}

In Example 3.2 we presented the tableau method for first-order predicate logic and the intuitions for how it fits into the definition of a satisfiability calculus. In this section we will provide the formal definitions and the results proving it. Let $\mathbb{I}_{F O L}=\left\langle\operatorname{Sign}\right.$, Sen, Mod, $\left.\left\{\left.\right|^{\Sigma}\right\}_{\Sigma \in|\operatorname{Sign}|}\right\rangle$, the institution of first-order predicate logic.

Definition A.1. Let $\Sigma \in|\operatorname{Sign}|$ and $\Gamma \subseteq \operatorname{Sen}(\Sigma)$, we define $\operatorname{Str}^{\Sigma, \Gamma}=\langle\mathcal{O}, \mathcal{A}\rangle$ such that $\mathcal{O}=2^{\operatorname{Sen}(\Sigma)}$ and $\mathcal{A}=\left\{\alpha:\left\{A_{i}\right\}_{i \in \mathcal{I}} \rightarrow\left\{B_{j}\right\}_{j \in \mathcal{J}} \mid \alpha=\left\{\alpha_{j}\right\}_{j \in \mathcal{J}}\right\}$, where for all $j \in \mathcal{J}, \alpha_{j}$ is a branch in a tableau for $\Gamma \cup\left\{B_{j}\right\}$ with leaves $\Delta \subseteq\left\{A_{i}\right\}_{i \in \mathcal{I}} ; \Delta \models{ }^{\Sigma} \Gamma \cup\left\{B_{j}\right\}$ follows as a direct consequence of the definition.

Lemma A.2. Let $\Sigma \in|\operatorname{Sign}|$ and $\Gamma \subseteq \operatorname{Sen}(\Sigma)$, then $\operatorname{Str}^{\Sigma, \Gamma}$ defined as in Definition A.1 is a category.

\section{Proof:}

Let us prove that $\operatorname{Str}^{\Sigma, \Gamma}=\langle\mathcal{O}, \mathcal{A}\rangle$ is a category. For any set $\left\{A_{i}\right\}_{i \in \mathcal{I}} \in \mathcal{O}$, the identity is given by the collection of branches $\alpha_{i}:\left\{A_{i}\right\}$ (of length 1), i.e., no rule is applied.

Now, given $\alpha:\left\{A_{i}\right\}_{i \in \mathcal{I}} \rightarrow\left\{B_{j}\right\}_{j \in \mathcal{J}}, \beta:\left\{B_{j}\right\}_{j \in \mathcal{J}} \rightarrow\left\{C_{q}\right\}_{q \in \mathcal{Q}} \in \mathcal{A}$, their composition $\beta \circ \alpha=$ $\gamma$ is defined as follows: let $\left\{\alpha_{j}:\left\{B_{j}\right\} \rightarrow \cdots \rightarrow S \cup\left\{A_{i}\right\}_{i \in \mathcal{I}}\right\}_{j \in \mathcal{J}}$ and $\left\{\beta_{q}:\left\{C_{q}\right\} \rightarrow \cdots \rightarrow S^{\prime} \cup\right.$ $\left.\left\{B_{j}\right\}_{j \in \mathcal{J}}\right\}_{q \in \mathcal{Q}}$ be branches; then, $\left\{\gamma_{q}:\left\{C_{q}\right\} \rightarrow \cdots \rightarrow\left\{B_{j}\right\}_{j \in \mathcal{J}} \cup S^{\prime} \rightarrow \cdots \rightarrow\left\{A_{i}\right\}_{i \in \mathcal{I}} \cup S \cup S^{\prime}\right\}_{q \in \mathcal{Q}}$ is the branch obtained by extending each branch in $\beta$ with the corresponding branches in $\alpha$.

It remains to prove that $\circ$ has identities and is associative. Both proofs are straightforward by observing that $\circ$ is defined to be the concatenation of sequences of sets of formulae.

Lemma A.3. Let $\Sigma \in|\operatorname{Sign}|$ and $\Gamma \subseteq \operatorname{Sen}(\Sigma)$; then $\left\langle\operatorname{Str}^{\Sigma, \Gamma}, \cup, \emptyset\right\rangle$, where $\cup: \operatorname{Str}^{\Sigma, \Gamma} \times \operatorname{Str}^{\Sigma, \Gamma} \rightarrow$ $S t r^{\Sigma, \Gamma}$ is the typical bi-functor on sets and functions, and $\emptyset$ is the neutral element for $\cup$, is a strict monoidal category.

\section{Proof:}

Consider the bifunctor $\cup: \operatorname{Str}^{\Sigma, \Gamma} \times \operatorname{Str}^{\Sigma, \Gamma} \rightarrow \operatorname{Str}^{\Sigma, \Gamma}$ which behaves as follows: Given sets $A$ and $B$ $A \cup B$ is their union. Given a pair of arrows $\alpha: A \rightarrow B$ and $\beta: C \rightarrow D$, where $\alpha=\left\{\alpha_{i}\right\}_{i \in \mathcal{I}}$ and $\beta=\left\{\beta_{j}\right\}_{j \in \mathcal{J}}$, their union is $\alpha \cup \beta=\left\{\alpha_{i}\right\}_{i \in \mathcal{I}} \cup\left\{\beta_{j}\right\}_{j \in \mathcal{J}}$. Note that this functor is well defined: the union of the identities $i d_{\left\{A_{i}\right\}_{i \in \mathcal{I}}} \cup i d_{\left\{B_{j}\right\}_{j \in \mathcal{J}}}$ is a set of branches of length 1 and so is an identity too; and the composition is preserved, since it is built point wise. On the other hand, the identity object of the monoidal category is $\emptyset$ and the natural isomorphisms are given by the identity which trivially makes the associativity and identity diagrams commute.

Definition A.4. Struct $_{S C}$ is defined as $\langle\mathcal{O}, \mathcal{A}\rangle^{\text {op }}$ where $\mathcal{O}=\left\{\left\langle\operatorname{Str}^{\Sigma, \Gamma}, \cup, \emptyset\right\rangle|\Sigma \in| \operatorname{Sign} \mid \wedge \Gamma \subseteq\right.$ $\operatorname{Sen}(\Sigma)\}$, and $\mathcal{A}=\left\{\widehat{\sigma}:\left\langle\operatorname{Str}^{\Sigma, \Gamma}, \cup, \emptyset\right\rangle \rightarrow\left\langle\operatorname{Str}^{\Sigma^{\prime}, \Gamma^{\prime}}, \cup, \emptyset\right\rangle \mid \sigma:\langle\Sigma, \Gamma\rangle \rightarrow\left\langle\Sigma^{\prime}, \Gamma^{\prime}\right\rangle \in\|\operatorname{Th}\|\right\}$, the homomorphic extensions of the morphisms in $\|\mathrm{Th}\|$ to sets of formulae preserving the application of rules (i.e., the structure of the tableaux).

Lemma A.5. Let Struct $_{S C}$ be defined as in Def. A.4. Then, Struct $S C$ is a category. 


\section{Proof:}

First we prove that $\langle\mathcal{O}, \mathcal{A}\rangle$ where $\mathcal{O}=\left\{\left\langle\operatorname{Str}{ }^{\Sigma, \Gamma}, \cup, \emptyset\right\rangle|\Sigma \in| \operatorname{Sign} \mid \wedge \Gamma \subseteq \operatorname{Sen}(\Sigma)\right\}$, and $\mathcal{A}=\{\widehat{\sigma}$ : $\left.\left\langle S t r^{\Sigma, \Gamma}, \cup, \emptyset\right\rangle \rightarrow\left\langle\operatorname{Str}^{\Sigma^{\prime}, \Gamma^{\prime}}, \cup, \emptyset\right\rangle \mid \sigma:\langle\Sigma, \Gamma\rangle \rightarrow\left\langle\Sigma^{\prime}, \Gamma^{\prime}\right\rangle \in\|\mathrm{Th}\|\right\}$ is a category.

Morphisms $\widehat{\sigma} \in \mathcal{A}$ are the homomorphic extension of the morphisms $\sigma \in\|\mathrm{Th}\|$ to the structure of the tableaux, translating sets of formulae and preserving the application of the rules. Following this, the composition of $\widehat{\sigma_{1}}, \widehat{\sigma_{2}} \in \mathcal{A}$, the homomorphic extension of $\sigma_{1}, \sigma_{2} \in\|T h\|$, not only exists, but it is the homomorphic extension of the morphism $\sigma_{1} \circ \sigma_{2} \in\|\mathrm{Th}\|$. The associativity of the composition is also trivial to prove by considering that the morphisms are homomorphic extensions, and by the associativity of the composition of morphisms in Th. The identity morphism is the homomorphic extension of the identity morphism for the corresponding signature.

Then, as a direct consequence we obtain that Struct $_{S C}$ is a category.

Definition A.6. $\mathbf{M}:$ Th $^{\mathrm{op}} \rightarrow$ Struct $_{S C}$ is defined as $\mathbf{M}(\langle\Sigma, \Gamma\rangle)=\left\langle\operatorname{Str}^{\Sigma, \Gamma}, \cup, \emptyset\right\rangle$ and for any $\sigma:$ $\langle\Sigma, \Gamma\rangle \rightarrow\left\langle\Sigma^{\prime}, \Gamma^{\prime}\right\rangle \in\|\mathrm{Th}\|, \mathbf{M}\left(\sigma^{\text {op }}\right)=\widehat{\sigma}^{\text {op }}$ where $\widehat{\sigma}:\left\langle\operatorname{Str}^{\Sigma, \Gamma}, \cup, \emptyset\right\rangle \rightarrow\left\langle\operatorname{Str}^{\Sigma^{\prime}, \Gamma^{\prime}}, \cup, \emptyset\right\rangle$ is the homomorphic extension of $\sigma$ to the structures in $\left\langle\operatorname{Str}^{\Sigma, \Gamma}, \cup, \emptyset\right\rangle$.

Lemma A.7. Let $\mathbf{M}:$ Th $^{\text {op }} \rightarrow$ Struct $_{S C}$ be defined as in Definition A.6. Then $\mathbf{M}$ is a functor.

\section{Proof:}

Let $i d_{\langle\Sigma, \Gamma\rangle}:\langle\Sigma, \Gamma\rangle \rightarrow\langle\Sigma, \Gamma\rangle \in\|\mathrm{Th}\|$ be the identity morphism for $\langle\Sigma, \Gamma\rangle \in|\mathrm{Th}| . \mathbf{M}\left(i d_{\langle\Sigma, \Gamma\rangle}^{\text {op }}\right)=$ $i d_{\left\langle S t r^{\Sigma, \Gamma}, \cup, \emptyset\right\rangle}{ }^{\text {op }}$ because, by Def. A.4, $i d_{\left\langle S t r^{\Sigma, \Gamma}, \cup, \emptyset\right\rangle}$ is the homomorphic extension of $i d_{\langle\Sigma, \Gamma\rangle}$ to the structures in $\operatorname{Str}^{\Sigma, \Gamma}$.

Let $\sigma_{1}:\left\langle\Sigma_{1}, \Gamma_{1}\right\rangle \rightarrow\left\langle\Sigma_{2}, \Gamma_{2}\right\rangle, \sigma_{2}:\left\langle\Sigma_{2}, \Gamma_{2}\right\rangle \rightarrow\left\langle\Sigma_{3}, \Gamma_{3}\right\rangle \in\|\mathrm{Th}\|$; now, as composition of homomorphisms is a homomorphism, then $\mathbf{M}\left(\left(\sigma_{1} \circ \sigma_{2}\right)^{\mathrm{op}}\right)=\mathbf{M}\left(\sigma_{2}{ }^{\mathrm{op}} \circ \sigma_{1}{ }^{\mathrm{op}}\right)$ by definition of opposite category. Thus, it is the composition $\mathbf{M}\left(\sigma_{2}{ }^{\text {op }}\right) \circ \mathbf{M}\left(\sigma_{1}{ }^{\text {op }}\right)$.

Definition A.8. Mods : Struct $S C \rightarrow$ Cat is defined as:

- $\operatorname{Mods}\left(\left\langle S t r^{\Sigma, \Gamma}, \cup, \emptyset\right\rangle\right)=\langle\mathcal{O}, \mathcal{A}\rangle$ where:

$$
\begin{aligned}
& \mathcal{O}=\bigcup\left\{\langle\Sigma, \widetilde{\Delta}\rangle \mid\left(\exists \alpha: \Delta \rightarrow \emptyset \in\left|\operatorname{Str}^{\Sigma, \Gamma}\right|\right)(\widetilde{\Delta} \rightarrow \emptyset \in \alpha \wedge\right. \\
& \left.\left.\qquad\left(\forall \alpha^{\prime}: \Delta^{\prime} \rightarrow \Delta \in\left\|\operatorname{Str}^{\Sigma, \Gamma}\right\|\right)\left(\Delta^{\prime}=\Delta\right) \wedge \neg(\exists \varphi)(\{\neg \varphi, \varphi\} \subseteq \widetilde{\Delta})\right)\right\} \\
& \mathcal{A}=\left\{i d_{T}: T \rightarrow T \mid T \in \mathcal{O}\right\}, \text { and }
\end{aligned}
$$

- $\operatorname{Mods}\left(\widehat{\sigma}^{\mathrm{op}}\right)(\langle\Sigma, \delta\rangle)=\left\langle\Sigma^{\prime}, \operatorname{Sen}(\sigma)(\delta)\right\rangle$, for all $\sigma:\langle\Sigma, \Gamma\rangle \rightarrow\left\langle\Sigma^{\prime}, \Gamma^{\prime}\right\rangle \in\|\mathrm{Th}\|$.

Lemma A.9. Let Mods : Struct $S C \rightarrow$ Cat defined as in Definition A.8. Then, Mods is a functor.

\section{Proof:}

As for each theory $\left\langle\operatorname{Str}^{\Sigma, \Gamma}, \cup, \emptyset\right\rangle \in\left|\operatorname{Struct}_{S C}\right|, \operatorname{Mods}\left(\left\langle S \operatorname{tr}^{\Sigma, \Gamma}, \cup, \emptyset\right\rangle\right)$ is a discrete category containing theories whose models are also models of $\langle\Sigma, \Gamma\rangle$, thus the only property that must be proved is that for all $\widehat{\sigma}:\left\langle\operatorname{Str}^{\Sigma, \Gamma}, \cup, \emptyset\right\rangle \rightarrow\left\langle\operatorname{Str}^{\Sigma^{\prime}, \Gamma^{\prime}}, \cup, \emptyset\right\rangle \in\left\|\operatorname{Struct}_{S C}\right\|, o \in\left|\operatorname{Mods}\left(\left\langle\operatorname{Str}{ }^{\Sigma, \Gamma}, \cup, \emptyset\right\rangle\right)\right|$, $\operatorname{Mods}(\widehat{\sigma})(o) \in\left|\operatorname{Mods}\left(\left\langle S t r^{\Sigma^{\prime}, \Gamma^{\prime}}, \cup, \emptyset\right\rangle\right)\right|$. By definition, $\operatorname{Mods}(\widehat{\sigma})(\langle\Sigma, \widetilde{\Delta}\rangle)=\left\langle\Sigma^{\prime}, \operatorname{Sen}(\sigma)(\widetilde{\Delta})\right\rangle$. 
Observe that, as a consequence of the fact that $\widehat{\sigma}$ is the homomorphic extension of $\operatorname{Sen}(\sigma)$ to the tree-like structure of tableaux, the theory presentation obtained by applying $\operatorname{Mods}(\widehat{\sigma})$ to a particular element of $\operatorname{Mods}\left(\left\langle\operatorname{Str}^{\Sigma, \Gamma}, \cup, \emptyset\right\rangle\right)$ is a theory presentation whose set of axioms is a leaf of a branch of a tableau in $\left\langle S t r^{\Sigma^{\prime}, \Gamma^{\prime}}, \cup, \emptyset\right\rangle$.

Definition A.10. Let $\langle\Sigma, \Delta\rangle \in|\mathrm{Th}|$, we define $\mu_{\langle\Sigma, \Delta\rangle}: \operatorname{model} \mathbf{s}(\langle\Sigma, \Delta\rangle) \rightarrow \operatorname{Mod}_{F O L}(\langle\Sigma, \Delta\rangle)$ as for all $\langle\Sigma, \delta\rangle \in|\operatorname{models}(\langle\Sigma, \Delta\rangle)|, \mu_{\langle\Sigma, \Delta\rangle}(\langle\Sigma, \delta\rangle)=\operatorname{Mod}_{F O L}(\langle\Sigma, \delta\rangle)$.

Fact A.11. Let $\langle\Sigma, \Gamma\rangle \in|\mathrm{Th}|$ and $\mu_{\langle\Sigma, \Delta\rangle}: \operatorname{models}(\langle\Sigma, \Delta\rangle) \rightarrow \operatorname{Mod}_{F O L}(\langle\Sigma, \Delta\rangle)$ defined as in Def. A.10. Let $\Sigma \in\left|\operatorname{Sign}_{F O L}\right|$ and $\Gamma \subseteq \operatorname{Sen}_{F O L}(\Sigma)$, then $\mu_{\langle\Sigma, \Gamma\rangle}$ is a functor.

Lemma A.12. Let $\langle\Sigma, \Gamma\rangle \in|\mathrm{Th}|$ and $\mu_{\langle\Sigma, \Delta\rangle}: \operatorname{models}(\langle\Sigma, \Delta\rangle) \rightarrow \operatorname{Mod}_{F O L}(\langle\Sigma, \Delta\rangle)$ defined as in Definition A.10. Then, $\mu$ is a natural family of functors.

\section{Proof:}

Let $\langle\Sigma, \Gamma\rangle,\left\langle\Sigma^{\prime}, \Gamma^{\prime}\right\rangle \in|\mathrm{Th}|$ and $\sigma:\langle\Sigma, \Gamma\rangle \rightarrow\left\langle\Sigma^{\prime}, \Gamma^{\prime}\right\rangle \in\left|\mathrm{Th}_{0}\right|$. Then, the naturality condition for $\mu$ can be expressed in the following way:

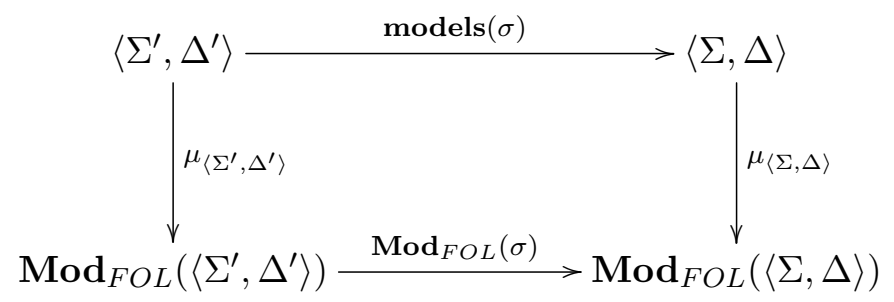

It is trivial to check that this condition holds by observing that canonical models are closed theories, thus behaving as theory presentations in Th.

Now, from Lemmas A.7, A.9, and A.12, and considering the hypothesis that $\mathbb{I}_{F O L}$ is an institution, the following corollary follows.

Corollary A.13. $\left\langle\right.$ Sign, Sen, Mod, $\left\{\left.\right|^{\Sigma}\right\}_{\Sigma \in|\operatorname{Sign}|}, \mathbf{M}$, Mods, $\left.\mu\right\rangle$ is a satisfiability calculus.

\section{A.2. Example 3.7: Effectiveness of the satisfiability subcalculus for finite presentations over the term-free restriction of first-order modal logic}

In Example 3.7 we presented an argument of how the satisfiability subcalculus of the term-free fragment of first-order modal logic of Example 3.5 fits the definition of an effective satisfiability subcalculus. In this section we will provide the formal definitions and the results proving it. Let us denote by $\mathbb{Q}=\left\langle\operatorname{Sign}\right.$, Sen, Mod, $\operatorname{Sign}_{0}$, $\left.\operatorname{Sen}_{0}, \mathbf{a x},\left\{=^{\Sigma}\right\}_{\Sigma \in|\operatorname{Sign}|}, \mathbf{M}, \mathbf{M o d s}, \mu\right\rangle$ a satisfiability subcalculus for first-order modal logic.

$\operatorname{Sign}_{0}$ is a complete subcategory of Sign so we assume $J: \operatorname{Sign}_{0} \rightarrow \operatorname{Sign}$ to be the functor that for all $\Sigma \in\left|\operatorname{Sign}_{0}\right|, J(\Sigma)=\Sigma$ and for all $\sigma \in\left\|\operatorname{Sign}_{0}\right\|, J(\sigma)=\sigma$. 
Definition A.14. Sen $_{0}$ is defined as the subfunctor of Sen resulting from restricting the latter to the objects and morphisms in $\operatorname{Sign}_{0}$.

Lemma A.15. $\operatorname{Sen}_{0}: \operatorname{Sign}_{0} \rightarrow$ Space is a functor.

\section{Proof:}

To prove that $\operatorname{Sen}_{0}: \operatorname{Sign}_{0} \rightarrow$ Space is a functor, we need to prove that: 1. given $\Sigma \in\left|\operatorname{Sign}_{0}\right|$, $\operatorname{Sen}_{0}(\Sigma)$ is a space, 2. given $\sigma: \Sigma \rightarrow \Sigma^{\prime} \in\left\|\operatorname{Sign}_{0}\right\|, \operatorname{Sen}_{0}(\sigma)$ is a total function between $\operatorname{Sen}_{0}(\Sigma)$ and $\operatorname{Sen}_{0}\left(\Sigma^{\prime}\right)$, and 3. $\operatorname{Sen}_{0}$ preserves identities and composition.

The first condition is trivial $\Sigma \in\left|\operatorname{Sign}_{0}\right|, \operatorname{Sen}_{0}(\Sigma)=\operatorname{Sen}(\Sigma)$ which is the infinite set of finite formulae recognised by the regular grammar presented in Example 3.5 for first-order modal logic. Thus, $\operatorname{Sen}_{0}(\Sigma)$ is a space. The second condition also results trivial because $\operatorname{Sen}_{0}(\sigma)$ is the homomorphic extension of $\sigma$ along the grammar mentioned before, so it is a function mapping formulae in space $\operatorname{Sen}_{0}(\Sigma)$ to formulae in space $\operatorname{Sen}_{0}\left(\Sigma^{\prime}\right)$. Finally, it is easy to observe that whenever $\operatorname{Sen}_{0}$ is applied to an identity morphism, the result is an identity function between formulae of the corresponding space. The preservation of composition also follows easily by checking that the composition of the homomorphic extensions of two morphisms results in the same function that the homomorphic extension of the composition of the morphisms.

Lemma A.16. Let $\mathcal{U}:$ Space $\rightarrow$ Set be the obvious forgetful functor projecting the underlying set of objects of the space and the total functions between them as morphisms; then $\mathcal{U} \circ \mathbf{S e n}_{0}=\operatorname{Sen} \circ J$.

\section{Proof:}

The proof follows by observing that: $1 . J: \operatorname{Sign}_{0} \hookrightarrow$ Sign is the identity inclusion functor, 2. the nature of $\mathcal{U}:$ Space $\rightarrow$ Set, and 3. Sen $_{0}$ is the subfunctor of Sen restricted to the objects and morphisms of $\operatorname{Sign}_{0}$

Let $\Sigma \in\left|\operatorname{Sign}_{0}\right|$, then, as $J$ is the identity inclusion functor, $J(\Sigma)=\Sigma$. Then, as $\Sigma \in\left|\operatorname{Sign}_{0}\right|$, $\operatorname{Sen}(\Sigma)=\mathcal{U}\left(\operatorname{Sen}_{0}(\Sigma)\right)$ because $\operatorname{Sen}_{0}$ is the subfunctor of Sen. The case of morphisms is analogous but considering sets, instead of functions.

Lemma A.17. ax : Sign $\rightarrow$ Space is a functor

\section{Proof:}

The proof is analogous to the one of Lemma A.15 but considering the extension of the functor $\mathbf{S e n}_{0}$, which operates on formulae, to finite sets of formulae.

Lemma A.18. Mods : Struct $S C \rightarrow$ Space is a functor.

\section{Proof:}

The proof is analogous to the one of Lemma A.17 but considering the extension of the functor ax, which operates on finite sets formulae, to finite tree-like structures whose nodes are finite sets of formulae. 


\section{A.3. Example 4.3: Mapping modal logic to first-order logic}

In Example 4.3 we presented the intuition behind the mapping from the tableau method for modal logic to the tableau method for first-order predicate logic. In this section we will provide the formal definitions and detailed proofs of the lemmas.

Let $\mathbb{S}_{F O L}$ be $\left\langle\operatorname{Sign}_{F O L}, \operatorname{Sen}_{F O L}, \mathbf{M o d}_{F O L},\left\{\mid={ }_{F O L}^{\Sigma}\right\}_{\Sigma \in\left|\operatorname{Sign}_{F O L}\right|}, \mathbf{M}_{F O L}, \mathbf{M o d s}_{F O L}, \mu_{F O L}\right\rangle$ and $\mathbb{S}_{K}$ be $\left\langle\operatorname{Sign}_{K}, \operatorname{Sen}_{K}, \mathbf{M o d}_{K},\left\{\mid=\sum_{K}^{\Sigma}\right\}_{\Sigma \in\left|\operatorname{Sign}_{K}\right|}, \mathbf{M}_{K}, \mathbf{M o d s}_{K}, \mu_{K}\right\rangle$ be the classic tableau methods for first-order predicate logic and modal logic, respectively, formalised as a satisfiability calculus.

Consider now the standard translation from modal logic to first-order logic presented in [60]. Therefore, the tuple $\left\langle\rho^{S i g n}, \rho^{S e n}, \rho^{M o d}\right\rangle$ is defined as follows:

Definition A.19. $\rho^{\operatorname{Sign}}: \operatorname{Sign}_{K} \rightarrow \operatorname{Sign}_{F O L}$ is defined as $\rho^{\operatorname{Sign}}\left(\left\langle\left\{p_{i}\right\}_{i \in \mathcal{I}}\right\rangle\right)=\left\langle R,\left\{p_{i}\right\}_{i \in \mathcal{I}}\right\rangle$ by mapping each propositional variable $p_{i}$, for all $i \in \mathcal{I}$, to a first-order unary logic predicate $p_{i}$, and adding a binary predicate $R$, and $\rho^{\operatorname{Sign}}\left(\sigma:\left\langle\left\{p_{i}\right\}_{i \in \mathcal{I}}\right\rangle \rightarrow\left\langle\left\{p_{i^{\prime}}^{\prime}\right\}_{i^{\prime} \in \mathcal{I}^{\prime}}\right\rangle\right)=\sigma^{\prime}:\left\langle R,\left\{p_{i}\right\}_{i \in \mathcal{I}}\right\rangle \rightarrow\left\langle R^{\prime},\left\{p_{i^{\prime}}^{\prime}\right\}_{i^{\prime} \in \mathcal{I}^{\prime}}\right\rangle$ mapping $R$ to $R^{\prime}$, and $p_{i}$ to $p_{i}^{\prime}$ for all $i \in \mathcal{I}$.

Lemma A.20. $\rho^{\text {Sign }}$ is a functor.

\section{Proof:}

To show that $\rho^{\text {Sign }}$ is a functor we have to prove that it preserves identity and composition. Consider a signature $\Sigma=\left\langle\left\{p_{i}\right\}_{i \in \mathcal{I}}\right\rangle$; the identity is just the mapping $\left\{p_{i} \mapsto p_{i}\right\}_{i \in \mathcal{I}}$. By Definition A.19 we obtain that $\left.\rho^{\Sigma}\left(\left\{p_{i} \mapsto p_{i}\right\}_{i \in \mathcal{I}}\right)=\{R \mapsto R\} \cup\left\{p_{i} \mapsto p_{i}\right\}_{i \in \mathcal{I}}\right\rangle$, thus yielding the identity for signature $\left\langle R,\left\{p_{i}\right\}_{i \in \mathcal{I}}\right\rangle$.

Let $\Sigma, \Sigma^{\prime}, \Sigma^{\prime \prime} \in|\operatorname{Sign}|$ and assume there are two morphisms $\sigma: \Sigma \rightarrow \Sigma^{\prime}, \sigma^{\prime}: \Sigma^{\prime} \rightarrow \Sigma^{\prime \prime} \in\left\|\mathrm{Th}_{0}\right\|$. Then $\rho^{\operatorname{Sign}}\left(\sigma \circ \sigma^{\prime}\right)=\rho^{\operatorname{Sign}}\left(\left\{p_{i} \mapsto p_{i}^{\prime \prime}\right\}\right)$, and therefore $\rho^{\operatorname{Sign}}\left(\sigma \circ \sigma^{\prime}\right)=\left\{R \mapsto R^{\prime \prime}\right\} \cup\left\{p_{i} \mapsto p_{i}^{\prime \prime}\right\}$. By definition of composition of functions $\left\{R \mapsto R^{\prime \prime}\right\} \cup\left\{p_{i} \mapsto p_{i}^{\prime \prime}\right\}=\left\{R \mapsto R^{\prime}\right\} \cup\left\{p_{i} \mapsto p_{i}^{\prime}\right\} \circ\left\{R^{\prime} \mapsto\right.$ $\left.R^{\prime \prime}\right\} \cup\left\{p_{i}^{\prime} \mapsto p_{i}^{\prime \prime}\right\}$, and consequently $\left\{R \mapsto R^{\prime \prime}\right\} \cup\left\{p_{i} \mapsto p_{i}^{\prime \prime}\right\}=\rho^{\operatorname{Sign}}(\sigma) \circ \rho^{\operatorname{Sign}}\left(\sigma^{\prime}\right)$.

\section{Definition A.21.}

Let $\left\langle\left\{p_{i}\right\}_{i \in \mathcal{I}}\right\rangle \in\left|\operatorname{Sign}_{K}\right|$. Then $\rho_{\left\langle\left\{p_{i}\right\}_{i \in \mathcal{I}}\right\rangle}^{\text {Sen }}: \operatorname{Sen}_{K}\left(\left\langle\left\{p_{i}\right\}_{i \in \mathcal{I}}\right\rangle\right) \rightarrow \rho^{\text {Sign }} \circ \operatorname{Sen}_{F O L}\left(\left\langle\left\{p_{i}\right\}_{i \in \mathcal{I}}\right\rangle\right)$ is defined recursively as $\rho_{\left\langle\left\{p_{i}\right\}_{i \in \mathcal{I}}\right\rangle}^{S e n}(\alpha)=T_{\left\langle\left\{p_{i}\right\}_{i \in \mathcal{I}}\right\rangle, x}(\alpha)$ where:

$$
\begin{aligned}
& T_{\left\langle\left\{p_{i}\right\}_{i \in \mathcal{I}\rangle, x}\right.}\left(p_{i}\right)=p_{i}^{\prime}(x) \text {, for all } i \in \mathcal{I} . \\
& T_{\left\langle\left\{p_{i}\right\}_{i \in \mathcal{I}}\right\rangle, x}(\neg \alpha)=\neg T_{\left\langle\left\{p_{i}\right\}_{i \in \mathcal{I}}\right\rangle, x}(\alpha) \\
& T_{\left\langle\left\{p_{i}\right\}_{i \in \mathcal{I}\rangle, x}\right.}(\alpha \vee \beta)=T_{\left\langle\left\{p_{i}\right\}_{i \in \mathcal{I}\rangle, x}\right.}(\alpha) \vee T_{\left\langle\left\{p_{i}\right\}_{i \in \mathcal{I}}, x\right.}(\beta)
\end{aligned}
$$

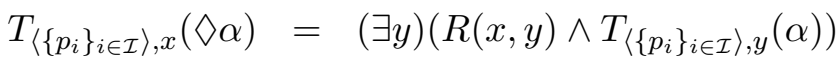

Fact A.22. Let $\left\langle\left\{p_{i}\right\}_{i \in \mathcal{I}}\right\rangle \in\left|\operatorname{Sign}_{K}\right|, \rho_{\left\langle\left\{p_{i}\right\}_{i \in \mathcal{I}}\right\rangle}^{S e n}$ is a function.

Lemma A.23. $\rho^{\text {Sen }}$ is a natural family of functions.

\section{Proof:}

To prove this lemma we must prove that the equality $\operatorname{Sen}_{K}(\sigma) \circ \rho_{\Sigma^{\prime}}^{S e n}=\rho_{\Sigma}^{S e n} \circ \operatorname{Sen}_{F O L}\left(\rho^{S i g n}(\sigma)\right)$ 
holds for every formula $\alpha \in\left|\mathrm{Th}^{K}\right|$. Notice that $\operatorname{Sen}_{K}(\sigma)$ and $\operatorname{Sen}_{F O L}\left(\rho^{\operatorname{Sign}}(\sigma)\right)$ only translate extra-logical symbols preserving the logical structure of the formulae because they are homomorphic extensions of the morphisms to the structure of the formulae induced by $\operatorname{Sen}_{K}$ and $\operatorname{Sen}_{F O L}$. On the other hand, the reader can see that two formulae that are $\alpha$-convertible yield, after the application of $\rho_{\Sigma}^{S e n}$ and $\rho_{\Sigma^{\prime}}^{S e n}, \alpha$-convertible formulae in the target category of sentences such that, by Definition A.19, preserving the mapping of extra-logical symbols.

Fact A.24. $\rho^{T h}$, the extension of $\rho^{\text {Sign }}$ defined as in Section 4, is $\rho^{S e n}$-sensible.

Definition A.25. Let $\left\langle\left\{p_{i}\right\}_{i \in \mathcal{I}}\right\rangle \in\left|\operatorname{Sign}_{K}\right|$, we define $\rho_{\left\langle\left\{p_{i}\right\}_{i \in \mathcal{I}}\right\rangle}^{M o d}: \rho^{\text {Sign }} \circ \operatorname{Mod}_{F O L}\left(\left\langle\left\{p_{i}\right\}_{i \in \mathcal{I}}\right\rangle\right) \rightarrow$ $\operatorname{Mod}_{K}\left(\left\langle\left\{p_{i}\right\}_{i \in \mathcal{I}}\right\rangle\right)$ as follows:

- for all $\mathcal{M}=\left\langle S, \bar{R},\left\{\bar{p}_{i}\right\}_{i \in \mathcal{I}}\right\rangle \in\left|\operatorname{Mod}_{F O L}\left(\left\langle R,\left\{p_{i}\right\}_{i \in \mathcal{I}}\right\rangle\right)\right|, \rho_{\left\langle\left\{p_{i}\right\}_{i \in \mathcal{I}}\right\rangle}^{M o d}(\mathcal{M})=\langle S, \bar{R}, L\rangle$, with $L\left(p_{i}\right)=\left\{s \in S \mid \overline{p_{i}}(s)\right\} .15$

- consider $\left\langle\left\{p_{i}\right\}_{i \in \mathcal{I}}\right\rangle \in\left|\operatorname{Sign}_{K}\right|$; then for all $h:\left\langle S_{1}, \overline{R_{1}},\left\{\overline{p_{1 i}}\right\}_{i \in \mathcal{I}}\right\rangle \rightarrow\left\langle S_{2}, \overline{R_{2}},\left\{\overline{p_{2 i}}\right\}_{i \in \mathcal{I}}\right\rangle \in$ $\left\|\operatorname{Mod}_{F O L}\left(\left\langle R,\left\{p_{i}\right\}_{i \in \mathcal{I}}\right\rangle\right)\right\|$, we define $\rho_{\left\langle\left\{p_{i}\right\}_{i \in \mathcal{I}}\right\rangle}^{M o d}(h)$ to be $\widehat{h}$, where $\widehat{h}\left(s_{1}\right)=s_{2}$ if and only if $h\left(s_{1}\right)=s_{2}$ for all $s_{1} \in S_{1}$.

Lemma A.26. Let $\left\langle\left\{p_{i}\right\}_{i \in \mathcal{I}}\right\rangle \in\left|\operatorname{Sign}_{K}\right|$, then $\rho_{\left\langle\left\{p_{i}\right\}_{i \in \mathcal{I}}\right\rangle}^{M o d}$ is a functor.

\section{Proof:}

It is trivial to prove that $\rho_{\left\langle\left\{p_{i}\right\}_{i \in \mathcal{I}\rangle}\right.}^{\text {Mod }}$ preserves identities by noting the definition of $\widehat{h}$ in terms of $h$.

The preservation of compositions follows by observing that, as predicates are mapped positionally, if we consider a pair of homomorphisms $h_{1}, h_{2} \in\left\|\operatorname{Mod}_{F O L}\left(\left\langle R,\left\{p_{i}\right\}_{i \in \mathcal{I}}\right\rangle\right)\right\|$, the resulting homomorphism $\rho_{\left\langle\left\{p_{i}\right\}_{i \in \mathcal{I}\rangle}\right.}^{M o d}\left(h_{1} \circ h_{2}\right) \in\left\|\operatorname{Mod}_{K}\left(\left\langle\left\{p_{i}\right\}_{i \in \mathcal{I}}\right\rangle\right)\right\|$ is exactly the homomorphism $\rho_{\left\langle\left\{p_{i}\right\}_{i \in \mathcal{I}\rangle}\right.}^{M o d}\left(h_{1}\right) \circ$ $\rho_{\left\langle\left\{p_{i}\right\}_{i \in \mathcal{I}}\right\rangle}^{M o d}\left(h_{2}\right) \in\left\|\operatorname{Mod}_{K}\left(\left\langle\left\{p_{i}\right\}_{i \in \mathcal{I}}\right\rangle\right)\right\|$.

Lemma A.27. $\rho^{M o d}$ is a natural family of functors (i.e., a natural transformation).

\section{Proof:}

Predicate symbols are mapped by resorting to the injective function $\sigma:\left\langle\left\{p_{i}\right\}_{i \in \mathcal{I}}\right\rangle \rightarrow\left\langle\left\{p_{i}^{\prime}\right\}_{i \in \mathcal{I}^{\prime}}\right\rangle \in$ $\left\|\operatorname{Sign}_{K}\right\|$ and, as $\rho_{\left\langle\left\{p_{i}\right\}_{i \in \mathcal{I}}\right\rangle}^{M o d}$ and $\rho_{\left\langle\left\{p_{i}^{\prime}\right\}_{i \in \mathcal{I}^{\prime}}\right\rangle}^{M o d}$ maps predicates interpreting the symbols in the $\rho^{\text {Sign }}$ translation of the signature positionally, the reduct operations $\operatorname{Mod}_{K}(\sigma)$ and $\operatorname{Mod}_{F O L}\left(\rho^{\operatorname{Sign}}(\sigma)\right)$ commute with $\rho_{\left\langle\left\{p_{i}\right\}_{i \in \mathcal{I}}\right\rangle}^{M o d}$ and $\rho_{\left\langle\left\{p_{i}^{\prime}\right\}_{i \in \mathcal{I}^{\prime}}\right\rangle}^{M o d}$, thus proving the naturality condition.

The next corollary follows from Lemmas A.20, A.23, and A.27.

Corollary A.28. $\left\langle\rho^{S i g n}, \rho^{S e n}, \rho^{M o d}\right\rangle$ is a comorphism of institutions.

\footnotetext{
${ }^{15}$ Notice that $\rho^{\text {Sign }}\left(\left\langle\left\{p_{i}\right\}_{i \in \mathcal{I}}\right\rangle\right)=\left\langle R,\left\{p_{i}\right\}_{i \in \mathcal{I}}\right\rangle$, where $\left\langle\left\{p_{i}\right\}_{i \in \mathcal{I}}\right\rangle \in\left|\operatorname{Sign}_{K}\right|$.
} 
The proof of this corollary ultimately relies on the correctness of the translation presented in [60]. Using this map we can define a mapping between the corresponding satisfiability calculi. Now, we have to prove that structures representing the tableaux for first-order predicate logic for properties resulting from the translation of modal logic properties can indeed be translated to modal logic tableaux for the original modal logic properties.

To continue with this example, it is important to recall that, whenever we consider set of formulae resulting from the application of function $\rho_{\left\langle\left\{p_{i}\right\}_{i \in \mathcal{I}}\right\rangle}^{S e n}$, the first-order predicate logic tableaux will have a particular shape because the application of rule $[\forall]$ (respectively $[\exists]$ ) is restricted to the formulae resulting from the application of the translation.

Fact A.29. Let $\left\langle\left\{p_{i}\right\}_{i \in \mathcal{I}}\right\rangle \in\left|\operatorname{Sign}_{K}\right|$; for all $\alpha \in\left|\operatorname{Sen}_{K}\left(\left\langle\left\{p_{i}\right\}_{i \in \mathcal{I}}\right\rangle\right)\right|$, if $\rho_{\left\langle\left\{p_{i}\right\}_{i \in \mathcal{I}}\right\rangle}^{\operatorname{Sen}}(\alpha)=\beta$, then any quantified sub-formula in $\beta$ is either of the form: $a)(\forall x)(R(y, x) \Longrightarrow \varphi(x))$, or $b)(\exists x)(R(y, x) \wedge$ $\varphi(x))$.

The next definition provides the means for obtaining modal logic tableaux from first-order predicate logic tableaux. In order to simplify the following definition, we will restrict ourselves to those first-order predicate logic tableaux in which, when the rule $[\forall]$ (respectively $[\exists]$ ) is applied, the rules $[\vee]$ and $[\neg]$ (respectively $[\wedge]$ ) are applied. Notice that this assumption does not limit the definitions and results in any way because any other legal tableau for the same set of formulae that does not satisfy this property can be reordered to satisfy it.

Definition A.30. We define $T$, a function translating first-order logic tableaux to modal logic tableaux, as follows ${ }^{16}$ :

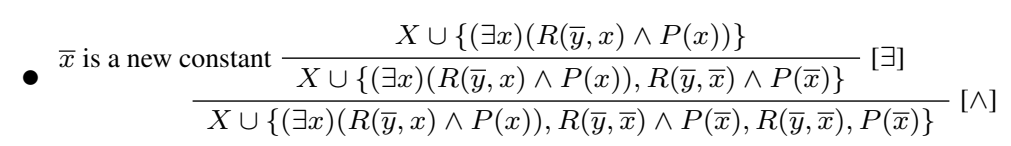

$$
\begin{aligned}
& \downarrow T \\
& \bar{x} \text { is a new label such that } R(\bar{y}, \bar{x}) \frac{X \cup\{\ell: \diamond P\}}{X \cup\{\ell: \diamond P, \bar{x}: P\}}[\diamond] \\
& \begin{array}{l|l}
\bar{x} \text { is a ground term } \frac{X \cup\{(\forall x)(\neg R(\bar{y}, x) \vee P(x))\}}{X \cup\{(\forall x)(\neg R(\bar{y}, x) \vee P(x)), \neg R(\bar{y}, \bar{x}) \vee P(\bar{x})\}}[\forall] \\
\hline \begin{array}{l|l}
X \cup\{(\forall x)(\neg R(\bar{y}, x) \vee P(x)), & X \cup\{(\forall x)(\neg R(\bar{y}, x) \vee P(x)), \\
\neg R(\bar{y}, \bar{x}) \vee P(\bar{x}), & \neg R(\bar{y}, \bar{x}) \vee P(\bar{x}), \\
\neg R(\bar{y}, \bar{x})\} & P(\bar{x})\}
\end{array} \\
\downarrow
\end{array} \\
& \bar{x} \text { is a label ocurring in } X \cup\{\ell\} \text { such that } R(\ell, \bar{x}) \frac{X \cup\{\ell: \square P\}}{X \cup\{\ell: \square P, \bar{x}: P\}}[\square]
\end{aligned}
$$

Then, the natural transformation $\gamma:$ models $^{\prime} \circ \rho^{T h} \dot{m}$ models is defined as follows.

\footnotetext{
${ }^{16}$ Notice that the translation of the rules for the propositional operators take care of the labelling by just preserving them.
} 
Definition A.31. Let $\left\langle\left\langle\left\{p_{i}\right\}_{i \in \mathcal{I}}\right\rangle, \Gamma\right\rangle \in\left|\mathrm{Th}^{K}\right|$; then

$$
\gamma_{\left\langle\left\langle\left\{p_{i}\right\}_{i \in \mathcal{I}}\right\rangle, \Gamma\right\rangle}: \operatorname{models}_{F O L} \circ \rho^{T h}\left(\left\langle\left\langle\left\{p_{i}\right\}_{i \in \mathcal{I}}\right\rangle, \Gamma\right\rangle\right) \rightarrow \operatorname{models}_{K}\left(\left\langle\left\langle\left\{p_{i}\right\}_{i \in \mathcal{I}}\right\rangle, \Gamma\right\rangle\right)
$$

is defined as:

$$
\gamma_{\left\langle\left\langle\left\{p_{i}\right\}_{i \in \mathcal{I}}\right\rangle, \Gamma\right\rangle}\left(\left\langle\left\langle R,\left\{p_{i}\right\}_{i \in \mathcal{I}}\right\rangle, \Delta\right\rangle\right)=\left\langle\left\langle\left\{p_{i}\right\}_{i \in \mathcal{I}}\right\rangle,\left\{\varphi \in\left|\operatorname{Sen}_{K}\left(\left\langle\left\{p_{i}\right\}_{i \in \mathcal{I}}\right\rangle\right)\right| \mid \rho_{\left\langle\left\{p_{i}\right\}_{i \in \mathcal{I}}\right\rangle}^{S e n}(\varphi) \in \Delta\right\}\right\rangle
$$

where $\rho^{T h}\left(\left\langle\left\langle\left\{p_{i}\right\}_{i \in \mathcal{I}}\right\rangle, \Gamma\right\rangle\right)=\left\langle\left\langle R,\left\{p_{i}\right\}_{i \in \mathcal{I}}\right\rangle, \Delta\right\rangle$.

Lemma A.32. Let $\left\langle\left\langle\left\{p_{i}\right\}_{i \in \mathcal{I}}\right\rangle, \Gamma\right\rangle \in\left|\mathrm{Th}^{K}\right|$, then $\gamma_{\left\langle\left\langle\left\{p_{i}\right\}_{i \in \mathcal{I}}\right\rangle, \Gamma\right\rangle}$ is a functor.

\section{Proof:}

This lemma follows trivially by observing that the categories obtained by applying models $\mathbf{s}_{F O L}$ and models $\mathbf{m}_{K}$ are discrete, and that given $\left\langle\left\langle R,\left\{p_{i}\right\}_{i \in \mathcal{I}}\right\rangle, \Delta\right\rangle \in\left|\mathrm{Th}^{F O L}\right|$, the theory presentation $\left\langle\left\langle\left\{p_{i}\right\}_{i \in \mathcal{I}}\right\rangle,\left\{\alpha \in\left|\operatorname{Sen}_{K}\left(\left\langle\left\{p_{i}\right\}_{i \in \mathcal{I}}\right\rangle\right)\right| \mid \rho_{\left\langle\left\{p_{i}\right\}_{i \in \mathcal{I}}\right\rangle}^{S e n}(\alpha) \in \Delta\right\}\right\rangle$ is the canonical model obtained from a branch of the modal logic tableau resulting from the application of $T$ to the tableau from which $\left\langle\left\langle R,\left\{p_{i}\right\}_{i \in \mathcal{I}}\right\rangle, \Delta\right\rangle$ was obtained.

Lemma A.33. $\left\{\gamma_{T}: \text { models } F O L \circ \rho^{T h}(T) \rightarrow \operatorname{model}_{K}(T)\right\}_{T \in\left|T h^{K}\right|}$ is a natural family of functors (i.e., a natural transformation).

\section{Proof:}

Let $\sigma:\left\langle\left\{p_{i}\right\}_{i \in \mathcal{I}}\right\rangle \rightarrow\left\langle\left\{p_{i}^{\prime}\right\}_{i \in \mathcal{I}^{\prime}}\right\rangle \in\left\|\operatorname{Sign}_{K}\right\|$ and $\varphi:\left\langle R,\left\{p_{i}\right\}_{i \in \mathcal{I}}\right\rangle \rightarrow\left\langle R^{\prime},\left\{p_{i}^{\prime}\right\}_{i \in \mathcal{I}^{\prime}}\right\rangle \in\left\|\operatorname{Sign}_{F O L}\right\|$ such that $\rho^{\operatorname{Sign}}(\sigma)=\varphi$ then, the naturality condition for $\gamma$ can be drawn as follows:

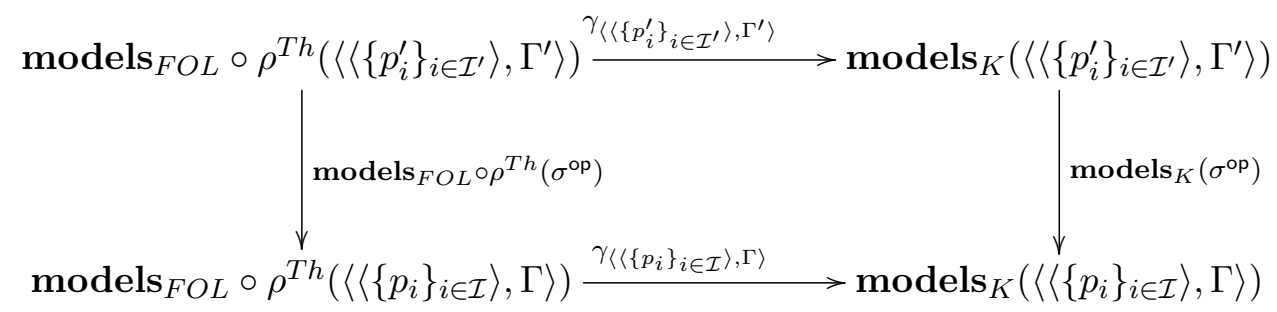

Let $\left\langle\left\langle R^{\prime},\left\{p_{i}^{\prime}\right\}_{i \in \mathcal{I}^{\prime}}\right\rangle, \Delta\right\rangle \in\left|\operatorname{model}_{F O L} \circ \rho^{T h}\left(\left\langle\left\langle\left\{p_{i}^{\prime}\right\}_{i \in \mathcal{I}^{\prime}}\right\rangle, \Gamma\right\rangle\right)\right|$, then, by resorting to Definition A.31, we get that

$$
\gamma_{\left\langle\left\langle\left\{p_{i}^{\prime}\right\}_{i \in \mathcal{I}^{\prime}}\right\rangle, \Gamma^{\prime}\right\rangle}\left(\left\langle\left\langle R^{\prime},\left\{p_{i}^{\prime}\right\}_{i \in \mathcal{I}^{\prime}}\right\rangle, \Delta\right\rangle\right)=\left\langle\left\langle\left\{p_{i}^{\prime}\right\}_{i \in \mathcal{I}^{\prime}}\right\rangle,\left\{\alpha \in\left|\operatorname{Sen}_{K}\left(\left\langle\left\{p_{i}^{\prime}\right\}_{i \in \mathcal{I}^{\prime}}\right\rangle\right)\right| \mid \rho_{\left\langle\left\{p_{i}^{\prime}\right\}_{i \in \mathcal{I}^{\prime}}\right\rangle}^{S e n}(\alpha) \in \Delta\right\}\right\rangle
$$

holds. Thus,

$$
\begin{aligned}
& \operatorname{models}_{K}\left(\sigma^{\mathrm{op}}\right)\left(\left\langle\left\langle R^{\prime},\left\{p_{i}^{\prime}\right\}_{i \in \mathcal{I}^{\prime}}\right\rangle,\left\{\alpha \in \operatorname{Sen}_{K}\left(\left\langle\left\{p_{i}^{\prime}\right\}_{i \in \mathcal{I}^{\prime}}\right\rangle\right) \mid \rho_{\left\langle\left\{p_{i}^{\prime}\right\}_{i \in \mathcal{I}^{\prime}}\right\rangle}(\alpha) \in \Delta\right\}\right\rangle\right) \\
= & \left\langle\left\langle\left\{p_{i}\right\}_{i \in \mathcal{I}}\right\rangle,\left\{\beta \in \operatorname{Sen}_{K}\left(\left\langle\left\{p_{i}\right\}_{i \in \mathcal{I}}\right\rangle\right) \mid \operatorname{Sen}_{K}(\sigma)(\beta) \in\left\{\alpha \in \operatorname{Sen}_{K}\left(\left\langle\left\{p_{i}^{\prime}\right\}_{i \in \mathcal{I}^{\prime}}\right\rangle\right) \mid \rho_{\left\langle\left\{p_{i}^{\prime}\right\}_{i \in \mathcal{I}^{\prime}}\right\rangle}(\alpha) \in \Delta\right\}\right\}\right\rangle \\
= & \left\langle\left\langle\left\{p_{i}\right\}_{i \in \mathcal{I}}\right\rangle,\left\{\beta \in \operatorname{Sen}_{K}\left(\left\langle\left\{p_{i}\right\}_{i \in \mathcal{I}}\right\rangle\right) \mid \rho_{\left\langle\left\{p_{i}^{\prime}\right\}_{i \in \mathcal{I}^{\prime}}\right\rangle}^{\operatorname{Sen}}\left(\operatorname{Sen}_{K}(\sigma)(\beta)\right) \in \Delta\right\}\right\rangle
\end{aligned}
$$


On the other hand,

$$
\begin{aligned}
& \operatorname{models}_{F O L} \circ \rho^{T h}\left(\sigma^{\mathrm{op}}\right)\left(\left\langle\left\langle R^{\prime},\left\{p_{i}^{\prime}\right\}_{i \in \mathcal{I}^{\prime}}\right\rangle, \Delta\right\rangle\right) \\
= & \operatorname{models}_{F O L}^{\mathrm{op}}\left(\rho^{T h}(\sigma)^{\mathrm{op}}\right)\left(\left\langle\left\langle R^{\prime},\left\{p_{i}^{\prime}\right\}_{i \in \mathcal{I}^{\prime}}\right\rangle, \Delta\right\rangle\right) \\
= & \operatorname{models}_{F O L}^{\mathrm{op}}\left(\varphi^{\mathrm{op}}\right)\left(\left\langle\left\langle R^{\prime},\left\{p_{i}^{\prime}\right\}_{i \in \mathcal{I}^{\prime}}\right\rangle, \Delta\right\rangle\right) \\
= & \left\langle\left\langle R,\left\{p_{i}\right\}_{i \in \mathcal{I}}\right\rangle,\left\{\alpha \in \operatorname{Sen}_{F O L}\left(\left\langle R,\left\{p_{i}\right\}_{i \in \mathcal{I}}\right\rangle\right) \mid \operatorname{Sen}(\varphi)(\alpha) \in \Delta\right\}\right\rangle
\end{aligned}
$$

Then,

$$
\begin{aligned}
& \gamma_{\left\langle\left\langle\left\{p_{i}\right\}_{i \in \mathcal{I}}\right\rangle, \Gamma\right\rangle}\left(\left\langle\left\langle R,\left\{p_{i}\right\}_{i \in \mathcal{I}}\right\rangle,\left\{\alpha \in \operatorname{Sen}_{F O L}\left(\left\langle R,\left\{p_{i}\right\}_{i \in \mathcal{I}}\right\rangle\right) \mid \operatorname{Sen}(\varphi)(\alpha) \in \Delta\right\}\right\rangle\right) \\
= & \left\langle\left\langle R,\left\{p_{i}\right\}_{i \in \mathcal{I}}\right\rangle,\left\{\beta \in \operatorname{Sen}_{K}\left(\left\langle\left\{p_{i}\right\}_{i \in \mathcal{I}}\right\rangle\right) \mid \rho_{\left\langle\left\{p_{i}\right\}_{i \in \mathcal{I}}\right\rangle}(\beta) \in\right.\right. \\
& \left.\left.\left\{\alpha \in \operatorname{Sen}_{F O L}\left(\left\langle R,\left\{p_{i}\right\}_{i \in \mathcal{I}}\right\rangle\right) \mid \operatorname{Sen}_{F O L}(\varphi)(\alpha) \in \Delta\right\}\right\}\right\rangle \\
= & \left\langle\left\langle R,\left\{p_{i}\right\}_{i \in \mathcal{I}}\right\rangle,\left\{\beta \in \operatorname{Sen}_{K}\left(\left\langle\left\{p_{i}\right\}_{i \in \mathcal{I}}\right\rangle\right) \mid \operatorname{Sen}_{F O L}(\varphi)\left(\rho_{\left\langle\left\{p_{i}\right\}_{i \in \mathcal{I}}\right\rangle}^{S e n}(\beta)\right) \in \Delta\right\}\right\rangle
\end{aligned}
$$

Then, by definition of $\rho^{T h}, \rho^{S e n}, \operatorname{Sen}_{K}$ and $\operatorname{Sen}_{F O L}$, we can get $\rho_{\left\langle\left\{p_{i}^{\prime}\right\}_{i \in \mathcal{I}^{\prime}}\right\rangle}^{\operatorname{Sen}}\left(\operatorname{Sen}_{K}(\sigma)(\beta)\right)=$ $\operatorname{Sen}_{F O L}\left(\rho^{T h}(\sigma)\left(\rho_{\left\langle\left\{p_{i}\right\}_{i \in \mathcal{I}}\right\rangle}^{S e n}(\beta)\right)\right.$.

Finally, the following lemma proves the equivalence of the two cells shown in Definition 4.2.

Lemma A.34. Let $\left\langle\left\langle\left\{p_{i}\right\}_{i \in \mathcal{I}}\right\rangle, \Gamma\right\rangle \in\left|\mathrm{Th}{ }_{K}\right|$, then $\mu_{K\left\langle\left\langle\left\{p_{i}\right\}_{i \in \mathcal{I}}\right\rangle, \Gamma\right\rangle} \circ \gamma_{\left\langle\left\langle\left\{p_{i}\right\}_{i \in \mathcal{I}}\right\rangle, \Gamma\right\rangle}=\rho_{\rho^{\text {Sign }}\left(\left\langle\left\{p_{i}\right\}_{i \in \mathcal{I}}\right\rangle\right)}^{\text {odd }} \circ$ $\mu_{F O L} \rho^{T h}\left(\left\langle\left\langle\left\{p_{i}\right\}_{i \in \mathcal{I}}\right\rangle, \Gamma\right\rangle\right) \cdot$

\section{Proof:}

We start by proving that $\mu_{K\left\langle\left\langle\left\{p_{i}\right\}_{i \in \mathcal{I}}\right\rangle, \Gamma\right\rangle} \circ \gamma_{\left\langle\left\langle\left\{p_{i}\right\}_{i \in \mathcal{I}}\right\rangle, \Gamma\right\rangle}$ and $\rho_{\rho^{S i g n}}^{\operatorname{Mod}}\left(\left\langle\left\{p_{i}\right\}_{i \in \mathcal{I}}\right\rangle\right) \mu_{F O L} \rho^{T h}\left(\left\langle\left\langle\left\{p_{i}\right\}_{i \in \mathcal{I}}\right\rangle, \Gamma\right\rangle\right)$, are the same functors. Let $\left\langle\left\{p_{i}\right\}_{i \in \mathcal{I}}\right\rangle \in\left|\operatorname{Sign}_{K}\right|, \Gamma \subseteq \operatorname{Sen}\left(\left\langle\left\{p_{i}\right\}_{i \in \mathcal{I}}\right\rangle\right)$ and $\left\langle\left\langle R,\left\{p_{i}\right\}_{i \in \mathcal{I}}\right\rangle, \Delta\right\rangle \in$ $\mid$ models $_{F O L}\left(\rho^{T h}\left(\left\langle\left\langle\left\{p_{i}\right\}_{i \in \mathcal{I}} \Gamma\right\rangle\right)\right) \mid\right.$; then, $\gamma_{\left\langle\left\langle\left\{p_{i}\right\}_{i \in \mathcal{I}}\right\rangle, \Gamma\right\rangle}\left(\left\langle\left\langle R,\left\{p_{i}\right\}_{i \in \mathcal{I}}\right\rangle, \Delta\right\rangle\right)=$ $\left\langle\left\langle\left\{p_{i}\right\}_{i \in \mathcal{I}}\right\rangle,\left\{\alpha \in \operatorname{Sen}_{K}\left(\left\langle\left\{p_{i}\right\}_{i \in \mathcal{I}}\right\rangle\right) \mid \rho_{\left\langle\left\{p_{i}\right\}_{i \in \mathcal{I}}\right\rangle}^{S e n}(\alpha) \in \Delta\right\}\right\rangle$. Thus, we obtain that:

$$
\begin{aligned}
& \mu_{K\left\langle\left\langle\left\{p_{i}\right\}_{i \in \mathcal{I}}\right\rangle, \Gamma\right\rangle} \circ \gamma_{\left\langle\left\langle\left\{p_{i}\right\}_{i \in \mathcal{I}}\right\rangle, \Gamma\right\rangle}\left(\left\langle\left\langle R,\left\{p_{i}\right\}_{i \in \mathcal{I}}\right\rangle, \Delta\right\rangle\right)= \\
& \quad \operatorname{Mod}_{K}\left(\left\langle\left\langle\left\{p_{i}\right\}_{i \in \mathcal{I}}\right\rangle,\left\{\alpha \in \operatorname{Sen}_{K}\left(\left\langle\left\{p_{i}\right\}_{i \in \mathcal{I}}\right\rangle\right) \mid \rho_{\left\langle\left\{p_{i}\right\}_{i \in \mathcal{I}}\right\rangle}(\alpha) \in \Delta\right\}\right\rangle\right) .
\end{aligned}
$$

Then, as $\mu_{F O L} \rho^{T h}\left(\left\langle\left\langle\left\{p_{i}\right\}_{i \in \mathcal{I}}\right\rangle, \Gamma\right\rangle\right)\left(\left\langle\left\langle R,\left\{p_{i}\right\}_{i \in \mathcal{I}}\right\rangle, \Delta\right\rangle\right)=\operatorname{Mod}_{F O L}\left(\left\langle\left\langle R,\left\{p_{i}\right\}_{i \in \mathcal{I}}\right\rangle, \Delta\right\rangle\right)$, the following needs to be proved:

$$
\begin{aligned}
& \rho_{\left\langle\left\{p_{i}\right\}_{i \in \mathcal{I}}\right\rangle}^{M o d}\left(\operatorname{Mod}_{F O L}(\right.\left.\left.\left(\left\langle R,\left\{p_{i}\right\}_{i \in \mathcal{I}}\right\rangle, \Delta\right\rangle\right)\right) \\
& \operatorname{Mod}_{K}\left(\left\langle\left\langle\left\{p_{i}\right\}_{i \in \mathcal{I}}\right\rangle,\left\{\alpha \in \operatorname{Sen}_{K}\left(\left\langle\left\{p_{i}\right\}_{i \in \mathcal{I}}\right\rangle\right) \mid \rho_{\left\langle\left\{p_{i}\right\}_{i \in \mathcal{I}}\right\rangle}(\alpha) \in \Delta\right\}\right\rangle\right) .
\end{aligned}
$$

The equality holds because, on the one hand, we have the modal logic reducts of the first-order predicate logic models of the formulae in $\Delta$ and, on the other hand, we have the modal logic models of the reverse translation of the formulae in $\Delta$. Notice that by the way in which tableaux are related through $T$, the formulae in $\Delta$ can be reverse translated.

The next corollary follows from Corollary A.28, and Lemmas A.33 and A.34.

Corollary A.35. $\left\langle\rho^{S i g n}, \rho^{S e n}, \rho^{M o d}, \gamma\right\rangle$ is a comorphism of satisfiability calculi. 